



\section{Patientenwohl als ethischer Maßstab für das Krankenhaus}

STELLUNGNAHME 


\section{Herausgegeben vom Deutschen Ethikrat}

Jägerstraße 22/23 D D-10117 Berlin

Telefon: $+49 / 30 / 20370-242$ - Telefax: $+49 / 30 / 20370-252$

E-Mail: kontakt@ethikrat.org

www.ethikrat.org

(C) 2016 Deutscher Ethikrat, Berlin

Alle Rechte vorbehalten.

Eine Abdruckgenehmigung wird auf Anfrage gern erteilt.

Layout: Torsten Kulick

Umschlaggestaltung: BartosKersten Printmediendesign OHG, Hamburg

ISBN 978-3-941957-71-8 (PDF) 


\section{Inhaltsverzeichnis}

ÜBERBLICK ÜBER DAS VERSORGUNGSSYSTEM KRANKENHAUS IN DEUTSCHLAND

2.1 Struktur

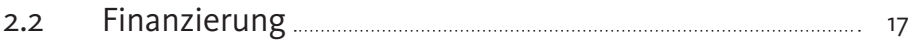

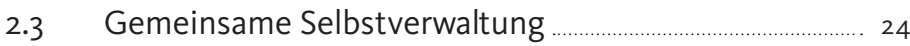

2.4 Gesetzgeberische Aktivitäten ….................................................. 28

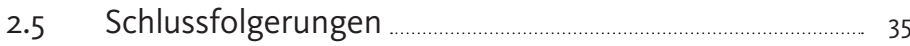

3 PATIENTENWOHL ALS ETHISCHER MASSSTAB ……..... 37

3.1 Selbstbestimmungsermöglichende Sorge ……................. 38

3.1.1 Selbstbestimmung ........................................................................................ 38

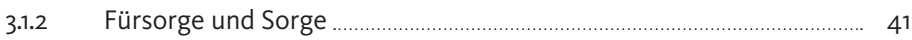

3.1.3 Arzt-Patient-Beziehung ....................................................... 42

3.1.4 Gelingende Kommunikation als Voraussetzung selbstbestimmungs-ermöglichender Sorge ............................................ 45

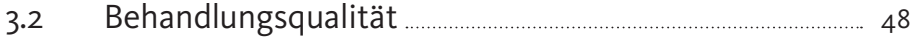

3.2.1 Qualitätsmodell und Operationalisierung ................................................ 49

3.2.2 Qualitätsmessung .............................................................................. 52

3.3 Gleicher Zugang zu Gesundheitsleistungen und gerechte Verteilung von Ressourcen ……........................... 54

3.3.1 Patienten mit besonderen Bedarfen .............................................. 57

3.3.2 Bewusster Umgang mit Ressourcen ........................................................ 58

4 GEFÄHRDUNGEN DES PATIENTENWOHLS:

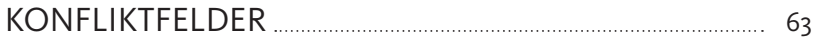

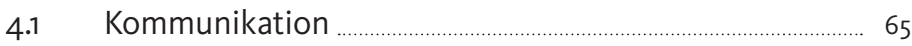

4.2 Berufsethos und Berufswirklichkeit _....................................... 67

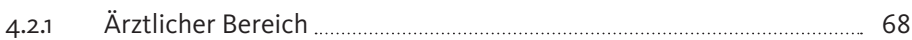

4.2.2 Pflegerischer Bereich _......................................................... 77

4.2.3 Bereich der sozialen und therapeutischen Dienste ........................... 83

4.2.4 Bereich der Krankenhausleitung ............................................. 86

4.3 Krankenhaus als Organisation ……................................... 89

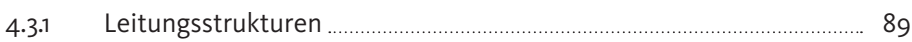

4.3.2 Klinische Ethikkomitees ………….......................................................... 91

4.4 Fort- und Weiterbildung des Krankenhauspersonals ..... 94 
4.5 Patientengruppen mit besonderen Bedarfen .................. 94

4.5.1 Kinder und Jugendliche ............................................................................ 94

4.5.2 Patienten in hohem Lebensalter _...................................... 98

4.5.3 Patienten mit geriatrietypischen Erkrankungen .................................... 100

4.5.4 Patienten mit Demenz _.......................................................... 103

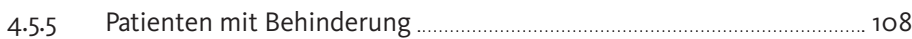

4.5.6 Patienten mit Migrationshintergrund _......................................... 111

4.5.7 Registrierte Flüchtlinge und Menschen ohne Aufenthaltsstatus ....... 113

4.6 Ressourcenallokation …............................................................ 115

4.6.1 Duale Finanzierung im stationären Sektor .................................. 117

4.6.2 Trägerstruktur im stationären Sektor ………........................................... 120

4.6.3 Anreize und Fehlanreize des DRG-Systems _............................. 122

4.7 Qualitätssicherung und Dokumentation .............................. 128

5 ZUSAMMENFASSUNG UND EMPFEHLUNGEN _........... 131

5.1 Zusammenfassung ……....................................................... 131

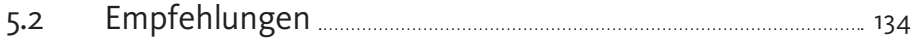

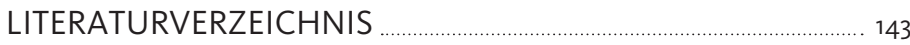

ABKÜRZUNGEN 


\section{EINLEITUNG UND PROBLEMAUFRISS}

Organisation und Finanzierung der Krankenhausversorgung in Deutschland sind Dauerthemen der politischen Diskussion. Hierzu tragen ständige Herausforderungen wie die allgemeine Kostenentwicklung im Gesundheitswesen, Weiterentwicklungen in der Medizin mit der Notwendigkeit von Investitionen und einer kontinuierlichen Anpassung der medizinischen Infrastruktur, veränderte Erwartungshaltungen aufseiten der Patienten', das Interesse, an einem volkswirtschaftlich attraktiven Wachstumsmarkt teilzunehmen, und die demografische Entwicklung in einer alternden Gesellschaft maßgeblich bei. Zwar liegt die Dichte der Krankenhäuser und die finanzielle Ausstattung des stationären Bereichs in Deutschland im internationalen Vergleich insgesamt auf einem hohen Niveau, jedoch sind durch eine vorrangige Fokussierung auf Ausgabenverringerung seitens der Krankenkassen und Ertragssteigerung auf der Seite der Anbieter Effekte entstanden, die im Hinblick auf das Patientenwohl als maßgeblicher normativer Maßstab Anlass zur Sorge geben. Zu solchen Auswirkungen können unter anderem Mengenausweitungen der Behandlungsleistungen gehören. Sie heben nicht nur den beabsichtigten Effekt der Kostensenkung auf, sondern führen, ebenso wie Reduzierungen der Behandlungsleistungen, zu Fragen nach der individuellen medizinischen Rechtfertigung. Ferner geht die Konzentration auf besonders gewinnbringende Behandlungsverfahren zulasten anderer notwendiger Behandlungsangebote. Die Arbeitsbedingungen des im Krankenhaus tätigen Personals verschlechtern sich infolge von Zeitmangel und chronischer Überlastung, sodass es zu einer sinkenden Branchenattraktivität für neue Fachkräfte und mittlerweile einem Fachkräftemangel kommt.

1 Der Einfachheit halber wird in dieser Stellungnahme die maskuline Form für beide Geschlechter verwendet. 
Solche Entwicklungen haben erhebliche Auswirkungen auf das Patientenwohl und seine normative Funktion als Leitprinzip der Gesundheitsversorgung. Zum Patientenwohl gehört nicht nur die nach medizinischen Maßstäben beurteilte körperliche und seelische Situation. Zentrale Beurteilungskriterien, ob das Patientenwohl gewahrt wird, sind darüber hinaus die Behandlungsqualität, die Selbstbestimmung des Patienten sowie die Zugangs- und Verteilungsgerechtigkeit angesichts knapper Ressourcen. Diese Kriterien markieren den ethischen Orientierungsrahmen des stationären Versorgungssystems.

Mit dem GKV-Versorgungsstärkungsgeset $z^{2}$ und dem Krankenhausstrukturgeset $z^{3}$ hat der Deutsche Bundestag im Jahr 2015 Reformen beschlossen, die verstärkt auf eine Profilierung nach Qualitätskriterien abzielen. Die Forderung des Krankenhausstrukturgesetzes, eine "qualitativ hochwertige“ und „patientengerechte“ Versorgung zu gewährleisten, stellt den Patienten und sein Wohl in den Mittelpunkt und bindet die zukünftige Ressourcenbemessung an diesen Maßstab. Eine maßgebliche Herausforderung wird allerdings darin bestehen, wie vom Gesetz gefordert, die Kriterien für eine qualitativ hochwertige und patientengerechte Versorgung näher zu bestimmen und ihre Transparenz für die Patienten und die Gesellschaft sicherzustellen.

Vor diesem Hintergrund hält es der Deutsche Ethikrat für angebracht, sich mit der Krankenhausversorgung in Deutschland aus ethischer Perspektive auseinanderzusetzen. Gute Gründe sprechen für eine Fokussierung auf das Krankenhaus, da dieses als paradigmatisch für qualitative Entwicklungen im Gesundheitssystem angesehen werden kann und verschiedene spezifische Probleme erkennen lässt:

2 Gesetz zur Stärkung der Versorgung in der gesetzlichen Krankenversicherung vom 16. Juli 2015 (BGBI. I, 1211).

3 Gesetz zur Reform der Strukturen der Krankenhausversorgung vom 10. Dezember 2015 (BGBI. I, 2229). 
》) Der Krankenhaussektor stellt mit rund 68 Milliarden Euro den größten Ausgabenblock innerhalb der Aufwendungen der gesetzlichen Krankenversicherung (insgesamt 194 Milliarden Euro) dar. ${ }^{4}$

\Krankenhausbehandlungen erlangen entgegen den bisherigen Prognosen immer größere Bedeutung. Ihre Zahl stieg in den Jahren von 2009 bis 2014 von 18,2 Millionen auf 19,5 Millionen, bei allerdings sinkender durchschnittlicher Verweildauer. ${ }^{5}$

»Im Krankenhaus treffen verschiedene Akteure mit unterschiedlichen Interessen an einem Ort aufeinander: Patienten und ihre Angehörigen, Ärzte, Pflegende und Angehörige psychosozialer und therapeutischer Berufsgruppen sowie Krankenhausmanager und Ökonomen. Hieraus erwächst ein hoher Bedarf an Kommunikations-, Organisations- und Koordinationskompetenz.

» Die Patienten im Krankenhaus sind, anders als Patienten in der ambulanten Versorgung, durch den Wechsel der Lebenssituation und den vorübergehenden Verlust der vertrauten häuslichen Lebenswelt zusätzlichen psychischen Belastungen und Einschränkungen in ihrer persönlichen Entfaltungsfreiheit ausgesetzt.

In der vorliegenden Stellungnahme werden die ethischen Probleme analysiert, die sich auf der Ebene des Krankenhauses insbesondere aus den auf der übergeordneten Allokationsebene festgelegten Rahmenbedingungen ergeben. Krankenhäuser müssen ihre Versorgungsleistungen vor dem Hintergrund politischer Vorgaben und unter ökonomischen Bedingungen erbringen, die zum Teil problematische Auswirkungen auf die Entscheidungen im ärztlichen und pflegerischen Bereich und damit auf das Patientenwohl haben. Dem Deutschen Ethikrat

4 GKV-Spitzenverband 2016, 5, 22.

5 Statistisches Bundesamt 2015a, 9. Auf der Basis der Zahlen von 2008 wurde der Wert von 19,3 Millionen Krankenhausbehandlungen für das Jahr 2030 prognostiziert (Statistische Ämter des Bundes und der Länder 2010, 11 f.). 
ist bewusst, dass er mit der Fokussierung seiner Überlegungen zur Patientenwohlorientierung auf den Krankenhaussektor nur einen Ausschnitt einer viel breiteren Problematik in den Blick genommen hat. ${ }^{6}$ So könnten grundsätzlich etwa auch die Einnahme- und Finanzierungsfragen, der Einfluss außermedizinischer Faktoren auf den Gesundheitsstatus der Menschen oder auch eine Steigerung des Patientenwohls durch eine höhere Durchlässigkeit der verschiedenen Sektoren (ambulant, stationär, rehabilitativ, Pflege, Eingliederungshilfe) stärker in den Blick genommen werden. Wenn man diese Einflussfaktoren stärker berücksichtigte, würden die hier angestellten Überlegungen zu notwendigen Maßnahmen zur Umsetzung des Patientenwohls möglicherweise eine andere Akzentuierung erfahren. Eine solch umfassende Analyse und Bewertung aber würde die Möglichkeiten dieser Stellungnahme überschreiten.

Mit dieser Stellungnahme legt der Deutsche Ethikrat Empfehlungen für eine konsequente Orientierung der Krankenhausversorgung am Patientenwohl vor.

6 In seiner Stellungnahme „Nutzen und Kosten im Gesundheitswesen - Zur normativen Funktion ihrer Bewertung" aus dem Jahr 2011 hat der Deutsche Ethikrat die Probleme bereits beschrieben, die sich auf den verschiedenen Ebenen, auf denen über die Zuteilung medizinischer Güter entschieden werden muss, für die Sicherstellung einer angemessenen Patientenversorgung ergeben. Im Mittelpunkt standen die Herausforderungen, die sich im System der Gesundheitsversorgung aus der Knappheit der zur Verfügung stehenden Ressourcen für deren gerechte Verteilung ergeben, wie dies am Beispiel der Kosten-Nutzen-Bewertung von Medikamenten untersucht wurde (Deutscher Ethikrat 2011). 


\section{2 ÜBERBLICK ÜBER DAS VERSORGUNGSSYSTEM KRANKENHAUS IN DEUTSCHLAND}

\subsection{Struktur}

Nach $₫ 107$ Abs. 1 des Fünften Buches Sozialgesetzbuch (SGB V) sind Krankenhäuser „Einrichtungen, die der Krankenhausbehandlung oder Geburtshilfe dienen, fachlichmedizinisch unter ständiger ärztlicher Leitung stehen, über ausreichende, ihrem Versorgungsauftrag entsprechende diagnostische und therapeutische Möglichkeiten verfügen und nach wissenschaftlich anerkannten Methoden arbeiten, mit Hilfe von jederzeit verfügbarem ärztlichem, Pflege-, Funktions- und medizinisch-technischem Personal darauf eingerichtet sind, vorwiegend durch ärztliche und pflegerische Hilfeleistung Krankheiten der Patienten zu erkennen, zu heilen, ihre Verschlimmerung zu verhüten, Krankheitsbeschwerden zu lindern oder Geburtshilfe zu leisten, und in denen die Patienten untergebracht und verpflegt werden können.“

Im Jahr 2014 gab es in Deutschland etwa 2.000 Krankenhäuser mit knapp 501.000 Betten. ${ }^{7}$ Davon entfielen 455.496 Betten auf 1.646 sogenannte Allgemeinkrankenhäuser, die zum einen keine reinen Tages- oder Nachtkliniken sind und sich zum anderen nicht auf Betten für psychiatrische, psychotherapeutische, neurologische und geriatrische Patienten beschränken. ${ }^{8}$ Im Vergleich mit anderen OECD-Ländern (2013) liegt die Dichte von Allgemeinkrankenhäusern (Anzahl im Verhältnis zur Bevölkerung) in Deutschland im oberen Drittel. ${ }^{9}$ Ein

7 Von den 2014 bestehenden 1.980 Krankenhäusern in Deutschland mit 500.680 Betten hatten 589 öffentliche Träger (240.195 Betten), 696 freigemeinnützige Träger (169.477 Betten) und 695 private Träger (91.008 Betten) (Statisches Bundesamt 2015b, 14).

8 Statisches Bundesamt 2015b, 15.

9 OECD.Stat (http://stats.oecd.org) unter Health: Health Care Resources: Hospitals [10.11.2015]. Langzeitpflegeeinrichtungen sind hier ausgenommen. 
entsprechender Vergleich der Krankenhausbettendichte zeigt sogar, dass Deutschland über eine der höchsten Kapazitäten an Krankenhausbetten verfügt. ${ }^{\circ}$

Die Krankenhausbehandlung erlangt immer größere Bedeutung. Wesentliche Faktoren hierfür sind die demografische Entwicklung, insbesondere die zunehmende Lebenserwartung der Bevölkerung, aber auch die zunehmende Unterversorgung im ambulanten Bereich, insbesondere in ländlichen Gebieten. Die Zahl der Krankenhausbehandlungen stieg in den Jahren 2009 bis 2014 entgegen den Prognosen von 18,2 Millionen auf 19,5 Millionen an." Die durchschnittliche Verweildauer sank dagegen kontinuierlich. Sie lag 1994 bei 12,0 Tagen, 2009 bei 8,0 Tagen und 2014 bei 7,4 Tagen. ${ }^{12}$ Dennoch bewegt sich die Verweildauer pro Behandlungsaufenthalt im OECD-Vergleich (2013) auf hohem Niveau. ${ }^{13}$ Derartige Zahlen können allerdings immer nur vor dem Hintergrund unter anderem der Verzahnung des stationären mit dem ambulanten Versorgungssystem sowie mit Blick auf die unterschiedlichen Möglichkeiten des Zugangs zu Letzterem interpretiert werden.

Die Gesamtzahl der Krankenhäuser in Deutschland hat in den letzten beiden Jahrzehnten beständig abgenommen. ${ }^{14}$ Dabei haben sich die Anteile der öffentlich-rechtlichen ${ }^{15}$ und

10 OECD.Stat (http://stats.oecd.org) unter Health: Health Care Resources: Hospital beds [10.11.2015]. Für eine detailliertere Analyse des stationären Sektors im internationalen Vergleich siehe Warschke/Lauerer/Nagels 2015.

11 Statistisches Bundesamt 2015a, 9. Auf der Basis der Zahlen von 2008 wurde der Wert von 19,3 Millionen Krankenhausbehandlungen für das Jahr 2030 prognostiziert (Statistische Ämter des Bundes und der Länder 2010, 11 f.).

12 Statistisches Bundesamt 2015b, 11.

13 OECD.Stat (http://stats.oecd.org) unter Health : Health Care Utilisation: Hospital aggregates (Variable: Curative care average length of stay) [10.11.2015]. Die Zahlen beziehen sich auf die kurative Versorgung (ohne Rehabilitation, Langzeit- und Palliativversorgung).

141991 gab es 2.411 Krankenhäuser mit 665.565 Betten, während 2014 nur noch 1.980 Krankenhäuser mit 500.680 Betten erfasst waren (Statistisches Bundesamt 2015b, 14). Zu beachten ist hierbei allerdings, dass durch die Zusammenlegung ehemals eigenständiger Krankenhäuser unter eine Institutionskennzeichnung weniger Krankenhäuser als Standorte ausgewiesen sind.

15 Öffentlich-rechtliche Krankenhausträger sind zum Beispiel Bund, Länder, Kreise, Städte und Gemeinden. 
freigemeinnützigen ${ }^{16}$ kontinuierlich zugunsten der privaten Trägerschaften ${ }^{17}$ verringert. Waren 1991 noch 46,0 Prozent der Krankenhäuser in öffentlich-rechtlicher, 39,1 Prozent in freigemeinnütziger und 14,8 Prozent in privater Trägerschaft, so waren 2014 nur noch 29,7 Prozent der Krankenhäuser in öffentlich-rechtlicher, 35,2 Prozent in freigemeinnütziger und bereits 35,1 Prozent in privater Hand..$^{8}$

Dennoch stand 2014 knapp die Hälfte aller Betten in den vergleichsweise großen öffentlich-rechtlichen Krankenhäusern (48,0 Prozent), während freigemeinnützige Häuser 33,8 Prozent und private Krankenhäuser 18,2 Prozent der verfügbaren Betten stellten. ${ }^{19}$ In einer ökonomischen Perspektive zeigt sich ein wesentlicher Unterschied zwischen privaten Krankenhausträgern einerseits und öffentlichen und freigemeinnützigen Trägern andererseits in der Zweckbindung: „Primäreffekt der betrieblichen Betätigung [...] ist die Gewinnerzielung, Sekundäreffekt dagegen die Bedarfsdeckung. "20 Dies ist Ausdruck der grundrechtlich geschützten Berufs- und Wettbewerbsfreiheit der Träger privater Krankenhäuser. ${ }^{21}$ Die Teilnahme von Krankenhäusern in privater Trägerschaft an der Allgemeinkrankenhausversorgung setzt gemäß $\$ 5$ Abs. 1 Nr. 2 des Krankenhausfinanzierungsgesetzes ${ }^{22}$ voraus, dass diese gemeinnützig im Sinne von $\$ 67$ der Abgabenordnung sind. Solche Krankenhäuser werden öffentlich gefördert, wenn bei ihnen mindestens 40 Prozent der jährlichen Belegungstage oder Berechnungstage auf Patienten entfallen, bei denen nur Entgelte

16 Freigemeinnützige Krankenhausträger sind zum Beispiel Kirchen, Wohlfahrtsverbände und private Stiftungen.

17 Nach $\S 30$ der Gewerbeordnung vom 22. Februar 1999 (BGBI. I, 202), zuletzt geändert durch Art. 10 des Gesetzes vom 11. März 2016 (BGBI. I, 396): konzessionierte Erwerbsunternehmen.

18 Statistisches Bundesamt 2015b, 8.

19 Ebd.

20 So Eichhorn 1975, 24.

21 Wernick, in: Huster/Kaltenborn 2010, $16 \mathrm{C} \mathrm{Rn.} \mathrm{8.} \mathrm{ff.}$

22 Gesetz zur wirtschaftlichen Sicherung der Krankenhäuser und zur Regelung der Krankenhauspflegesätze vom 29. Juni 1972 (BGBI. I, 1009), neugefasst durch Bekanntmachung vom 10. April 1991 (BGBI. I, 886), zuletzt geändert durch Art. 1 des Gesetzes vom 10. Dezember 2015 (BGBI. I, 2229). 
für allgemeine Krankenhausleistungen (siehe $\$ 7$ Krankenhausentgeltgesetz ${ }^{23}, \$ 10$ Bundespflegesatzverordnung ${ }^{24}$ ) berechnet werden, also nicht auch Wahlleistungen vereinbart wurden. ${ }^{25}$ Öffentlich geförderte Krankenhäuser in privater Trägerschaft müssen sich also in entsprechendem Umfang an der allgemeinen Versorgung gesetzlich versicherter Patienten beteiligen.

Die Bettenauslastung lag 2014 bei 77,4 Prozent, wobei es zwischen den genannten Formen der Trägerschaften diesbezüglich kleinere Unterschiede gibt. Die durchschnittliche Bettenauslastung lag in den öffentlich-rechtlichen Krankenhäusern 2014 bei 79,4 Prozent, bei den freigemeinnützigen bei 75,7 Prozent und bei den privaten bei 75,6 Prozent. ${ }^{26}$ Größere Differenzen bestehen zwischen verschiedenen Fachabteilungen. ${ }^{27}$

Die Landeskrankenhausplanung der Bundesländer soll eine bedarfsgerechte Versorgung sicherstellen. In den Versorgungsaufträgen werden die Fachabteilungen und Bettenzahlen festgelegt. Diese Planung des Versorgungsrahmens und der stationären Kapazitäten obliegt den Bundesländern und berücksichtigt unterschiedliche Versorgungsstufen, die nicht einheitlich definiert sind. Grob lässt sich folgende Einteilung vornehmen: Krankenhäuser der Grundversorgung verfügen mindestens über eine Abteilung der inneren Medizin oder der Chirurgie, ohne dabei separate Abteilungen für

23 Gesetz über die Entgelte für voll- und teilstationäre Krankenhausleistungen vom 23. April 2002 (BGBI. I, 1412, 1422), zuletzt geändert durch Art. 4 des Gesetzes vom 10. Dezember 2015 (BGBI. I, 2229).

24 Verordnung zur Regelung der Krankenhauspflegesätze vom 26. September 1994 (BGBI. I, 2750), zuletzt geändert durch Art. 5 des Gesetzes vom 10. Dezember 2015 (BGBI. I, 2229).

25 Siehe dazu Genzel/Degener-Hencke, in: Laufs/Kern 2010, §81 Rn. 32.

26 Statistisches Bundesamt 2015b, 20.

27 Unabhängig vom Träger weisen Fachabteilungen wie innere Medizin (79,9 Prozent), Geriatrie (91,3 Prozent) sowie Psychiatrie und Psychotherapie (93,5 Prozent) höhere durchschnittliche Bettenauslastungen auf, Fachabteilungen wie Augenheilkunde (64,o Prozent), Hals-Nasen-OhrenHeilkunde (61,1 Prozent) oder Kinderheilkunde (66,3 Prozent) niedrigere Auslastungen (Statistisches Bundesamt 2015b, 25). 
Teilbereiche einer Fachrichtung vorzuhalten. Letztgenanntes gilt auch für Häuser der Regelversorgung, die aber neben der inneren Medizin und Chirurgie darüber hinaus auch beispielsweise Abteilungen für Gynäkologie und Geburtshilfe, HalsNasen-Ohren-Heilkunde oder Pädiatrie vorhalten. Häuser, die zudem mindestens separate Abteilungen für Unfall- und Viszeralchirurgie, Radiologie und Anästhesie vorhalten sowie weitere Fachrichtungen abdecken, werden der Schwerpunkt- bzw. Zentralversorgung zugeordnet. Noch weiter differenzierte und entsprechend ausgestattete Häuser werden der Maximalversorgung zugerechnet. ${ }^{28}$ Die klare Zuordnung einzelner, insbesondere kleinerer Häuser zu einer der genannten Versorgungsstufen ist zunehmend schwierig, da viele Häuser im Rahmen allgemeiner Fachabteilungen sogenannte Departments betreiben, die eine Spezialisierung innerhalb des jeweiligen Faches aufweisen. Die verschiedenen Versorgungsstufen korrespondieren mit lokalen, regionalen und überregionalen Einzugsbereichen.

Generell lässt sich die innerbetriebliche Organisation eines Krankenhauses gliedern in die jeweils hierarchisch geordneten Bereiche des Ärztlichen Dienstes, des Pflegepersonals, der im Krankenhaus tätigen Gesundheitsberufe (wie Hebammen, Physiotherapeuten, Ergotherapeuten, Kunst- und Gestaltungstherapeuten, Psychotherapeuten, Heilpädagogen, Logopäden) und Sozialberufe (wie Sozialarbeiter und Seelsorger) sowie die auch die wirtschaftliche Steuerung umfassende Verwaltung. ${ }^{29}$ Die Leitung obliegt einer vom Träger bestimmten Geschäftsführung - entweder einem meist kaufmännischen Geschäftsführer oder Leiter oder einem Krankenhausdirektorium (meist ärztlicher Direktor, Verwaltungsdirektor und Pflegedienstleitung). ${ }^{30}$ Zum Jahresende 2014 waren in Deutschland 1,2 Millionen Menschen in Krankenhäusern

28 Vgl. Nagel 2012, 27 f.; Nagel 2013, $154 \mathrm{f}$.

29 Vgl. Nagel 2013, $151 \mathrm{f}$.

$30 \mathrm{Vgl}$. ebd. 
beschäftigt - 14,4 Prozent davon im Ärztlichen Dienst. ${ }^{31}$ Im OECD-Ländervergleich liegt die Anzahl der Beschäftigten in deutschen Krankenhäusern im Verhältnis zur Bevölkerung (2013) etwas über dem Durchschnitt. ${ }^{32}$

Dabei entwickelte sich laut Statistischem Bundesamt die Zahl der Vollstellen im ärztlichen Bereich von 110.152 im Jahr 2001 auf 150.757 im Jahr 2014, im Bereich der Pflege von $331.472 \mathrm{im}$ Jahr 2001 auf $318.749 \mathrm{im}$ Jahr 2014. ${ }^{33}$ Die Pflegekräfte setzen sich zusammen aus examinierten Gesundheits- und Krankenpflegekräften (81 Prozent), examinierten Gesundheits- und Kinderpflegekräften ${ }^{34}$ (9 Prozent) und Pflegehelfern (4 Prozent). Etwa 6 Prozent sind sonstige Pflegepersonen, wie beispielsweise Altenpflegekräfte. ${ }^{35}$ Eine große Zahl von bundesdeutschen Kliniken bedient sich insbesondere im Falle von Personalengpässen bei der Besetzung der Stellen im pflegerischen und im therapeutischen Bereich in wachsendem Maße sogenannter „Leasingkräfte“ oder hat einen Teil des Personals in Tochtergesellschaften der Krankenhäuser oder in Beschäftigungsgesellschaften ausgegliedert. Die Kosten für diese Mitarbeiter erscheinen dann nicht mehr auf der Liste der jeweiligen Vollkräfte einer Berufsgruppe eines Hauses, sondern werden unter dem Sachkostenbudget geführt. Die Gesamtanzahl der in einem Personal-Leasingverfahren im Krankenhaus

31 Statistisches Bundesamt 2015b, 8.

32 OECD.Stat (http://stats.oecd.org) unter Health: Health Care Resources: Total health and social employment [10.11.2015].

33 Zum ärztlichen Bereich: Statistisches Bundesamt 2015b, 12; zum Bereich der Pflege: Statistisches Bundesamt 2008, 17; Statistisches Bundesamt 2015b, 26. Als Vollkräfte erfasst das Statistische Bundesamt hier die Zahl der auf die volle tarifliche Arbeitszeit umgerechneten Beschäftigten im Jahresdurchschnitt mit einem direkten Beschäftigungsverhältnis bei der Einrichtung, also ohne Personal-Vollstellen, die im Leasingverfahren bewirtschaftet werden.

34 Die Zahl der im Krankenhaus arbeitenden Gesundheits- und Kinderkrankenpflegekräfte nimmt beständig ab, von 40.650 im Jahr 2002 auf 36.900 im Jahr 2011 (Berufsgenossenschaft für Gesundheitsdienst und Wohlfahrtspflege 2013, 31).

35 Berufsgenossenschaft für Gesundheitsdienst und Wohlfahrtspflege 2013, 30. 
eingesetzten Vollstellen ${ }^{36}$ ist von 14.009 im Jahr 2009 auf 22.545 im Jahr 2014 angestiegen, davon entfielen auf den ärztlichen Bereich 3.052 und auf die Pflege 6.487 Vollstellen. ${ }^{37}$

Aus den dargestellten Zahlen des Statistischen Bundesamtes geht hervor, dass sich das zahlenmäßige Verhältnis von Ärzten zu Pflegekräften (unter Mitzählung der jeweiligen Leasingkräfte) stark verändert hat: Kamen 2001 auf einen Arzt drei Pflegekräfte, so waren es 2014 nur noch 2,1. ${ }^{38}$ Die Gründe hierfür sind vielschichtig. Wesentlich ist das Inkrafttreten des Arbeitszeitgesetzes am 1. Juli 1994, wodurch die im ärztlichen Bereich weitverbreitete Praxis von 24-stündigen oder noch längeren Dienstzeiten unterbunden und für die Sicherstellung der ärztlichen Patientenversorgung im Krankenhaus eine Anpassung der Personalschlüssel notwendig wurde.

\subsection{Finanzierung}

Die sogenannte duale Finanzierung der Krankenhäuser erfolgt seit 1972 auf der Grundlage des Krankenhausfinanzierungsgesetzes (KHG). Dieses gilt für alle Krankenhäuser, die nach $\$ 108$ SGB V zur Behandlung sowohl von Versicherten der gesetzlichen Krankenversicherung als auch von solchen der privaten Krankenversicherung (sogenannten Selbstzahlern) zugelassen sind. In Umsetzung der sozialstaatlichen Gewährleistungsverantwortung bezieht das Krankenhausfinanzierungsgesetz den weitaus größten Teil der Krankenhäuser in ein staatliches Planungssystem mit öffentlicher Förderung

36 Bei der Zählung der Vollstellen, die im Personal-Leasingverfahren bewirtschaftet werden, ist entscheidend, dass sich das Krankenhaus für seine ansonsten in der Einrichtung selbst erbrachten Leistungen Personalverstärkung in Form von Zeitarbeit o. Ä. hinzuholt. Personal einer Fremdfirma, die zum Beispiel die Reinigung in der Einrichtung übernommen hat, wird damit nicht erfasst.

37 Statistisches Bundesamt 2015b, 12, 33.

38 Verhältnisse basieren auf Zahlen für den ärztlichen Bereich (Statistisches Bundesamt 2015b, 12) und den Bereich der Pflege (Statistisches Bundesamt 2008, 17; Statistisches Bundesamt 2015b, 26). 
der Investitionskosten und gesetzlich vorgegebener Finanzierung der notwendigen Betriebskosten ein. Unmittelbarer Zweck des Krankenhausfinanzierungsgesetzes ist gemäß dem seit 1972 unverändert gebliebenen $₫ 1$ Abs. 1 KHG die wirtschaftliche Sicherung der Krankenhäuser. Allerdings ist damit kein Selbstzweck formuliert; die wirtschaftliche Sicherung der Krankenhäuser dient vielmehr als Mittel zum Zweck einer bedarfsgerechten Krankenversorgung zu sozial tragbaren Krankenhauskosten. ${ }^{39}$ Die Umsetzung dieser Grundkonzeption ist geprägt vom Grundsatz der Trägervielfalt. Sie wird durch $\$ 1$ Abs. 2 Satz 1 KHG vorgegeben. $\$ 1$ Abs. 2 Satz 2 modifiziert diesen Grundsatz dahingehend, dass nach Maßgabe des Landesrechts insbesondere die wirtschaftliche Sicherung freigemeinnütziger und privater Krankenhäuser zu gewährleisten ist. ${ }^{40}$ Private Krankenhäuser werden dabei in der Regel nach erwerbswirtschaftlichen Grundsätzen betrieben. ${ }^{41}$ Zur Verwirklichung der Ziele des Gesetzes, die Krankenhäuser wirtschaftlich abzusichern, „um eine qualitativ hochwertige, patienten- und bedarfsgerechte Versorgung der Bevölkerung mit leistungsfähigen, qualitativ hochwertig und eigenverantwortlich wirtschaftenden Krankenhäusern zu gewährleisten und zu sozial tragbaren Pflegesätzen beizutragen“ ( $\$ 1$ Abs. $1 \mathrm{KHG})$, müssen die Bundesländer Landeskrankenhauspläne aufstellen. Einzelheiten regelt das Landesrecht. ${ }^{42}$

Die wirtschaftliche Sicherung der Krankenhäuser erfolgt demnach aus zwei Quellen: Zum einen haben Häuser, die in die Krankenhauspläne der Länder aufgenommen wurden, einen Anspruch auf Förderung von Investitionen durch das

39 Siehe zum Letzteren BVerfGE 82, 209 (225); im Übrigen zur Grundkonzeption etwa Quaas, in: Quaas/Zuck 2014, §26 Rn. 3-5 m. w. N.

40 Zur Privilegierung der freigemeinnützigen und privaten Träger, die im Gegensatz zu Krankenhäusern in öffentlicher Trägerschaft, insbesondere zu kommunalen Krankenhäusern, in aller Regel nicht auf zusätzliche Betriebsund Investitionszuschüsse ihrer Träger zurückgreifen können, vgl. Deutscher Bundestag 1984, 27; ferner Quaas, in: Quaas/Zuck 2014, § 25 Rn. $77 \mathrm{ff}$.

41 Der Unternehmer einer solchen „Privatkrankenanstalt“ bedarf nach $\S 30$ der Gewerbeordnung einer gewerberechtlichen Konzession.

42 Vgl. Fleischhauer 2015, $21 \mathrm{f}$. 
jeweilige Bundesland, zum anderen werden die Betriebs- und Personalkosten von den Krankenkassen getragen. Daneben tragen Patienten mit direkten verpflichtenden oder freiwilligen Zuzahlungen (tageweise Krankenhauszuzahlung, Wahlleistungen) in gewissem Umfang zur Finanzierung des stationären Sektors bei. Die Investitionsfördersummen der einzelnen Bundesländer unterscheiden sich erheblich und sind im zeitlichen Verlauf seit vielen Jahren stetig rückläufig. Wegen der Finanzknappheit der Länder sind heute zudem Wartezeiten von bis zu zehn Jahren für einen verbindlichen Fördermittelbescheid üblich, der dann darüber hinaus oft lediglich Ratenzahlung vorsieht. Dies zwingt viele Krankenhäuser zum Aufschub wichtiger Bau- und Investitionsmaßnahmen oder $\mathrm{zu}$ alternativer Kreditfinanzierung bei nicht aufschiebbaren Auflagen, wie zum Beispiel beim Brandschutz, die zu einer existenziell bedrohlichen Finanzlage führen können. Generell wird diese Praxis der schleppenden und zu gering ausfallenden Förderung für den mittlerweile eingetretenen erheblichen Investitionsstau verantwortlich gemacht. Aktuellen Analysen zufolge liegt der jährliche Investitionsbedarf der Krankenhäuser ohne Universitätskliniken bei ca. 5,3 Milliarden Euro und damit doppelt so hoch wie die von den Ländern zur Verfügung gestellten Investitionsmittel. ${ }^{43}$

Das Krankenhausfinanzierungsreformgesetz ${ }^{44}$ von 2009 sah ab 2012 Investitionspauschalen statt jeweils verhandelter und politisch entschiedener Investitionsfinanzierungen vor ( $\$ 10$ Abs. $1 \mathrm{KHG}$ ), was aber bisher uneinheitlich umgesetzt wurde. $\mathrm{Zu}$ unterscheiden ist zwischen der Einzel- und der Pauschalförderung: Über die Pauschalförderung werden die laufenden Investitionskosten der Häuser abgedeckt ${ }^{45}$, während für größere Anschaffungen und die Einrichtung oder

43 Vgl. Augurzky et al. 2015, 175.

44 Gesetz zum ordnungspolitischen Rahmen der Krankenhausfinanzierung ab dem Jahr 2009 vom 17. März 2009 (BGBI. I, 534).

45 Für die Hochschulkliniken gelten besondere Finanzierungsregelungen. 
Sanierung ganzer Abteilungen Mittel im Rahmen der Einzelförderung zur Verfügung gestellt werden können.

Die Betriebskosten (Sach- und Personalkosten) werden durch die Leistungsentgelte getragen $(\$ 17 \mathrm{KHG})$. Bis 2003 galt das Kostendeckungsprinzip: Die laufenden, tatsächlich entstandenen Kosten wurden übernommen, indem für jeden Tag eines Patienten im Krankenhaus ein tagesgleicher Pflegesatz in Rechnung gestellt wurde. ${ }^{46}$ Seit 2004 erfolgt die Vergütung der allgemeinen Krankenhausleistungen nicht mehr durch die Abrechnung der Pflegesätze, sondern diese werden in diagnosebezogenen Fallpauschalen (diagnosis related groups, DRGs; siehe unten) erfasst, die alle Leistungen für einen Behandlungsfall einschließen ( $\$ 17 \mathrm{~b} \mathrm{KHG}$ ); von den DRGs ausgenommen sind die in psychiatrischen und psychosomatischen Einrichtungen erbrachten Leistungen, die weiterhin nach Pflegesätzen abgerechnet werden und für die nach entsprechenden Einsprüchen der Bundesärztekammer und der Fachverbände gegen die Einführung eines DRG-ähnlichen Finanzierungssystems ein neues Entgeltsystem eingeführt werden soll, bei dem regionale Bedingungen und hausindividuelle Besonderheiten in eine fallgruppenbasierte Budgetierung eingehen sollen. ${ }^{47}$

Für andere „besondere Einrichtungen“ gemäß $₫ 17 \mathrm{~b}$ Abs. 1 Satz 10 KHG, wie beispielsweise Palliativstationen, besteht schon heute die Möglichkeit, krankenhausindividuelle

46 Der Pflegesatz ist gemäß $§ 2$ KHG das Entgelt, das der Patient oder sein Kostenträger für die stationären oder teilstationären Leistungen des Krankenhauses zu entrichten hat, und besteht aus dem Entgelt für die allgemeinen Krankenhausleistungen und weiteren Kosten des Krankenhauses, sofern diese pflegesatzfähig sind.

47 In dem Papier „Eckpunkte zur Weiterentwicklung des Psych-Entgeltsystems" des Bundesministeriums für Gesundheit vom 18. Februar 2016 wird zwar am Grundsatz der Leistungsorientierung und empirischen Kalkulation festgehalten, strukturelle Besonderheiten der einzelnen Krankenhäuser, wie beispielsweise die Schwerpunktsetzung auf bestimmte Diagnosegruppen, sollen aber ebenso berücksichtigt werden wie die jeweilige Versorgungsstufe, denen die Krankenhäuser zugeordnet sind. Grundlage soll zunächst die derzeit gültige Psychiatrie-Personalverordnung sein. Der G-BA soll aber nach einer Übergangsfrist neue Mindestvorgaben für die zukünftige Personalausstattung machen. Das Hometreatment durch die Kliniken soll erleichtert werden. 
Entgeltsätze zu vereinbaren. Die Vergütung für Wahlleistungen (zum Beispiel Unterbringung im Einzelzimmer, Chefarztbehandlung etc.) wird wie bei dem vormaligen System der tagesgleichen Pflegesätze als Zuschlag, den der Patient selbst bezahlen muss, gesondert berechnet.

Die Entwicklung des DRG-Systems, seine jährliche Anpassung an veränderte Verhältnisse, wie zum Beispiel medizinische Entwicklungen, Kostenentwicklungen etc., sowie weitere Aufgaben wurden durch das Krankenhausfinanzierungsgesetz an die gemeinsame Selbstverwaltung (siehe 2.3) delegiert und erfolgen durch Vereinbarungen, die vom GKV-Spitzenverband und dem Verband der Privaten Krankenversicherung gemeinsam mit der Deutschen Krankenhausgesellschaft getroffen werden. Diese Vertragspartner können mit den zur Erfüllung dieser Vorschriften erforderlichen Arbeiten ein eigenes „DRG-Institut“ beauftragen. Mit dieser Aufgabe wurde das Institut für das Entgeltsystem im Krankenhaus (InEK) betraut (siehe 2.3)..$^{8}$

In DRGs werden Diagnosen deutschlandweit anhand unterschiedlicher Kriterien (darunter die Haupt- und Nebendiagnose sowie Prozeduren) in medizinisch und (im Hinblick auf den dafür erforderlichen Aufwand) ökonomisch möglichst homogene Fallgruppen klassifiziert. Das pauschalierende Verfahren arbeitet mit einem Patientenklassifikationssystem (G-DRG-System), das auf den deutschen Versionen des internationalen Diagnoseschlüssels (ICD-10-GM) mit etwa 15.000 Positionen und einem Operationen- und Prozedurenschlüssel (OPS) mit etwa 25.000 Positionen basiert. Beide Schlüsselkataloge werden vom Deutschen Institut für Medizinische Dokumentation und Information veröffentlicht und beständig fortgeschrieben. Die Vergütung für jeden einzelnen Behandlungsfall stützt sich auf die nach dem ICD-Schlüssel kodierte Hauptdiagnose und eventuelle Nebendiagnosen sowie die für diesen Fall nach dem OPS-Schlüssel kodierten erbrachten

48 Vgl. Fleischhauer 2015, $22 \mathrm{f}$. 
Leistungen. Mithilfe eines vom InEK zertifizierten Algorithmus, des sogenannten Groupers, verarbeitet das InEK die kodierten Daten und ermittelt die Fallgruppen. Nach Zuordnung des Behandlungsfalls zu einer Fallgruppe kann die zutreffende Fallpauschale nach einem ebenfalls vom InEK veröffentlichten Fallpauschalenkatalog ermittelt werden. ${ }^{49}$ Das dem Krankenhaus für die betreffende Fallpauschale zustehende Entgelt errechnet sich aus der Multiplikation der sogenannten Bewertungsrelation, die das Kostengewicht einer Fallgruppe in Relation zum durchschnittlichen Kostengewicht aller im Krankenhaus behandelten Fälle ausdrückt, mit dem Basisfallwert, der den Vergütungsbetrag für einen Fall mit der Bewertungsrelation 1,0 beschreibt. Nach Vereinbarung von individuellen Basisfallwerten zunächst für jedes Krankenhaus wurden ab 2005 länderspezifische Werte berechnet. Bis 2009 erfolgte eine gestufte Anpassung (Konvergenzphase) an das landesspezifische Preisniveau. Seit 2014 gilt ein bundeseinheitlicher Basisfallwert als Orientierungsgröße mit einem Korridor, auf den sich die Landesbasisfallwerte schrittweise zubewegen $(\$ 10$ Abs. 8 Krankenhausentgeltgesetz). Bei der Kalkulation der Vergütung sind neben der Fallpauschale bestimmte, im Krankenhausfinanzierungsgesetz aufgeführte Zusatzentgelte und $\mathrm{Zu}$ - bzw. Abschläge zu berücksichtigen. Es ist offensichtlich, dass die Abrechnung nach Fallpauschalen zeitaufwendig ist und spezielle Kenntnisse erfordert. Fehler können erhebliche finanzielle Auswirkungen haben. Viele Krankenhäuser haben für die Abrechnung nach diesem System zusätzliches Personal eingestellt bzw. einstellen müssen. ${ }^{50}$

Die künftige Leistungsmenge eines Krankenhauses wird zwischen dem Träger des Hauses und den Krankenkassen unter Berücksichtigung des Umfangs der erbrachten Leistungen und erwarteter Leistungsveränderungen jährlich im Voraus als sogenanntes Erlösbudget verhandelt und vereinbart. 
Dabei werden die seitens der Krankenkassen vom Krankenhaus geforderten DRGs mit dem Versorgungsauftrag verglichen. Verschiedenen DRGs werden verschiedene Gewichte zugemessen (Bewertungsrelationen). Aus der Gesamtheit der in einem individuellen Krankenhaus jährlich geleisteten DRGs kann durch Summierung ihrer Bewertungsrelationen der sogenannte Case-Mix berechnet werden. Das jährliche Budget des Krankenhauses errechnet sich dann aus der Multiplikation des Case-Mix mit dem Basisfallwert. Abweichungen von den vorab vereinbarten Leistungsmengen führen zu Mehr- bzw. Mindererlösen. Diese werden im Folgejahr in einem Erlösausgleich berücksichtigt..51 Um unbegründete Leistungsausweitungen zu verhindern, müssen die Krankenhäuser auf der Grundlage der Änderung des Krankenhausentgeltgesetzes von 2011 einen Mehrleistungsabschlag52 zahlen. Umgekehrt wird den steigenden Kosten, die in vielen Häusern zu finanziellen Krisensituationen geführt haben, durch flankierende Gesetzesmaßnahmen, wie dem Krankenhaus-Finanzhilfepaket 2013/2014 und dem Krankenhausstrukturgesetz von 2015, mit Versorgungszuschlägen, Tarifausgleichsraten und Sicherstellungszuschlägen begegnet.

Die leistungsorientierte Vergütung nach DRG bezieht sich somit nicht auf die tatsächlichen Kosten einer Krankenhausbehandlung und soll zu einem starken Anreiz für wirtschaftliches Handeln führen. Gewinn und Verlust hängen davon ab, ob es gelingt, effizienter zu wirtschaften als bei der Kalkulation der Pauschalvergütung berechnet. Das Ziel einer wirtschaftlichen Betriebsführung von Krankenhäusern ist bereits aus Gerechtigkeitserwägungen zu bejahen. Jedoch sind bei jeder

51 Vgl. Nagel 2012, $51 \mathrm{f}$.

52 Nach dem neuen Krankenhausstrukturgesetz heißt dieser Fixkostendegressionsabschlag (Art. 2 Nr. 10h). Verhandelt zum Beispiel ein fiktives Krankenhaus anstelle der bisher vereinbarten 20.000 Case-Mix-Punkte nun 21.000 Punkte mit den Kassen, werden die zusätzlichen 1.00o Punkte mit einem prozentualen Abschlag über mehrere Jahre belegt. Sinn dieser Regelung ist es, Mengenausweitungen im Krankenhauswesen zu erschweren. Gleichzeitig muss damit aber auch das Wachstum vorfinanziert werden. 
Form von Vergütung mögliche Fehlanreize zu bedenken (vgl. Kapitel 4).

\subsection{Gemeinsame Selbstverwaltung}

Die Gesundheitsversorgung insgesamt und auch die stationäre Versorgung in Deutschland ist vom Gesetzgeber weitgehend der sogenannten gemeinsamen Selbstverwaltung der Kassen sowie der Spitzenorganisationen der in der GKV-Versorgung tätigen Ärzte und Krankenhäuser anvertraut. Vor allem der von ihnen getragene Gemeinsame Bundesausschuss (G-BA) setzt untergesetzliches Recht, das für Kassen, Leistungserbringer wie Krankenhäuser und Versicherte unmittelbar verbindlich ist. Die wichtigsten Elemente dieses Systems der gemeinsamen Selbstverwaltung seien im Folgenden skizziert.

Die derzeit 132 gesetzlichen Krankenkassen (Ortskrankenkassen, Betriebskrankenkassen, Innungskrankenkassen, Ersatzkassen) bilden die gesetzliche Krankenversicherung, bei der insgesamt ca. 90 Prozent der Bevölkerung versichert sind. ${ }^{53}$ Die gesetzliche Krankenversicherung ist eine Pflichtversicherung für Arbeiter und Angestellte, deren Jahresverdienst eine bestimmte Grenze, die Versicherungspflichtgrenze oder Jahresentgeltgrenze, unterschreitet. Sie arbeitet nach dem Solidarprinzip, das heißt, die Beitragshöhe ist vom Einkommen und nicht vom Risiko und die Versicherungsleistung nicht von der Beitragshöhe abhängig. Die gesetzlichen Krankenkassen bilden in jedem Bundesland einen Landesverband und werden vom gesetzlich vorgeschriebenen Spitzenverband Bund der Krankenkassen (GKV-Spitzenverband) unterstützt. Letzterer ist in allen Gremien vertreten, die auf Bundesebene über untergesetzliche Normen für die medizinische und zahnärztliche Versorgung der Versicherten Entscheidungen

53 Die folgenden Darstellungen in Abschnitt 2.3 beruhen im Wesentlichen auf Fleischhauer 2015. 
treffen, und besitzt daher erheblichen politischen Einfluss. Das SGB V schreibt vor, dass die Landesverbände der verschiedenen Krankenkassen in jedem Bundesland gemeinsam eine Arbeitsgemeinschaft „Medizinischer Dienst der Krankenversicherung" bilden, der unter anderem die Notwendigkeit von Leistungen zulasten der gesetzlichen Krankenversicherung überprüft ( $\$ 278$ SGB V). ${ }^{54}$

Die privaten Krankenversicherungen, bei denen im Jahr 2013 in Deutschland ca. 10 Prozent der Bevölkerung versichert waren, werden im Gegensatz zur gesetzlichen Krankenversicherung nicht durch einkommensbezogene Mitgliedsbeiträge, sondern durch Prämien finanziert, die nach dem Prinzip der individuellen Risikoabschätzung berechnet werden. Im Unterschied zur gesetzlichen Krankenversicherung gilt bei der privaten Krankenversicherung für den Arzt nur der Behandlungsvertrag mit dem Patienten, das heißt, im Gegensatz zum Vertragsarzt hat der Arzt keinerlei vertragliche Verpflichtungen gegenüber der Krankenversicherung und rechnet seine Leistungen direkt mit dem Patienten ab. Auf Landes- und Bundesebene werden die Interessen der privaten Krankenversicherungen durch den Verband der Privaten Krankenversicherung wahrgenommen, dem 43 Unternehmen angehören.

Die gesetzlichen Krankenkassen dürfen Krankenhausbehandlungen ihrer Versicherten nur erbringen lassen, wenn das Krankenhaus entweder als Hochschulklinik anerkannt ist, wenn es in den Krankenhausplan eines Bundeslandes aufgenommen ist oder wenn es mit einem Landesverband der Krankenkassen einen Versorgungsvertrag abgeschlossen hat ( $\$ 108$ SGB V). Die Krankenhausplanung obliegt den Bundesländern, die jeweils einen Landeskrankenhausplan und ein Investitionsprogramm aufstellen müssen. Nur Krankenhäuser, die in den Landeskrankenhausplan aufgenommen sind, können finanzielle Förderung aus dem staatlichen Investitionsprogramm

$54 \mathrm{Zu}$ den Aufgaben des Medizinischen Dienstes der Krankenversicherung siehe $§ 275$ SGB V. 
erhalten. Der Versorgungsvertrag verpflichtet die Landesverbände der Krankenkassen, mit dem Krankenhausträger unter Beachtung des SGB V bei Häusern, die nach DRGs abrechnen, Budgetverhandlungen über Art und Menge der Leistungen, bei Häusern außerhalb des DRG-Systems wie beispielsweise psychiatrischen Krankenhäusern Pflegesatzverhandlungen zu führen. Die vereinbarten Leistungsarten und Mengen bzw. die Pflegesätze gelten jeweils für alle Patienten des betreffenden Krankenhauses, somit für Versicherte der gesetzlichen Krankenversicherung als auch für privat versicherte Patienten. Durch den Zusammenschluss von Trägern von Krankenhäusern, die in einem Bundesland zugelassen sind, werden die Landeskrankenhausgesellschaften gebildet, die gesetzlich übertragene Aufgaben in den einschlägigen Ausschüssen der Selbstverwaltung ausführen. Die Landeskrankenhausgesellschaften wiederum bilden die Deutsche Krankenhausgesellschaft, die den Spitzenverband der Krankenhäuser auf Bundesebene darstellt und unter anderem vom Gesetzgeber übertragene Aufgaben in der Selbstverwaltung wahrnimmt.

Die Ärzte sind nach den Heilberufsgesetzen zur Mitgliedschaft in der Landesärztekammer in dem betreffenden Bundesland verpflichtet. Die Ärztekammern der Länder haben sich auf Bundesebene zur Bundesärztekammer zusammengeschlossen. In jedem Bundesland gibt es zudem eine Kassenärztliche und eine Kassenzahnärztliche Vereinigung. Ihnen gehören durch Gesetz alle zur vertragsärztlichen Tätigkeit zugelassenen Ärzte bzw. Zahnärzte an. Sie wirken mit den Krankenkassen zusammen, um die vertragsärztliche ambulante Versorgung der Versicherten sicherzustellen, verteilen die Gesamtvergütung und nehmen wichtige Aufgaben in der Selbstverwaltung wahr. Bei der Zulassung ambulant erbrachter Leistungen eines Krankenhauses, beispielsweise durch eine Ambulanz, müssen sie zustimmen. Auf Bundesebene bilden sie die Kassenärztliche und die Kassenzahnärztliche Bundesvereinigung.

Das zentrale Gremium der Selbstverwaltung ist nach $\$ 91$ SGB V der G-BA, in dem der GKV-Spitzenverband, die 
Deutsche Krankenhausgesellschaft sowie die Kassenärztliche und die Kassenzahnärztliche Bundesvereinigung vertreten sind. Der G-BA trifft im Auftrag des Gesetzgebers in allen Bereichen der Medizin versorgungsrelevante Entscheidungen durch den Erlass von verbindlichen Richtlinien und Beschlüssen. Die Aufgaben und Befugnisse des G-BA betreffen die gesamte medizinische und zahnmedizinische Versorgung zulasten der gesetzlichen Krankenversicherung. Der G-BA bewertet unter anderem neue medizinische Methoden hinsichtlich ihres diagnostischen und therapeutischen Nutzens sowie ihrer medizinischen Notwendigkeit und Wirtschaftlichkeit. Wichtige Aufgaben bestehen zudem im Erlass von Richtlinien zur Qualitätssicherung sowohl für die ambulante als auch für die stationäre Versorgung, in der Festlegung von Kriterien für die indikationsbezogene Notwendigkeit und Qualität der durchgeführten diagnostischen und therapeutischen Leistungen sowie in der Festlegung von Mindestanforderungen für die Struktur-, Prozess- und Ergebnisqualität. Die Qualitätsrichtlinien des G-BA gelten für Versicherte der gesetzlichen Krankenversicherung wie für Selbstzahler bzw. privat versicherte Patienten einheitlich.

Der G-BA hat ein unabhängiges und rechtsfähiges, jedoch selbst nicht entscheidungsermächtigtes wissenschaftliches Institut für Qualität und Wirtschaftlichkeit im Gesundheitswesen (IQWiG) gegründet, das er mit Recherchen, Bewertungen und der Abgabe von Empfehlungen beauftragen kann. Träger des Instituts ist eine vom G-BA eigens zu diesem Zweck errichtete Stiftung privaten Rechts.

Mit der Erstellung einrichtungsübergreifender und sektorenübergreifender Richtlinien zur Qualitätssicherung hat der G-BA im Jahr 2009 das fachlich unabhängige Beratungs- und Forschungsunternehmen Institut für angewandte Qualitätsförderung und Forschung im Gesundheitswesen (AQUAInstitut) betraut. Da sich die Regelung, wonach der G-BA in regelmäßigen Abständen ein aufwendiges europaweites Ausschreibungsverfahren für die Vergabe dieser Aufträge 
durchführen muss, als unzweckmäßig erwiesen hat, hat der Gesetzgeber im Jahr 2014 den Übergang zu einer dauerhaften Lösung beschlossen und den G-BA beauftragt, ein fachlich unabhängiges wissenschaftliches Institut für Qualitätssicherung und Transparenz im Gesundheitswesen (IQTIG) zu gründen. Dieses Institut wurde nach dem Vorbild des IQWiG im Januar 2015 geschaffen.

Das Krankenhausfinanzierungsgesetz ermächtigt den GKV-Spitzenverband, den Verband der Privaten Krankenversicherung sowie die Deutsche Krankenhausgesellschaft, die umfangreichen Arbeiten und Berechnungen, die mit dem gesetzlichen Auftrag zur Weiterentwicklung und Anpassung des DRG-Systems gemäß $\$ 17 \mathrm{~b}$ KHG verbunden sind, von einem eigenen DRG-Institut durchführen zu lassen. Mit der Wahrnehmung dieser Aufgaben, zu denen auch die in dem Gesetz vorgeschriebene Begleitforschung gehört, ist das InEK beauftragt worden.

\subsection{Gesetzgeberische Aktivitäten}

Das Krankenhausrecht ist geprägt von komplexen, teils unübersichtlichen Regelungsstrukturen einerseits und permanenten Interventionen und Nachjustierungen seitens des Gesetzgebers sowie der zahlreichen untergesetzlichen Normgeber andererseits. Bundesstaatliche Kompetenzverschiebungen, Strukturdebatten und Finanzierungsfragen bestimmen seit Jahrzehnten das Bild. Für die Akteure des stationären Versorgungssystems hat dies mangelnde Planungssicherheit und unsichere Orientierungsmaßstäbe zur Folge. Bis weit in die 1960er-Jahre war der Krankenhaussektor in Deutschland durch eine strukturelle Unterversorgung gekennzeichnet. Das Krankenhaus galt als „Aschenputtel des Wirtschaftswunders“. .55

55 So treffend Depenheuer 1986, 30 f.; zu einem knappen Überblick siehe etwa Quaas, in: Quaas/Zuck 2014, § 25 Rn. 12 ff. 
In dieser Situation entschloss sich die damalige Bundesregierung auf der Grundlage der sogenannten Krankenhaus-Enquete $^{56}$, die Verantwortung für die wirtschaftliche Sicherung der Krankenhäuser zu übernehmen und eine grundlegende Neuordnung der Krankenhausfinanzierung in Angriff zu nehmen. Bis dahin hatte dem Bund nach der grundgesetzlichen Kompetenzordnung lediglich die konkurrierende Gesetzgebungsbefugnis für das Sozialversicherungsrecht gemäß Art. 74 Nr. 12 GG (a. F.) zugestanden. Zwei Grundgesetzänderungen haben dem Bund gemäß Art. 74 Nr. 19a GG (a. F.) die konkurrierende Gesetzgebungszuständigkeit für die „wirtschaftliche Sicherung der Krankenhäuser und Regulierung der Krankenhauspflegesätze“ zugewiesen. Zugleich wurde dem Bund über Art. 104a Abs. 4 GG die Möglichkeit zur Beteiligung an der Finanzierung von Investitionen im Krankenhaus durch Finanzhilfen an die Länder eröffnet. Auf der Grundlage des neuen Art. 74 Nr. 19a GG verabschiedete der Deutsche Bundestag dann das Krankenhausfinanzierungsgesetz vom 29. Juni 1972. Die Krankenhausfinanzierung wurde damit nach dem Prinzip der dualen Finanzierung auf zwei Säulen gestellt. ${ }^{57}$ Die Investitionsförderung war zunächst Gegenstand einer Mischfinanzierung im Sinne einer zwischen Bund und Ländern geteilten Finanzierung. Die Beschränkung der Gesetzgebungskompetenz des Bundes auf die wirtschaftliche Sicherung der Krankenhäuser stellte aber zugleich klar, dass die Krankenhausplanung weiterhin eine Angelegenheit der Länder blieb. Mit Inkrafttreten der ersten Bundespflegesatzverordnung von 1973 wurde ein voll pauschaliertes System sogenannter tagesgleicher Pflegesätze eingeführt.

Im Wesentlichen waren damit die normativen Grundlagen gelegt für die Dreiteilung des Krankenhausrechts: (1) das

56 Deutscher Bundestag 1969.

57 Ob und in wieweit das geltende Verfassungsrecht ein (weitestgehend) monistisches Finanzierungssystem ermöglicht, ist umstritten. Siehe dazu beispielsweise Höfling 2007, 293 f.; Kaltenborn, in: Huster/Kaltenborn 2010, $\S 2$ Rn. 4 . 
Recht der Krankenhausplanung auf der Grundlage der $\$ \$ 6,8$ KHG sowie der von den Ländern jeweils erlassenen Landeskrankenhausgesetze ${ }^{58}$; (2) das Recht der Krankenhausfinanzierung nach Maßgabe des Krankenhausfinanzierungsgesetzes und der darauf gestützten Rechtsverordnungen, vor allem der Bundespflegesatzverordnung - ebenfalls ergänzt um die Landeskrankenhausgesetze; (3) das Leistungserbringerrecht im Rahmen der gesetzlichen Krankenversicherung namentlich auf der Grundlage des SGB V. Schon Anfang der 1980er Jahre setzte dann die sogenannte Kostendämpfungspolitik ein, die ab den 1990er-Jahren um eine „Strukturgesetzgebung“ ergänzt wurde. Als wichtige Stationen seien genannt:59

\D Das Krankenhaus-Kostendämpfungsgesetz ${ }^{60}$ vom 22. Dezember 1981 führte zu einer stärkeren Beteiligung der Krankenkassen und Krankenhausträger an der Krankenhausplanung der Länder. Die Investitionsförderung wurde durch Einteilung der Krankenhäuser in vier Versorgungsstufen neu geordnet; zugleich wurde die Verpflichtung der Länder statuiert, die Krankenhauspläne an den Entwicklungsbedarf der Krankenhausleistungen anzupassen. ${ }^{61}$

» Nach dem Regierungswechsel im Herbst 1982 setzte eine grundlegende Diskussion über die Reform der Krankenhausfinanzierung ein, die schließlich in das KrankenhausNeuordnungsgesetz ${ }^{62}$ vom 20. Dezember 1984 mündete. Mit der Reform wurde unter anderem die bisher im Investitionsbereich geltende Mischfinanzierung abgeschafft. Den Ländern oblag nunmehr alleine die Investitionsförderung.

58 Siehe dazu den Überblick bei Stollmann, in: Huster/Kaltenborn 2010, $§ 4$ Rn. $1,7 \mathrm{ff}$.

59 Die folgenden Darstellungen im Abschnitt 2.4 beruhen im Wesentlichen auf Tuschen/Trefz 2010, $44 \mathrm{ff}$.

60 Gesetz zur Änderung des Gesetzes zur wirtschaftlichen Sicherung der Krankenhäuser und zur Regelung der Krankenhauspflegesätze (BGBI. I, 1568), aufgelöst durch Art. 74 des Gesetzes vom 14. August 2006 (BGBI. I, 1869).

61 Siehe dazu und zum vorstehenden Thomae 2006, 8-13.

62 Gesetz zur Neuordnung der Krankenhausfinanzierung (BGBI. I, 1716). 
》) Mit dem Gesundheitsstrukturgesetz ${ }^{63}$ vom 21. Dezember 1992 wurde das in $\$ 4$ KHG normierte Selbstkostendeckungsprinzip aufgehoben. Nach der Neufassung der Norm wurde der Anspruch auf Deckung der vorauskalkulierten Selbstkosten ersetzt durch den Anspruch auf medizinisch leistungsgerechte Pflegesätze, die es einem Krankenhaus bei wirtschaftlicher Betriebsführung ermöglichen müssen, den Versorgungsauftrag zu erfüllen ( $\$ 17$ Abs. $1 \mathrm{KHG}$ ).

» Mit dem Gesundheitsstrukturgesetz in Verbindung mit der Bundespflegesatzverordnung vom 26. September 1994 wurden erstmalig pauschale Vergütungselemente eingeführt. In Abhängigkeit von der durchgeführten Prozedur konnten zwischen den Krankenhäusern und den Kostenträgern Fallpauschalen und Sonderentgelte vereinbart werden. Je nach Leistungsspektrum des Hauses betrafen die Pauschalen einen unterschiedlich hohen Anteil der behandelten Fälle. Durch die pauschale Vergütung war es für Krankenhäuser möglich, Verluste oder Gewinne zu erzielen.

»Mit Art. 3 des Beitragsentlastungsgesetzes ${ }^{64}$ vom 1 . November 1996 kam es zu einer Änderung von $\$ 17$ Abs. 3 KHG. Die Vertragsparteien wurden verpflichtet, zum Abbau von Fehlbelegungen die Krankenhausbudgets für die Jahre 1997 bis 1999 um mindestens ein Prozent pauschal zu senken, und zwar unabhängig davon, ob in einem bestimmten Krankenhaus tatsächlich Fehlbelegungen vorlagen oder nachgewiesen werden konnten.

》) Auch das Zweite GKV-Neuordnungsgesetz ${ }^{65}$ vom 23. Juni 1997, das rückwirkend zum 1. Januar 1997 in Kraft trat, brachte Änderungen im Krankenhausbereich. So kam es zu

63 Gesetz zur Sicherung und Strukturverbesserung der gesetzlichen Krankenversicherung (BGBI. I, 2266), zuletzt geändert durch Art. 205 der Verordnung vom 25. November 2003 (BGBI. I, 2304).

64 Gesetz zur Entlastung der Beiträge in der gesetzlichen Krankenversicherung (BGBI. I, 1631).

65 Zweites Gesetz zur Neuordnung von Selbstverwaltung und Eigenverantwortung in der gesetzlichen Krankenversicherung (BGBI. I, 1520), aufgelöst durch Art. 217 des Gesetzes vom 14. August 2006 (BGBI. I, 1869). 
einer Rückkehr bei der Vergütung der voll- und teilstationären Krankenhausleistungen zu einer leistungsorientierten Vergütung, wie sie vor 1995 bestand.

» Nach den Bundestagswahlen vom 27. September 1998 und dem folgenden Regierungswechsel wurde zur Vorbereitung einer größeren Reform und zur Unterbindung weiterer Beitragssatzsteigerungen kurzfristig das GKV-Solidaritätsstärkungsgesetz ${ }^{66}$ vom 19. Dezember 1998 als sogenanntes Vorschaltgesetz erlassen. Mit seinem Art. 7 wurde eine gesetzliche Begrenzung der Erlöse für stationäre Krankenhausleistungen im Jahr 1999 vorgeschrieben.

\D Das GKV-Gesundheitsreformgesetz $2000^{67}$ vom 22. Dezember 1999 bewirkte ebenfalls Änderungen des Krankenhausrechts. Als zusätzliche Form der Regelversorgung wurde unter anderem die integrierte Versorgung mit dem Ziel eingeführt, die sektoralen Grenzen zwischen stationärer und ambulanter Versorgung aufzulösen. Erstmals wurde die Möglichkeit geschaffen, Verträge zwischen den stationären und ambulanten Leistungserbringern und den Krankenkassen zur Versorgung bestimmter Patientengruppen unter pauschal kalkulierten Entgeltbedingungen zu vereinbaren. Darüber hinaus wurden die Krankenkassen zur internen Qualitätssicherung verpflichtet.

» Eine wesentliche Neuorientierung brachte die Einführung eines pauschalierten Entgeltsystems durch das sogenannte Fallpauschalengesetz ${ }^{68}$ vom 23. April 2002.69 Es folgte das

66 Gesetz zur Stärkung der Solidarität in der gesetzlichen Krankenversicherung (BGBI. I, 3853), zuletzt geändert durch Art. 20 Abs. 7 des Gesetzes vom 16. Juli 2015 (BGBI. I, 1211).

67 Gesetz zur Reform der gesetzlichen Krankenversicherung ab dem Jahr 2000 (BGBI. I, 2626), zuletzt geändert durch Art. 3 des Gesetzes vom 15. Februar 2002 (BGBI. I, 684).

68 Gesetz zur Einführung des diagnose-orientierten Fallpauschalensystems für Krankenhäuser (BGBI. I, 1412), zuletzt geändert durch Art. 3 des Gesetzes vom 17. Juli 2003 (BGBI. I, 1461).

69 Für die Krankenhäuser, die nicht in das DRG-System einbezogen sind, wie die psychiatrischen und psychosomatischen Krankenhäuser, gilt die Bundespflegesatzverordnung, mit der tagesgleiche Pflegesätze festgelegt werden. 
Fallpauschalenänderungsgeset $z^{70}$ vom 17. Juli 2003, das dem Bundesministerium für Gesundheit gegenüber den Vertragsparteien auf Bundesebene größere Regulierungsbefugnisse einräumte.

\) Auf der Grundlage des GKV-Modernisierungsgesetzes ${ }^{71}$ vom 14. November 2003 wurden den Krankenhäusern zusätzliche finanzielle Mittel unter anderem für die Verbesserung der Arbeitszeitbedingungen bereitgestellt. Des Weiteren wurde die integrierte Versorgung im SGB V neu geregelt. Die Zustimmungspflicht der Kassenärztlichen Vereinigung zu einem Vertrag zur integrierten Versorgung wurde abgeschafft und für die Finanzierung der Projekte zur integrierten Versorgung ein Prozent der Gesamtvergütung ambulanter und stationärer Leistungen zur Verfügung gestellt. $^{72}$

》Das Zweite Fallpauschalenänderungsgesetz ${ }^{73}$ vom 15. Dezember 2004 wollte einigen Mängeln wie der nicht sachgemäßen Abbildung der Maximalversorger begegnen und die Vereinbarung von Zusatzentgelten erleichtern. Zudem

70 Gesetz zur Änderung der Vorschriften zum diagnose-orientierten Fallpauschalensystem für Krankenhäuser (BGBI. I, 1461).

71 Gesetz zur Modernisierung der gesetzlichen Krankenversicherung (BGBI. I, 2190), zuletzt geändert durch Art. 1 des Gesetzes vom 15. Dezember 2004 (BGBI. I, 3445).

72 Derzeit gibt es etwa 5.500 Projekte zur integrierten Versorgung in Deutschland, die sich auf die verschiedensten Patientengruppen und Behandlungsformen beziehen. Da seit 2011 durch eine weitere Gesetzesveränderung auch die Liste der möglichen Vertragspartner erweitert wurde (unter anderem um die Träger ambulanter Rehabilitationseinrichtungen, Pflegeeinrichtungen und pharmazeutische Unternehmen), unterscheiden sich die Projekte auch stark hinsichtlich ihrer Inhalte, Strukturen und Finanzierungsformen. So gibt es unter anderem indikationsbezogene Verträge (zum Beispiel im Bereich der Endoprothetik), behandlungsbezogene Verträge (zum Beispiel ambulantes Operieren) oder Case-ManagementVerträge (transsektorale Versorgung). Die Finanzierungsvereinbarungen reichen von Budgetvereinbarungen über bevölkerungsbezogen-flächendeckende bis hin zu fallbezogenen Pauschalen. Nur ein Teil der Krankenhäuser nimmt an der integrierten Versorgung teil (2010 waren es laut dem "Sondergutachten 2012“ des Sachverständigenrates zur Begutachtung der Entwicklung im Gesundheitswesen 37,1 Prozent der Krankenhäuser).

73 Zweites Gesetz zur Änderung der Vorschriften zum diagnose-orientierten Fallpauschalensystem für Krankenhäuser und zur Änderung anderer Vorschriften (BGBI. I, 3429). 
wurde dem InEK die Aufgabe der Weiterentwicklung des Vergütungssystems zugewiesen.

》 Mit dem GKV-Wettbewerbsstärkungsgeset ${ }^{74}$ vom 26. März 2007 kam es zu Änderungen des Krankenhausfinanzierungsgesetzes (Art. 18), des Krankenhausentgeltgesetzes (Art. 19) und der Bundespflegesatzverordnung (Art. 20).

» Nach der Einführung und Etablierung des DRG-Vergütungssystems und der schrittweisen Angleichung der Krankenhausbudgets an ein landeseinheitliches Vergütungsniveau wurden dann mit dem Krankenhausfinanzierungsreformgesetz vom 17. März 2009 die gesetzlichen Vorgaben für den Regelbetrieb des DRG-Vergütungssystems ab dem Jahr 2009 angepasst.

Seit 2009 kam es im Durchschnitt jedes Halbjahr zu Änderungen unter anderem des Krankenhausfinanzierungsgesetzes, des Krankenhausentgeltgesetzes und des GKV-Versorgungsstrukturgesetzes. ${ }^{75}$

Mit dem Anfang August 2015 in weiten Teilen in Kraft getretenen GKV-Versorgungsstärkungsgesetz und dem Krankenhausstrukturgesetz sind erneut erhebliche strukturelle Veränderungen auch auf die Krankenhäuser zugekommen. So sollen die Bundesländer Qualitätsindikatoren in die

74 Gesetz zur Stärkung des Wettbewerbs in der gesetzlichen Krankenversicherung (BGBI. I, 378), zuletzt geändert durch Art. 4 des Gesetzes vom 28. Juli 2011 (BGBI. I, 1622).

75 Erwähnt seien hier nur die Änderung der $\$ \S 4,7,10$ und der Anlage 1 des Krankenhausentgeltgesetzes durch Art. 8 des GKV-Finanzierungsgesetzes vom 22. Dezember 2010 (BGBI. I, 2309), die Änderung der $\$ \S 10,17,17 \mathrm{a}, 17 \mathrm{~b}$ des Krankenhausfinanzierungsgesetzes durch Art. 6 bzw. der $\$ \S 2,21$ des Krankenhausentgeltgesetzes durch Art. 7 des GKV-Versorgungsstrukturgesetzes vom 22. Dezember 2011 (BGBI. I, 2983), die Änderung der $\$ \S 17,17 \mathrm{~b}-\mathrm{d}$, 18, 28 des Krankenhausfinanzierungsgesetzes durch Art. 1 bzw. der $\$ \S 2$, 4, 6, 8-10, 19, 21 des Krankenhausentgeltgesetzes durch Art. 3 des PsychEntgeltgesetzes vom 21. Juli 2012 (BGBI. I, 1613), die Änderung der $\S \S 4,6-10$ des Krankenhausentgeltgesetzes durch Art. 5a bzw. der $\$ \S 17 b$, 17c des Krankenhausfinanzierungsgesetzes durch Art. $5 \mathrm{c}$ des Gesetzes zur Beseitigung sozialer Überforderung bei Beitragsschulden in der Krankenversicherung vom 15. Juli 2013 (BGBI. I, 2423) und die Änderung der $\S \S 4,8,14$ des Krankenhausentgeltgesetzes durch Art. $2 \mathrm{~b}$ des Ersten Pflegestärkungsgesetzes vom 17. Dezember 2014 (BGBI. I, 2222). 
Krankenhausplanung aufnehmen können, die bei Nichterreichen zu Vergütungsabschlägen oder zum Ausschluss aus der Krankenhausplanung führen. Die Betriebskostenfinanzierung von Krankenhäusern soll durch Qualitätszuschläge, Zuschläge für die Notfallversorgung, Sicherstellungszuschläge, Zentrumszuschläge und krankenhausindividuelle Zuschläge verbessert werden. Bei planbaren Eingriffen, deren Anzahl durch Indikationserweiterung ohne größere Schwierigkeiten zu steigern ist, soll durch das Verfahren der ärztlichen Zweitmeinung und durch Qualitätssicherungsmaßnahmen eine Mengensteuerung erfolgen. Die Investitionsfinanzierung der Bundesländer soll dem Durchschnitt der in den Jahren 2012 bis 2014 aufgewendeten Mittel entsprechen. Ein mit 500 Millionen Euro ausgestatteter Strukturfonds hat den Abbau von Überkapazitäten, die stärkere Konzentration von Versorgungsangeboten und Umstrukturierungen in nicht akutstationäre Versorgungseinrichtungen zum Ziel. Ein PflegestellenFörderprogramm soll in den Jahren 2016 bis 2018 mit bis zu 660 Millionen Euro die Pflege im Krankenhaus stärken. Die Mehrausgaben sollen zu ca. 90 Prozent von der gesetzlichen Krankenversicherung getragen werden, die aber gleichzeitig durch die ebenfalls im Gesetz vorgesehene Mengensteuerung Einsparungen erwarten kann.

\subsection{Schlussfolgerungen}

Zusammenfassend lässt diese Übersicht erkennen, dass der Bereich der stationären Versorgung in Deutschland in operationaler, finanzieller, struktureller und rechtlicher Hinsicht eine höchst komplizierte Konstruktion darstellt, deren umfassendes Verständnis ein intensives Spezialwissen erfordert, das zudem wegen der systemimmanent hohen Dynamik erforderlicher Veränderungen einer andauernden Aktualisierung bedarf. Auf der Ebene der Leistungserbringer im ärztlichen Bereich, der Pflege und der im Krankenhaus tätigen Gesundheitsberufe 
ist ein stets aktuelles Wissen über die jeweils geltenden Details des Vergütungssystems gefordert, um abrechnungsrelevanten Defiziten in der Dokumentation von Diagnosen zu begegnen oder eine in ökonomischer Hinsicht problematische medizinische Leistungserbringung zu vermeiden. Diese Anforderung tritt zunehmend bestimmend neben eine qualitativ hochwertige und patientengerechte Versorgung. Auf der Systemebene legt bereits die offensichtliche Notwendigkeit fortlaufender gesetzlicher Nachbesserungen die Vermutung nahe, dass das gegenwärtige System der stationären Versorgung bisher keine Konsistenz und stabile selbsttragende Funktionalität entwickelt hat. Auch wenn durch gesetzgeberische Aktivitäten Verbesserungen erreicht wurden, wird deutlich, dass auf der Ebene der Krankenhäuser für die einzelnen Einrichtungen eine mittelfristige Planungssicherheit kaum existiert. Zudem treffen im System der stationären Versorgung unterschiedliche und teilweise gegensätzliche Anforderungen aufeinander und müssen vermittelt werden. Geht man vom Patientenwohl als ethischem Leitprinzip der Krankenhausbehandlung aus, stellt sich die Frage, wie sich dieses im Gesamtsystem der stationären Versorgung abbilden kann und welchen Anforderungen das System entsprechen muss, um diesem Leitprinzip gerecht zu werden. 


\section{PATIENTENWOHL ALS ETHISCHER MASSSTAB}

Die Aufgabe von Krankenhäusern ist es laut $₫ 107$ Abs. 1 SGB V, „Krankheiten der Patienten zu erkennen, zu heilen, ihre Verschlimmerung zu verhüten, Krankheitsbeschwerden zu lindern oder Geburtshilfe zu leisten“. Die Krankenhausversorgung soll dafür gemäß $₫ 1$ Abs. 1 KHG „bedarfsgerecht“ und „leistungsfähig“ sein. Diese Ziele wurden 2015 mit dem Krankenhausstrukturgesetz um die Ziele „qualitativ hochwertig“ und "patientengerecht" ergänzt. Auch wenn damit Fragen der genauen Definition aufgeworfen sind, machen diese Ergänzungen doch deutlich, dass die Behandlungsqualität und die Beachtung des individuellen Patientenbedarfs in Zukunft stärkeres Gewicht erhalten sollen.

Der Begriff der „patientengerechten Versorgung“ hebt dabei unter Berücksichtigung der allgemeinen Vorgaben des Gesundheitssystems wie dem Wirtschaftlichkeitsgebot ( $\$ 12$ SGB V) und der Beitragssatzstabilität ( $\$ 71$ SGB V) auf die Beachtung der Individualität des einzelnen Patienten und die Gewährleistung einer auf seinen Bedarf abgestimmten angemessenen Behandlung ab, die Zielbestimmung "qualitativ hochwertig" auf den dafür erforderlichen medizinischen Standard. Beide Elemente können als wesentliche Aspekte einer Patientenwohlorientierung angesehen werden.

Auch wenn die Orientierung am Patientenwohl als normativem Leitprinzip der Gesundheitsversorgung intuitiv plausibel erscheint, ist der Begriff des Patientenwohls nicht eindeutig definiert. Das Patientenwohl umfasst objektivierbare (medizinische Parameter, Zugänglichkeit der Leistung), subjektive (Behandlungszufriedenheit, Berücksichtigung subjektiver Präferenzen) und intersubjektive Dimensionen (wechselseitige Anerkennung, Respekt, Achtsamkeit). Sie stehen oft in einem Spannungsverhältnis. Mit den im Folgenden dargestellten drei ethischen Kriterien wird die Patientenwohlorientierung in 
normativer Hinsicht operationalisiert. Es handelt sich dabei um die selbstbestimmungsermöglichende Sorge, die Gewährleistung guter Behandlungsqualität und die Beachtung von Zugangs- und Verteilungsgerechtigkeit.

Unter diesen Kriterien der Patientenwohlorientierung genießt die selbstbestimmungsermöglichende Sorge einen Vorrang und wird als erste dargestellt, weil sie auf dem ethischen und verfassungsrechtlich relevanten Prinzip der Achtung der Selbstbestimmung einer Person ruht: Keine medizinische Behandlung darf unter Verletzung der Selbstbestimmung erfolgen. Das Zentrum von medizinischen Handlungen im stationären Bereich bildet zudem die Behandlungsqualität: Die Ermöglichung von Selbstbestimmung eines Patienten, die nicht auf eine ihm angemessene und qualitativ hochwertige Behandlung gerichtet wäre, würde ihr Ziel verfehlen. Weil Ressourcen nie unbegrenzt vorliegen, müssen sie gerecht und effizient verteilt werden. Ineffiziente und uneffektive Ressourcenallokation erzeugt Ungerechtigkeit und ist daher nicht nur aus ökonomischen, sondern auch aus ethischen Gründen zu vermeiden. Deshalb gehören Allokationsüberlegungen intrinsisch in solche ethischen Überlegungen; sie sind für die Erreichung des Patientenwohls ein notwendiges, wenn auch nicht hinreichendes Kriterium.

\subsection{Selbstbestimmungsermöglichende Sorge}

\subsubsection{Selbstbestimmung}

Der Begriff der Autonomie bezeichnet die grundsätzliche Fähigkeit des Menschen, aus eigenen Stücken vernünftige Erwägungen anzustellen, mit anderen Personen Gründe für Handlungen auszutauschen und Entscheidungen verantwortlich zu treffen. Diese Fähigkeit zeichnet den Menschen als moralfähiges Lebewesen aus. Hieraus wird ethisch und rechtlich 
das Recht auf Selbstbestimmung und auf die Entfaltung der Persönlichkeit ebenso abgeleitet wie die Verantwortung des Einzelnen für sein Handeln, für die ihn dabei leitenden Überzeugungen wie für dessen zurechenbare Folgen.

Selbstbestimmung bezeichnet vor dem Hintergrund, dass dem Menschen grundsätzlich Autonomie zukommt, die Möglichkeit der Realisierung von je eigenen Handlungsentwürfen und Handlungsentscheidungen. Die Realisierung dieser Möglichkeit hängt von konkreten Bedingungen ab. Sie beziehen sich auf Ort und Zeit des individuellen Lebens ebenso wie auf dessen Entwicklungsstadium. Auch die körperliche und seelische Gesundheit sind dafür von Bedeutung. Selbstbestimmung ist zugleich ein rechtlicher Anspruch, der in verschiedenen Rechtssystemen unterschiedlich ausgestaltet ist. ${ }^{76}$

Das Verständnis von Selbstbestimmung des Patienten umfasst verschiedene Aspekte. Um von einer selbstbestimmten Entscheidung sprechen zu können, muss eine Person über Handlungsmöglichkeiten („anders können“) verfügen, unter denen sie aufgrund von Überlegungen („Gründe haben“) wählen kann. Ferner erfordert Selbstbestimmung das Bewusstsein der eigenen Urheberschaft („Ich bin es“), das die Zurechenbarkeit einer gewählten Handlungsweise begründet.77

Die Ausübung von Selbstbestimmung setzt voraus, dass die Person die wesentlichen Aspekte, die ihre Entscheidungen leiten, ihrer Art und ihrer Tragweite nach versteht. Dies stellt hohe Anforderungen an die Kommunikation zwischen Arzt und Patient, wenn es etwa um die Einwilligung in medizinische Maßnahmen geht, aber auch an diejenige zwischen $\mathrm{Pa}$ tient und Pflegenden und anderen im Krankenhaus Tätigen, wenn es sich um Pflegehandlungen und die Gestaltung des Klinikalltags handelt. Selbstbestimmung erfordert des Weiteren, dass der Patient vor dem Hintergrund seiner Lebenssituation und seiner Einstellungen Behandlungsempfehlungen

76 Vgl. Deutscher Ethikrat 2013, 120.

77 Wunder 2008. 
oder Fragen zur Gestaltung pflegerischer Abläufe bewerten und seine Entscheidung danach ausrichten kann. Da es sich hierbei häufig um angst- oder schambesetzte Bewertungen und Entscheidungen handelt, setzt eine gute Kommunikation ein großes Maß an Empathie, Aufmerksamkeit, aber auch Wissen und Erfahrung der im Krankenhaus Tätigen voraus.

Von einem „Selbst“ eines Menschen kann sinnvoll nur in Relation zu anderen Menschen die Rede sein. Man kann sich selbst stets nur in Wechselwirkung zum jeweils anderen verstehen. Die die Achtung des anderen einschließende Selbstachtung fordert seine Anerkennung sowie Respekt und die Achtsamkeit im Umgang mit ihm ein. Hieraus ergibt sich auch die Grenze der Selbstbestimmung, nämlich dann, wenn ihre Durchsetzung die Rechte anderer verletzen würde. Im Krankenhaus, in dem es zahllose Formen von Krankheit und Not gibt und in dem überdies in zunehmender Anzahl Patienten anzutreffen sind, die kognitiv eingeschränkt sind und nur noch teilweise ohne Probleme über sich selbst bestimmen können, schließt der Respekt vor der Selbstbestimmung auch die Erwartung ein, Patienten in diesen Situationen zu stützen, zu fördern oder ihre Selbstbestimmungskompetenzen wiederherzustellen. Patienten, die nicht einwilligungsfähig und auf Bevollmächtigte oder Betreuer angewiesen sind, können mit entsprechender Unterstützung an den Entscheidungen beteiligt werden. Auch wenn ersetzende Entscheidungen von Bevollmächtigten oder Betreuern erforderlich sein sollten, ist die individuelle Einschätzung des Patienten zur Bestimmung seines jeweiligen Wohls von größter Bedeutung. Selbstbestimmung zu respektieren heißt darüber hinaus, auch Patienten, die ansonsten nur noch eingeschränkt oder nicht mehr für sich sprechen können, zu ermutigen, in kleinen Dingen wie der Alltagsgestaltung noch selbst Entscheidungen zu treffen.

Deshalb reicht es aus ethischer Perspektive nicht aus, nur auf dem rechtlich gebotenen Respekt vor der Selbstbestimmung eines jeden zu bestehen und ihn an der Ausübung seiner Selbstbestimmung nicht zu hindern. Es ist ethisch vielmehr 
geboten, sich um die Selbstbestimmung eines jeden Patienten zu bemühen. Dies wird besonders deutlich bei vulnerablen $\mathrm{Pa}-$ tientengruppen (siehe 4.5).

\subsubsection{Fürsorge und Sorge}

Die Art der Zuwendung zum Patienten im Kontext von Medizin und Pflege wird oft mit dem deutschen Wort der Fürsorge beschrieben. Dabei wird dieser Begriff im positiven Sinne als zugewandte, unterstützende Sorge verstanden, die jemanden in seiner Individualität und seinem Wertesystem wahrnimmt. Manche verbinden mit dem Begriff der Fürsorge allerdings, dass der Arzt oder Pflegende die Verantwortung für den $\mathrm{Pa}-$ tienten übernimmt, ohne dessen eigene Impulse, Anteile oder auch sein eventuelles Anders-Wollen wahrzunehmen. Diese heute gemeinhin als paternalistisch bezeichnete Haltung von Ärzten oder Angehörigen anderer Heilberufe basiert aufseiten der Handelnden meist nicht auf negativen Absichten, sondern eher auf der Vorstellung, das Beste für den Patienten zu tun. Auf dessen Seite wird solches Verhalten aber überwiegend als bevormundend, die eigene Person und die eigenen Vorstellungen ignorierend oder sogar abwertend erlebt.

Vor dem Hintergrund der im dargestellten Sinn negativen Konnotation des Begriffs Fürsorge wird im Folgenden der weithin als weniger belastet empfundene Begriff der Sorge verwendet, der die patientenzentrierte Haltung verdeutlichen soll. Der Begriff Sorge in diesem Sinne entspricht auch dem in 
der internationalen Literatur verwendeten Begriff Care ${ }^{78}$, der unter anderem folgende Dimensionen umfasst: mitmenschliche Zuwendung, Anteilnahme, Ermutigung (zur jeweils noch möglichen Selbstbestimmung). Damit wird dem Patienten Sicherheit und Vertrauen zu sich selbst gegeben, Anleitung (zu jeweils noch möglichen Aktivitäten des täglichen Lebens) und Unterstützung, aber auch Versorgung und die Übernahme von Verantwortung für den anderen, soweit dies erforderlich ist.

Die aus der Debatte um den Paternalismus bekannte Haltung der bevormundenden Verantwortungsübernahme ist grundsätzlich als Gefahr allerdings auch im Care-Konzept angelegt. Umso mehr kommt es darauf an zu überprüfen, wie sich die jeweiligen Beziehungen gestalten, und darauf zu achten, dass der andere als selbstkompetentes Subjekt anerkannt und in seiner Situation als eines geschwächten, auf Hilfe angewiesenen Mitmenschen ermutigend unterstützt wird. Beide Aspekte sind gleichermaßen wichtig und konstituieren das Konzept einer selbstbestimmungsermöglichenden Sorge.

\subsubsection{Arzt-Patient-Beziehung79}

In der Medizinethik wird der Patient heute in seiner doppelten Rolle als hilfebedürftiger Mensch und als „gestaltende Kraft“ ${ }^{\text {“8 }}$ angesehen, auf die sich die Ärzte, Therapeuten und Pflegenden

78 Hinter dem Begriff Care steht eine breite Debatte, deren Ausgangspunkt die moralpsychologischen Arbeiten Carol Gilligans bilden. Die meisten Care-Ethiken stellen Gerechtigkeit und Care einander gegenüber, was in der Debatte selbst kritisch diskutiert wird. Eine weitere Diskussion bezieht sich auf die Reproduktion klassischer Geschlechterstereotype in der Care-Ethik, die Konzentration auf den Nahraum der Beziehung, die enge Verbindung von Gefühl und moralischem Handeln u. Ä. Allerdings gibt es auch Ansätze, die eine „öffentliche Care-Ethik“ konzipieren, in der die Bedingungen reflektiert werden, die eine gerechte Gesellschaft braucht, um Sorge oder Fürsorge im Sinne von Care leisten und deren Verwirklichung im Beziehungsraum ermöglichen zu können. Für eine gute Zusammenfassung der feministisch-ethischen Kritik einer Care-Ethik siehe Jaggar 1995.

79 Das hier Gesagte gilt selbstverständlich auch für andere Behandler-PatientVerhältnisse.

80 Reiter-Theil 2000, 844 . 
bei der Gestaltung ihrer Beziehung zu dem Patienten einzustellen haben. Einerseits treten Patienten heute dem Arzt oder dem Pflegenden selbstbewusst und informiert, mit eigener Verantwortung, mit Rechten und Pflichten gegenüber. ${ }^{81}$ Andererseits muss stets die besondere Situation der Patienten im Krankenhaus gesehen werden, die, anders als in der ambulanten Versorgung, nicht nur oft durch ihre Erkrankung, sondern meist auch schon durch den besonderen Umstand, in der ungewohnten Situation eines Krankenhauspatienten zu sein, auf besondere Hilfe angewiesen sind. Im Falle einschränkender Affekte wie Angst und Scham oder eingeschränkter kognitiver Fähigkeiten braucht er Unterstützung, um für sich selbst zu entscheiden oder, soweit es geht, an Entscheidungen teilzuhaben.

Das Patientenrechtegesetz hebt einen weiteren Aspekt hervor. Danach sollen Behandelnder und Patient zusammenwirken. Schon vor Inkrafttreten des Gesetzes wurde der Gedanke einer Mitwirkungspflicht des Patienten diskutiert. Sie wird häufig als Pflicht des Patienten zur Compliance im Sinne von Regelbefolgung verstanden. In der Definition der Weltgesundheitsorganisation von 2001 wurde darunter „das Ausmaß, in welchem ein Patient medizinische Instruktionen befolgt ${ }^{\text {"82 }}$, verstanden. In der heutigen Sicht auf den Patienten als selbstbestimmt handelndes Subjekt wäre es jedoch angemessen, das Adhärenz-Konzept zu verfolgen. Adhärenz bezeichnet das

81 Dieser Sichtweise trägt auch das Gesetz zur Verbesserung der Rechte von Patientinnen und Patienten vom 20. Februar 2013 (BGBI. I, 277) Rechnung. Das Arzt-Patient-Verhältnis wird im Patientenrechtegesetz als eigener Vertrag festgeschrieben. Die Rechte und Pflichten werden auch auf die nicht ärztlichen Heil- und anderen Gesundheitsberufe ausgedehnt. Mit dem Gesetz werden die zuvor in der Rechtsprechung entwickelten Behandlungsgrundsätze kodifiziert. Dazu gehören vor allem: das Erfordernis der Behandlung nach den zum Zeitpunkt der Behandlung bestehenden, allgemein anerkannten fachlichen Standards; der Anspruch auf eine angemessene Aufklärung und Beratung; das Erfordernis der Einwilligung in die Behandlung; das Erfordernis der Dokumentation und das Recht auf Einsicht in die Patientenakte.

82 Ins Deutsche übersetzt. Im Original: „the extent to which the patient follows medical instructions" (Sabaté 2001, 7). 
Ausmaß, in dem das Verhalten des Patienten mit einem gemeinsam abgesprochenen Therapiekonzept übereinstimmt. Es berücksichtigt damit sehr viel stärker die jeweils individuellen Faktoren des Verstehens und der Mitwirkungsmöglichkeiten. Im Gegensatz zum Compliance-Konzept wird somit von der Einbeziehung des Patienten und der Förderung des Selbstpflegemanagements ${ }^{83}$ in der Gestaltung der Therapie ausgegangen.

Das Adhärenz-Konzept greift auch das Verständnis von Gesundheitskompetenz des Patienten besser auf als das Compliance-Konzept. Unter Gesundheitskompetenz wird dabei neben dem Wissen und der Motivation die Kompetenz verstanden, Gesundheitsinformationen zu erlangen, zu verstehen, zu beurteilen und umzusetzen. ${ }^{84}$

In diesem Zusammenhang ist die Gestaltung der jeweiligen Arzt-Patient-Beziehung von großer Bedeutung, da sie in mehr oder weniger ausgeprägter Weise an der Gesundheitskompetenz des Patienten ansetzt. Die nachfolgenden Aussagen treffen in ihrer grundsätzlichen Art ebenso auf die PflegendePatient-Beziehung oder Therapeut-Patient-Beziehung zu. Neben dem heute größtenteils überwundenen paternalistischen Modell werden folgende Gestaltungsformen diskutiert:

\) das informative Modell (informative model): dem Patienten werden alle Informationen zur Verfügung gestellt, ihm wird aber in der Annahme, dass er seine Erwartungen und Werte selbst am besten einschätzen kann, die Entscheidung überlassen,

» das interpretative Modell (interpretative model): der Arzt stellt nicht nur alle Informationen zur Verfügung, sondern

83 Unter Selbstpflegemanagement ist hier die Aktivität des Patienten zu verstehen, mit der er Zeichen und Symptome einer Verschlechterung einer Erkrankung auf ihre Bedeutung überprüft und Maßnahmen zur Vermeidung einer weiteren Verschlechterung beispielsweise durch Hinzuziehung professioneller Hilfe einleitet.

$84 \mathrm{Vgl}$. Sørensen et al. 2012. 
befragt und berät den Patienten zu seinen Werten und Erwartungen und spricht durchaus auch Empfehlungen aus,

» das abwägende Modell (deliberative model): auf der Grundlage von Information, Beratung und einer gemeinsamen Exploration der gesundheitsbezogenen Werte wird der Patient ermutigt, im Rahmen der Indikationsstellung eine gute Entscheidung zu treffen. ${ }^{85}$

Das Abwägungsmodell kommt dem heute überwiegend empfohlenen Modell der partizipativen Entscheidungsfindung (shared decision making) am nächsten. Die partizipative Entscheidungsfindung setzt aber noch deutlicher auf die Gleichberechtigung von Arzt und Patient und das Aushandeln einer Entscheidung auf der Grundlage bestmöglicher Information und Berücksichtigung der Werte und Erwartungen des $\mathrm{Pa}$ tienten. ${ }^{86}$ Es hat damit eine gelingende Kommunikation zur Voraussetzung, beinhaltet unter anderem Vereinbarungen zur Umsetzung der Entscheidung und liegt dem Konzept der Adhärenz zugrunde.

\subsubsection{Gelingende Kommunikation als Voraussetzung selbstbestimmungs- ermöglichender Sorge}

Beziehungen von Patienten zu ihren Ärzten, Pflegenden und Therapeuten sind auf eine gelingende Kommunikation angewiesen. Kommunikationskompetenz ist eine unverzichtbare Schlüsselkompetenz aller Heilberufe. Sie umfasst neben der Kommunikation mit dem Patienten auch die Kommunikation mit den Personen im Umfeld des Patienten, denen insbesondere nach der Krankenhausentlassung eine große Bedeutung bei der Absicherung von Therapieerfolgen zukommt.

85 Klemperer 2003, 14 ff.; Emanuel/Emanuel 1992.

86 Loh et al. 2007. 
Empirische Untersuchungen belegen jedoch zahlreiche Defizite in diesem Bereich und offenbaren erhebliche Unzufriedenheit aufseiten der Patienten. ${ }^{87}$ Studien zeigen, dass die Informationen über medizinische Maßnahmen $\mathrm{zu}$ wichtigen Faktoren für Patientenzufriedenheit gehören. ${ }^{88}$ Eine defizitäre Kommunikation ist nicht nur Anlass für Patientenunzufriedenheit, sondern führt bei 19 Prozent der Fälle zu fehlender Adhärenz. ${ }^{89}$ Dieses wichtige Thema und die daraus resultierenden Fragen waren Gegenstand kontroverser Diskussionen beim 118. Deutschen Ärztetag im Jahr 2015. Im Beschlussprotokoll des Ärztetages wurde deshalb der zentrale Stellenwert der Kommunikation im Gesundheitswesen ausdrücklich betont und die dringende Forderung nach zahlreichen notwendigen Verbesserungen in diesem Bereich erhoben. ${ }^{9 \circ}$

Eine gelungene Aufklärung liefert dem Patienten zunächst notwendige Informationen für seine Entscheidungen in einer von ihm oftmals als Grenzsituation empfundenen Lage. ${ }^{91}$ Die richtige Einschätzung der Informationsbedürfnisse des Patienten ist in diesem Zusammenhang von großer Bedeutung. Ärztliche und pflegerische Aufklärung sollte jedoch keineswegs reduziert werden auf ein berufliches Ritual des Informierens zu Behandlungsbeginn. Vielmehr gehört zu einer selbstbestimmungsermöglichenden Sorge auch die Vergewisserung, dass der Patient die Informationen verstanden hat, sowie das Gespräch über die Bewertung durch den Patienten vor dem Hintergrund seiner Werte und Präferenzen. Zudem ist die Kommunikation zwischen Behandelndem und Behandeltem als ein Prozess während der gesamten Behandlungsdauer zu verstehen. Dabei sind auch die Entwicklungen aufseiten des Patienten im Laufe der Behandlungsphasen zu berücksichtigen.

87 Lahmann/Dinkel 2014.

88 Schoenfelder/Klewe/Kugler 2011.

89 Haskard Zolnierek/DiMatteo 2009.

90 Bundesärztekammer 2015a, 99.

91 Geisler 2010. 
Für eine gelingende Kommunikation sind neben dem Inhalt der Gespräche auch die Art und die Rahmenbedingungen entscheidend. Tugenden und Kompetenzen wie Wohlwollen, Aufrichtigkeit, Einfühlsamkeit, Zuwendung und Orientierung auf größtmögliche Selbstständigkeit sollten den gesamten Kommunikationsprozess prägen..$^{92} \mathrm{Um}$ die Schweigepflicht $\mathrm{zu}$ wahren und die erforderliche Zuwendung in bestimmten Situationen - beispielsweise bei Mitteilung einer Krebsdiagnose - zu gewährleisten, sind geeignete räumliche, zeitliche und personelle Ressourcen bereitzuhalten. Durch das Training kommunikativer Kompetenzen und das Vermitteln nützlicher Konzepte für eine gute und zielführende Gesprächsgestaltung kann die zur Verfügung stehende, oft knappe Zeit für ein Gespräch sehr viel effektiver gestaltet werden. ${ }^{93}$

Eine besondere Herausforderung kann die Kommunikation mit Patienten darstellen, die besonderer Zuwendung bedürfen. Hierzu gehören Kinder und Jugendliche, Patienten in hohem Lebensalter, mit geriatrietypischen Erkrankungen, mit Behinderung, mit Demenz, sowie Patienten anderer ethnischer Herkunft, Nationalität oder Religionszugehörigkeit. Interkulturelle Behandlungssituationen sind dabei oft nicht nur durch sprachliche Barrieren, sondern auch durch kulturelle Unterschiede beeinträchtigt, die in einem ethisch angemessenen Entscheidungsprozess die Berücksichtigung differierender moralischer Wertvorstellungen erfordern.

Zusammenfassend lässt sich festhalten, dass die für das Patientenwohl notwendige selbstbestimmungsermöglichende Sorge die Respektierung und Achtung des Patienten als Person mit eigenen Vorstellungen, Wünschen, Interessen, einer eigenen Geschichte und mit eigenen Rechten zum Ausgangspunkt hat. Die Selbstbestimmung des Patienten und damit seine Entscheidungen nach ausführlicher Information zu respektieren, stellt die unumgängliche Voraussetzung der Behandlung dar. 
Eine selbstbestimmungsermöglichende Sorge umfasst auch Ermutigung und Unterstützung, wenn der Patient durch Affekte wie Angst oder Scham oder durch kognitive Einschränkungen zu einer selbstbestimmten Entscheidung nur eingeschränkt fähig ist. Voraussetzung hierfür ist stets ein Arzt-Patient- bzw. Pflegende-Patient- oder Therapeut-Patient-Verhältnis, das von Vertrauen und Empathie getragen wird und zugewandte Kommunikation, partnerschaftliche Entscheidungsprozesse und Therapieabsprachen im Sinne des Adhärenz-Konzepts umfasst.

\subsection{Behandlungsqualität}

Eine weitere Maßstabsgröße für das Wohl des Patienten ist die Behandlungsqualität.

Die Qualität lässt sich dabei unterteilen in objektive Kriterien nach medizinisch-wissenschaftlichen Gesichtspunkten, etwa Besserung oder Heilung der Krankheit, Linderung von Schmerzen und anderen Symptomen, Verbesserung oder Wiederherstellung der Funktionalität in allen Lebensbereichen, sowie in subjektive Kriterien wie die Lebensqualität, die Patientenzufriedenheit und die Übereinstimmung eigener Ziele mit dem Behandlungsergebnis. Die Definition geeigneter Kriterien und Methoden für eine Messung der Behandlungsqualität stellt eine große Herausforderung dar, die für das Krankenhaus eine zunehmende Bedeutung gewinnt, weil gemäß dem Krankenhausstrukturgesetz die Höhe der Vergütung für stationäre Behandlungen perspektivisch an der Behandlungsqualität orientiert wird. ${ }^{94}$

94 Der durch Art. 1 Nr. 2 des Krankenhausstrukturgesetzes neu ins Krankenhausfinanzierungsgesetz eingefügte $\S 6 \mathrm{Abs}$. 1a legt fest, dass der G-BA die bisher nur in $\$ 136 \mathrm{c}$ Abs. 1 SGB V explizit erwähnten Qualitätsdimensionen von Donabedian (1966; 1980) nun auch bei der Qualitätssicherung im Krankenhaus zugrunde legen soll. Durch Länderentscheidung können diese aber auch ausgeschlossen werden und andere Dimensionen eingeführt werden. 


\subsubsection{Qualitätsmodell und Operationalisierung}

Qualitätsmessung und Qualitätssicherung im Gesundheitswesen orientieren sich überwiegend am Modell von Struktur-, Prozess- und Ergebnisqualität nach Avedis Donabedian. ${ }^{95}$ In diesem Modell werden dem Begriff der Behandlungsqualität alle drei Qualitätsdimensionen zugeordnet und ein ganzheitlicher Qualitätsansatz vertreten. Die Beurteilung der Notwendigkeit und der Art einer Behandlung betreffen alle drei Qualitätsdimensionen und basieren in vielen Fällen auf empirisch gewonnenen, statistisch gesicherten und durch Erfahrungswissen fundierten medizinischen Regeln. Für zahlreiche Behandlungsprozeduren existieren evidenzbasierte medizinische Leitlinien, die zumeist von medizinischen Fachgesellschaften entwickelt werden und den Anspruch erheben, auf der Basis von statistisch gesicherten Daten und Erfahrungswissen allgemein akzeptiert zu sein, fortlaufend überprüft $\mathrm{zu}$ werden und damit den aktuellen wissenschaftlich begründeten medizinischen Standard zum Ausdruck zu bringen. ${ }^{96}$ Vorbehaltlich medizinisch begründeter Abweichungen im Einzelfall oder neuer wissenschaftlicher Erkenntnisse stellt die gewissenhafte Beachtung dieser medizinischen Standards bei der Behandlung im stationären Bereich eine Voraussetzung für eine qualitativ hochwertige Behandlung dar. Patienten dürfen erwarten, dass diese Standards im Krankenhaus eingehalten werden, und Krankenhäuser müssen die Einhaltung dieser Standards in der Einrichtung ermöglichen, prüfen und sicherstellen.

Für viele Behandlungsszenarien, insbesondere bei selteneren Erkrankungen, existieren allerdings keine Leitlinien. In diesen Fällen und auch in allen Behandlungsfällen mit einer größeren Vielfalt möglicher Behandlungsoptionen muss der Behandelnde auf der Grundlage von Behandlungsanalogien, fachinterner Übereinkunft und eigenem Erfahrungswissen

95 Donabedian 1966; Donabedian 1980.

96 Näher Taupitz 2009. 
eine Entscheidung treffen, deren Grundlagen er dem Patienten offenlegen muss. Insofern bedürfen diese Situationen der ärztlichen Expertise und Sorgfalt. Gegebenenfalls sind interdisziplinäre Absprachen und das Einholen von Spezialexpertisen oder Zweitmeinungen notwendig, um eine qualitativ hochwertige Behandlung zu ermöglichen.

Da die überwiegende Mehrzahl der Leitlinien nicht für besondere Patientengruppen, wie ältere und hochbetagte Patienten, multimorbide und chronisch kranke Patienten mit funktionellen Einschränkungen (zum Beispiel Hör- und Sehminderung), Patienten mit Mild Cognitive Impairment, verschiedenen Demenzformen und -stadien, Patienten mit unterschiedlichen Behinderungen und Patienten mit seltenen Erkrankungen, erarbeitet wurden, ist der Arzt auch hier oft auf Entscheidungen angewiesen, die auf der Grundlage der oben genannten verschiedenen Quellen getroffen werden müssen.97

\section{Strukturqualität}

Unter Strukturqualität versteht man die Qualität der zur Verfügung stehenden Mittel, wie räumliche Bedingungen, apparative Ausstattung des Krankenhauses, aber auch Ausbildungsstand und Personalausstattung. Für eine gute Behandlungsqualität ist es erforderlich, dass ein Krankenhaus und seine Mitarbeiter eine Patientenbehandlung nur dann übernehmen, wenn

》) die Raumsituation keine besonderen gesundheitsgefährdenden Risiken birgt (zum Beispiel Hygienemängel),

») die Medizintechnik dem Diagnostik- und Therapiebedarf entspricht,

» das Personal in ausreichender Anzahl und mit der erforderlichen Qualifikation bei der Behandlung eingesetzt werden kann.

97 Aylett 2010. 
Sofern die Strukturqualität in einer Einrichtung dem spezifischen Bedarf eines Patienten nicht entspricht, sind die Voraussetzungen für seine Behandlung in dieser Einrichtung nicht erfüllt.

\section{Prozessqualität}

Die Prozessqualität bezieht sich auf die Frage, wie die richtigen Behandlungsschritte gewählt werden (Indikationsstellung, Effektivität) und die zur Behandlung erforderlichen Teilschritte sinnvoll organisiert werden (Effizienz).

Zur Sicherstellung einer hohen Behandlungsqualität ist es wichtig, dass

\) die medizinische Indikation in angemessener Zeit gemäß dem aktuellen Stand der medizinischen Wissenschaft gestellt und nur auf dieser Grundlage eine Behandlung begonnen wird,

» die einzelnen Behandlungsschritte sinnvoll organisiert sind und unnötige Wartezeiten, Doppeluntersuchungen bzw. nicht erforderliche Untersuchungen vermieden werden,

» der Patient während seiner Behandlung in adäquater Weise informiert und in Entscheidungen einbezogen wird.

\section{Ergebnisqualität}

In Bezug auf die Ergebnisqualität finden sich für einige, aber nicht alle Parameter festgelegte Kennzahlen. Beispiele sind Mortalitäts-, Komplikations-, Dekubitusraten oder Stürze im Krankenhaus. Neben der medizinischen und pflegerischen Bewertung sind Ergebnisse aus Patientenbefragungen ebenfalls ein Element der Ergebnisqualität. Mängel in der Ergebnisqualität sind größtenteils auf Defizite bei den Struktur- und Prozessvoraussetzungen zurückzuführen. $\mathrm{Zu}$ einer guten Behandlungsqualität gehört, dass 
» zu Kennzahlen der Ergebnisqualität Transparenz und Vergleichbarkeit geschaffen wird,

» das Krankenhaus eine konstruktive Fehlerkultur zulässt und diese in ein Risikomanagement zur kontinuierlichen Verbesserung der Ergebnisqualität integriert.

\subsubsection{Qualitätsmessung}

Der Begriff der qualitativ hochwertigen Versorgung im Krankenhaus umfasst somit neben der Qualität der medizinischen und pflegerischen Behandlung eine Vielzahl baulicher, ausstattungsbezogener und prozedural kommunikativer Aspekte, denen in Zukunft auf der Grundlage des 2015 verabschiedeten Krankenhausstrukturgesetzes ein großes Gewicht zugemessen wird. Die große Herausforderung besteht bei der neu eingeführten Qualitätsorientierung in der Krankenhausplanung (siehe 2.4) für den G-BA darin, evidenzbasierte und gerechte Qualitätsindikatoren zu entwickeln, die - möglichst bundesweit einheitlich - umsetzbar, messbar, vergleichbar und justiziabel sind.

Die Qualitätsdimensionen lassen sich unterschiedlich gut messen. Während die Strukturqualität noch relativ leicht zu bewerten ist, indem geprüft wird, ob geforderte sachliche oder personelle Ressourcen zur Verfügung stehen, ist dies bei der Prozessqualität schon deutlich schwieriger. Zur Bewertung müssten auswertbare Daten zu einzelnen Teilschritten über die gesamte Behandlung (auch unter Berücksichtigung von Schnittstellen zu anderen Leistungserbringern außerhalb des Krankenhauses) mit Angabe von Zeitpunkten vorliegen. Dies ist nur für wenige Teilbereiche, wie zum Beispiel den Operationssaal, gegeben. Die größten Bewertungsprobleme bestehen hinsichtlich der Ergebnisqualität. Ursächlich hierfür sind gegebenenfalls Unterschiede zwischen der subjektiv empfundenen und objektiv nachvollziehbaren Qualität sowie die sich im individuellen Behandlungsverlauf verändernde Bewertung 
bzw. die Frage nach dem geeigneten bzw. angemessenen Zeitpunkt für eine Qualitätsmessung. Angemessene Indikatoren für die Erhebung und Interpretation der Ergebnisqualität zu finden, wirft zusätzliche Schwierigkeiten auf: Auf das Ergebnis wirken sich auch die individuelle gesundheitliche Situation des Patienten, seine Erwartungen, gegebenenfalls vorangegangene Behandlungen sowie die Adhärenz des Patienten während der Behandlung aus.

Zusammenfassend lässt sich feststellen, dass eine die objektiven wie subjektiven Dimensionen umfassende hochwertige Behandlung im Krankenhaus ein zentrales Kriterium für das Patientenwohl darstellt. Sie verbindet sich mit dem Kriterium der selbstbestimmten Einwilligung des Patienten und deren Ermöglichung durch eine auf die Selbstbestimmung gerichtete Sorge. Für eine qualitativ hochwertige und patientengerechte Behandlung kommt der verantwortungsvoll gestellten, auf den individuellen Patienten bezogenen medizinischen Indikation eine grundlegende Bedeutung zu. Auch die Bundesärztekammer bezieht in ihrer Stellungnahme „Medizinische Indikationsstellung und Ökonomisierung "98 die medizinische Indikation eindeutig auf das Patientenwohl, das sie allerdings durch zunehmend ökonomisch definierte Zielvorgaben, die als medizinfremde Beurteilungskriterien in die Indikationsstellung einfließen, bedroht sieht. Letztlich sind die für eine indikationsgerechte Behandlung erforderlichen Strukturen und Prozesse im Krankenhaus von entscheidender Bedeutung für eine hochwertige Qualität der Behandlungsergebnisse.

98 Bundesärztekammer 2015b. 


\subsection{Gleicher Zugang zu Gesundheits- leistungen und gerechte Verteilung von Ressourcen}

Gerechtigkeit meint formal zunächst die gerechtfertigte Berücksichtigung des jedem jeweils Angemessenen. Von alters her wird um die konzeptionelle Ausdifferenzierung dieser für menschliches Zusammenleben grundlegenden Forderung gerungen. Seit Aristoteles verlangt die Gerechtigkeit einige Minimalstandards, die man als formale oder universale Gerechtigkeit bezeichnen kann und der man Elemente des fairen Verfahrens, der gleichen Behandlung gleicher Fälle oder der Beachtung notwendiger Transparenz zuordnen kann. ${ }^{99}$ Der Gedanke der formalen Gerechtigkeit lässt sich in der Moderne über diese Elemente hinaus so ausdehnen, dass zu den Mindestbedingungen einer gerechtfertigten und gleichmäßigen Berücksichtigung des jedem jeweils Angemessenen die gleiche Achtung als Person (Statusgleichheit; status equality) gehört. ${ }^{100}$

Menschen begegnen einander unter Bedingungen erheblicher Ungleichheit. Ohne auch nur ansatzweise die breite Diskussion über Notwendigkeit und Grenzen der gesellschaftlichen Reaktion auf solche sozialen Ungleichheiten hier diskutieren zu können, lässt sich doch festhalten, dass sich ein breiter Konsens darüber etabliert hat, dass jeder Mensch die faire Chance haben sollte, seine eigene Persönlichkeit im Rahmen der gegebenen gesellschaftlichen Rahmenbedingungen entwickeln und am gesellschaftlichen Leben teilhaben zu können. Vor diesem Hintergrund legitimieren unterschiedliche

99 Zum Begriff der Gerechtigkeit bei Aristoteles siehe Fünftes Buch der Nikomachischen Ethik.

100 Der Gleichheitsgrundsatz im Sinne der equality beim Zugang zur Gesundheitsversorgung wird maßgeblich geprägt durch eine basale Statusgleichheit aller Menschen. Sie ist Ausdruck der objektiv-rechtlichen Dimension der Menschenwürdegarantie des Art. 1 Abs. 1 GG und wird untermauert durch den allgemeinen Gleichheitssatz des Art. 3 Abs. 1 GG (vgl. Kirchhof, in: Isensee/Kirchhof 1992, § 124 Rn. 199). 
Bedarfe unterschiedliche Zuteilung von Ressourcen, um Chancengerechtigkeit überhaupt erfahren zu können.

Ferner wird man unter den Bedingungen knapper Ressourcen kaum bestreiten können, dass eine ineffiziente und ineffektive Mittelverwendung ungerecht ist. Dass die Bestimmung, was Effizienz und Effektivität letztlich ausmacht, hochgradig umstritten ist, ändert nichts an der generellen Feststellung, dass an Effizienz und Effektivität ausgerichtete Wirtschaftlichkeitsüberlegungen Elemente von Gerechtigkeit sind.

Im Hinblick auf die Krankenhausversorgung ist dabei sowohl der Zugang zu Krankenhausleistungen zu betrachten als auch die Zuteilung von Leistungen im Krankenhaus. Für den Begriff der Gleichheit, der in der Debatte über Gerechtigkeit eine entscheidende Rolle spielt, wird vorgeschlagen, zwischen Gleichberechtigung im Sinne des Grundrechts (equality) und angemessener Berücksichtigung der Besonderheiten des Individuums im Sinne der fairen Behandlung (equity) zu unterscheiden. ${ }^{101}$

Das Kriterium der Gleichberechtigung bezieht sich in diesem Sinne auf eine statusindifferente Krankenhausversorgung. Es sperrt sich grundsätzlich gegen die Exklusion bestimmter Patientengruppen. Diese prinzipiellen Feststellungen bedeuten indes nicht, dass jedes einzelne Krankenhaus gleichermaßen die Zugangsmöglichkeit für alle ungeachtet der ökonomischen Konsequenzen gewährleisten muss. Vielmehr darf der die Gewährleistungsverantwortung tragende Staat durchaus Angebote bündeln oder bestimmte Versorgungsleistungen stärker zentralisieren, um so eine bedarfsgerechte Krankenhausversorgung sicherzustellen, die auch den speziellen Anforderungen besonderer Patientengruppen zugute kommt. Erforderlich sind Regeln, die ungerechtfertigte Benachteiligungen und Privilegierung verhindern.

Gerechtigkeit im Sinne der fairen Behandlung zeigt sich bei der Bereitstellung von jeweils erforderlichen

101 Satzinger/Werner 2005, $115 \mathrm{ff}$. 
Gesundheitsleistungen vor dem Hintergrund begrenzter Ressourcen. Hier geht es nicht um eine Tauschgerechtigkeit (wie im Wirtschaftsleben) oder um die ausgleichende Gerechtigkeit (wie im Schadensersatz- oder im Strafrecht). Zwar haben Versicherte durch Beiträge an die Krankenversicherung einen Anspruch auf eine angemessene Gesundheitsversorgung, aber die Art und der Umfang der zu erbringenden Leistung richten sich nicht nach einem Beitragsprinzip. Es geht auch nicht um eine Zuteilung identischer Anteile, wie es das Gleichheitsprinzip im Sinne von equality nahelegen würde. Für die zu erbringenden Leistungen ist der Bedarf bzw. Zustand des Patienten maßgeblich, dessen Erkrankung und Behandlung ganz individuelle Anforderungen stellen (Bedarfsprinzip). In diesem Zusammenhang kann eine ungleiche Verteilung von medizinischen Ressourcen auch dann als gerecht gelten, wenn sie zur Gleichheit von Lebenschancen beiträgt, die durch Krankheit und Behinderung ungleich verteilt sind. ${ }^{102}$

Allerdings stößt der Versuch, eine gerechte Ressourcenallokation im Gesundheitswesen im Allgemeinen und bei der Verwendung von Krankenhausleistungen im Besonderen zu bewirken, auch dann an Grenzen, wenn man sich von equality, equity und Kosten-Nutzen-Analysen leiten lässt. Irgendwann erreicht man unter Budgetbegrenzungsbedingungen den Punkt, an dem sich ein Konflikt zwischen fairen Chancen und bestem Outcome abzeichnet, ja unvermeidbar wird. ${ }^{103}$

Was alle diese Fragen zeigen, ist das Folgende: Die Verbindlichkeit selbst strikt generalisierender Kriterien wie Statusgleichheit, Zugang, Sensibilität für chancenmindernde Benachteiligungen, Effizienz und Effektivität schließt die Notwendigkeit von Erwägungen zu unterschiedlichen Vorstellungen vom "guten Leben“ und $\mathrm{zu}$ den zugehörigen

102 Vgl. Daniels 2013; Daniels 1996, 191 ff.; ausführlich: Daniels 2008, 29-56. Das hauptsächliche Argument: "Since meeting health needs promotes health (or normal functioning), and since health helps to protect opportunity, then meeting health needs protects opportunity" (Daniels 2008, 30).

103 Vgl. dazu und zum folgenden Beispiel Brock/Wikler 2006. 
Menschenbildern nicht aus. Auch solche Einstellungen wirken sich darauf aus, was unter dem Gerechtem und unter dem Verallgemeinerbaren verstanden wird. Wo solche Prägungen transparent gemacht werden, gewinnt der öffentliche Diskurs.

\subsubsection{Patienten mit besonderen Bedarfen}

Eine besondere Herausforderung für eine statusindifferente und diskriminierungsfreie Krankenhausversorgung in der Praxis stellen alle diejenigen Patientengruppen dar, die eines besonderen Aufwands in der Diagnostik, Therapie, Pflege, Begleitung und Kommunikation bedürfen und nicht selten auch spezielle Kenntnisse und Einfühlungsvermögen seitens der Behandelnden erfordern. Hierzu gehören neben Patienten mit seltenen Erkrankungen vor allem Kinder und Jugendliche, alte und multimorbide Patienten, Menschen mit Behinderung und Menschen mit Demenz sowie Patienten mit Migrationshintergrund und dadurch bedingten differierenden kulturellen Vorstellungen und eingeschränkten Sprachkenntnissen.

Grundsätzlich gilt, dass das Krankenhaus allen, die der stationären Krankenbehandlung bedürfen, offenstehen muss. Dennoch müssen zum einen diese Patientengruppen differenziert betrachtet und zum anderen die Maxime der wohnortnahen Versorgung mit dem Gebot der jeweils bestmöglichen Behandlung abgewogen werden. In der Stellungnahme der Zentralen Ethikkommission bei der Bundesärztekammer (ZEKO) zum Übereinkommen der Vereinten Nationen über die Rechte von Menschen mit Behinderungen (UNBehindertenrechtskonvention) heißt es beispielsweise, dass bei dem in der Konvention festgeschriebenen Grundsatz der gleichen Qualität und des gleichen Standards für die Gesundheitsversorgung von Menschen mit und ohne Behinderung „gleich“ nicht „identisch“ bedeute, „sondern eine dem individuellen Bedarf und der Lebenssituation von Menschen mit 
Behinderung angepasste medizinische Versorgung “. ${ }^{104}$ Die Konvention spricht den Menschen mit Behinderung aber auch die Gesundheitsleistungen zu, die von ihnen speziell wegen ihrer Behinderungen benötigt werden (Art. 25 b). ${ }^{105}$

Die Inklusion der unterschiedlichsten Patientengruppen in die regulären Behandlungsangebote der Krankenhäuser steht dabei in einem Spannungsverhältnis zum Aufbau spezialisierter Behandlungszentren, in denen die Fachexpertise konzentriert wird, deren Wohnortferne jedoch gerade für diese Patienten oft mit erheblichen sozialen und psychischen Belastungen verbunden ist. Spricht wegen der erforderlichen medizinischen Spezialkenntnisse bei der Behandlung seltener Erkrankungen sehr viel für die Konzentration auf wenige qualifizierte Zentren, kann für andere Gruppen wie Demenzbetroffene oder Menschen mit Behinderung, die einer Krankenhausbehandlung aus anderen Gründen als ihrer Demenz oder Behinderung bedürfen, die Perspektive einer wohnortnahen inklusiven Behandlung vorrangig sein.

\subsubsection{Bewusster Umgang mit Ressourcen}

Das Prinzip der Gerechtigkeit erfordert, mit knappen Ressourcen sorgfältig umzugehen. Vor diesem Hintergrund kommt der Ressourcenreflexivität ${ }^{106}$ als notwendigem Bestandteil des Handelns der Ärzte, Pflegenden und Therapeuten auch für die individuelle Patientenbehandlung eine besondere Bedeutung zu.

Im Rahmen seiner Stellungnahme „Nutzen und Kosten im Gesundheitswesen - Zur normativen Funktion ihrer

\footnotetext{
104 Zentrale Ethikkommission bei der Bundesärztekammer 2010, A298.

105 Gesetz zu dem Übereinkommen der Vereinten Nationen vom 13. Dezember 2006 über die Rechte von Menschen mit Behinderungen sowie zu dem Fakultativprotokoll vom 13. Dezember 2006 zum Übereinkommen der Vereinten Nationen über die Rechte von Menschen mit Behinderungen vom 21. Dezember 2008 (BGBI. II, 35).

106 Zum Begriff siehe Rixen et al. 2003, 193.
} 
Bewertung" hat der Deutsche Ethikrat den Nutzen und Schaden des Einsatzes von Instrumentarien untersucht, die die gesundheitsbezogene Lebensqualität messen. ${ }^{107}$ Als unstreitig anerkennt er einen sinnvollen Einsatz solcher Instrumentarien, wenn zwei konkurrierende medizinische Interventionen auf die Effektivität für ein und denselben Patienten hin überprüft werden sollen. Für solche Bewertungen sind nicht nur einzelne medizinische Therapien, sondern auch die Vorhaltung bestimmter Apparaturen oder Strukturen offen. Dem Ethikrat erschien es dagegen nicht akzeptabel, diese Instrumentarien zu nutzen, um Patienten oder Patientengruppen miteinander zu vergleichen und damit gegeneinander auszuspielen. Eine solche Verwendung würde Personen unter dem Gesichtspunkt ihrer „Kostenträchtigkeit“ gleichsam gegeneinander verrechnen und sie damit ethisch und verfassungsrechtlich unstatthaft allein als Objekte eines Kalküls behandeln. Die Analyse des Ethikrates hat darüber hinaus aufgezeigt, wie abhängig von oft nur implizit transportierten Wertentscheidungen solche Berechnungen sind: Ist es zum Beispiel legitim, die gewonnene Lebenszeit eines älteren Menschen als geringer zu bewerten als die eines jungen, wie es zum Teil geschieht?

Unabhängig von solchen Überlegungen gilt, dass Allokationsentscheidungen nicht einfach von der Makro- über die Mesoebene „nach unten“ weitergereicht und regelhaft dem einzelnen Krankenhaus oder gar dem individuellen Arzt aufgebürdet werden dürfen, der dadurch in eine mit den berufsethischen Forderungen unvereinbare Zwangslage geraten kann. Er müsste aufgrund der Begrenztheit medizinischer Ressourcen, die auf der Mikroebene zur Verfügung stehen, eine Auswahl zwischen seinen Patienten treffen und dem einen eine aussichtsreiche Behandlung vorenthalten, um sie einem anderen anbieten zu können.

Vor solchen Entwicklungen warnt auch die ZEKO. Sie unterscheidet zwischen Wirtschaftlichkeit und Ökonomisierung.

107 Deutscher Ethikrat 2011. 
Wirtschaftlichkeit wird als eine möglichst effiziente (sparsame) und effektive (wirksame) Allokation von Gütern und Dienstleistungen aufgefasst, und damit als ein für das ärztliche Handeln wichtiger Orientierungspunkt, „der als solcher zur moralischen Identität des Arztberufes keineswegs im Widerspruch“ stehe. Eine Ökonomisierung liege dagegen vor, „wenn betriebswirtschaftliche Parameter jenseits ihrer Dienstfunktion für die Verwirklichung originär medizinischer Aufgaben eine zunehmende Definitionsmacht über individuelle und institutionelle Handlungsziele gewinnen“. Gegen eine solche „ökonomische Überformung medizinischer Vollzüge“ wendet sich die Stellungnahme der ZEKO. ${ }^{108}$

Umgekehrt dürfen wirtschaftliche Überlegungen auch nicht zur Mengenausweitung ohne hinreichende Indikation führen. ${ }^{109}$ Diesbezüglich besteht die Befürchtung, dass in Abhängigkeit von der jeweiligen Vergütungssituation bestimmte Behandlungsarten verstärkt durchgeführt oder unterlassen werden. Ob allerdings Phänomene wie die Mengenausweitungen im Krankenhaus immer als direkte Folge der DRG-Einführung nachweisbar sind, ist umstritten. ${ }^{110}$

Aus den vorstehend umrissenen Überlegungen folgen organisationsstrukturelle Konsequenzen. Sowohl unter Effektivitäts- als auch Effizienzüberlegungen erscheint es angebracht, Strukturen institutionalisierter Verantwortungsübernahme zu

108 Zentrale Ethikkommission bei der Bundesärztekammer 2013a, A1753 f.

109 Ein Beispiel für die Schwierigkeiten, solche Befürchtungen zu verifizieren, ist die Kontroverse über die erst seit 2007 zur Verfügung stehende Methode der Transcatheter Aortic Valve Implantation (TAVI), des minimal-invasiven Aortenklappenersatzes am schlagenden Herzen bei älteren Risikopatienten. Diese Methode ist sehr viel teurer als die herkömmliche Operation am offenen Herzen, allerdings aufgrund ihrer Minimalinvasivität auch insbesondere bei älteren Patienten einsetzbar, die dem Risiko einer Operation nicht mehr ausgesetzt werden könnten. Umstritten ist die Strenge der Indikation, weil der Einsatz in den letzten Jahren enorm zugenommen hat (geschätzt wird, dass etwa 12.000 TAVIs von weltweit 40.000 in Deutschland durchgeführt wurden). Untersuchungen ergeben eine höhere postmortale Sterblichkeit nach der Operation als bei der herkömmlichen Operation, was die Befürworter allerdings mit den höheren Krankheitsbelastungen der Patienten erklären. Vgl. Gotzmann et al. 2011; sowie Tamburino et al. 2011.

110 Schreyögg et al. 2014 . 
stärken. Für die Makroallokationsebene gilt, dass hier institutionell identifizierbar alle grundlegenden Weichenstellungen politisch verantwortet werden müssen. Auf der Meso- und Mikroebene sind Risikomanagementsysteme ${ }^{111} \mathrm{zu}$ verknüpfen mit Qualitätssicherungsstrukturen, die es ermöglichen, Fehlentwicklungen systematisch zu identifizieren, als potenzielle Versorgungsrisiken zu erkennen und präventiv aufzufangen ${ }^{112}$ und auf diese Weise für die Implementierung von Ressourcenreflexivität zu sorgen.

Zusammenfassend lässt sich feststellen: Gerechtigkeit im Sinne von gleichem Zugang zu und gerechter Verteilung von Krankenhausleistungen umfasst sowohl das Gebot der statusindifferenten Gleichbehandlung (equality) als auch das Gebot des fairen und jeweils individuell patientenorientierten Einsatzes von Ressourcen (equity). Auch wenn die grundlegenden Allokationsentscheidungen auf den jeweils dafür zuständigen politischen und übergeordneten Ebenen zu fällen sind, müssen auch auf der Mikroebene, das heißt durch das Handeln des einzelnen Arztes und der einzelnen Pflegekraft, wirtschaftliche Überlegungen berücksichtigt werden. Gleicher Zugang und gerechte Verteilung bedeutet aufgrund der gegebenen Knappheit der Mittel und der Einsicht, dass Ressourcenindifferenz an der einen Stelle zum Schaden von Patienten an der anderen Stelle führen kann. Daraus ergibt sich das Gebot des ressourcenreflexiven Verhaltens aller Beteiligten, demzufolge Ressourcen im Krankenhaus stets effektiv und effizient einzusetzen sind. Allerdings schließt dieses Gebot eine primär ökonomische Ausrichtung ärztlichen wie pflegerischen Handelns aus, wie sie zum Beispiel gegeben wäre, wenn der Ressourceneinsatz zwischen verschiedenen Patientengruppen

111 Risikomanagement im Krankenhaus nimmt gesetzliche Anforderungen, wirtschaftliche Ziele, Qualitätsstandards und das Patientenwohl gleichermaßen in den Blick, um Risiken frühzeitig zu identifizieren und Maßnahmen zur Gegensteuerung einzuleiten oder eingetretene Fehler oder Schäden zu regulieren. Dabei wird weniger gefragt, wer schuld an einem Fehler war, sondern wodurch der Fehler aufgetreten ist.

112 Siehe dazu Höfling 2008, 32. 
verglichen würde, mit der Konsequenz, bestimmte Patienten oder Patientengruppen von einer erforderlichen Behandlung auszuschließen. 


\section{GEFÄHRDUNGEN DES PATIENTENWOHLS: KONFLIKTFELDER}

Mit dem Zielhorizont einer „guten“ Behandlung von Patienten im stationären Versorgungssystem verbinden Patienten, die im Krankenhaus tätigen Berufsgruppen und die Institution Krankenhaus aus ihrer jeweils eigenen Perspektive jeweils unterschiedliche Interessenschwerpunkte. Auch wenn alle diese Akteure die Interessen der jeweils anderen grundsätzlich als berechtigt, wünschenswert oder auch unabdingbar für eine angemessene Behandlung akzeptieren, steht in der Regel für Patienten eine Behandlung unter Wahrung ihrer Würde und personalen Selbstbestimmung im Vordergrund, für die medizinischen Berufsgruppen die Effektivität ihres professionellen Bemühens im Sinne eines bestmöglichen Behandlungsergebnisses, für die Ökonomie die Effizienz der Behandlung im Sinne eines günstigen Kosten-Nutzen-Verhältnisses und für das Krankenhaus die ökonomische Sicherstellung der eigenen Existenz oder sogar ein Gewinn. Diese unterschiedlichen Interessen treffen am Behandlungsort Krankenhaus zusammen und müssen hier vermittelt werden. Dabei steht das Krankenhaus als Mesoebene in einer komplexen Abhängigkeit von den als Mikroebene beschreibbaren Leistungserbringern der verschiedenen Berufsgruppen sowie den als Makroebene bezeichneten gesellschaftlichen Strukturen und politischen, gesetzlichen und ökonomischen Voraussetzungen und Rahmenbedingungen.

Versteht man das Patientenwohl als ethisches Leitprinzip einer "guten“ Behandlung, deuten zahlreiche Entwicklungen darauf hin, dass das stationäre Versorgungssystem in Deutschland zunehmend hinter diesem Anspruch zurückbleibt. Im Vordergrund von patientenbezogenen Entscheidungen stehen gegenwärtig als Outcome-Parameter vor allem die Effektivität und Effizienz der Behandlung, während andere für das Patientenwohl relevante Aspekte, wie zum Beispiel die Sorge für 
den Patienten oder der Respekt vor seiner Selbstbestimmung, nicht angemessen beachtet werden. Diese Faktoren sind ökonomisch nur schwer operationalisierbar und können einer vorrangig ökonomischen Sichtweise entgegenstehen. Insbesondere für Patientengruppen mit besonderen Bedarfen treten zudem die Aspekte eines gleichen Zugangs zu Behandlungsleistungen oder der gerechten Verteilung von Ressourcen hinter Kriterien der Effizienz und Effektivität zurück.

Eine zunehmende Vorrangstellung der Ökonomie bei Behandlungsentscheidungen wird unter dem Begriff der Ökonomisierung der stationären Versorgung diskutiert. Sie kann auf verschiedenen Ebenen des Systems zu ethischen Konflikten führen. So beklagen die im Krankenhaus Tätigen, dass die gegenwärtig für Allokationsentscheidungen herangezogenen Parameter die jeweiligen berufsethischen Anforderungen für eine am Patientenwohl ausgerichtete Behandlung nicht hinreichend berücksichtigen, sodass diese kaum mehr zu erfüllen sind. ${ }^{113}$ Andere Folgen betreffen den gleichen Zugang zur Krankenhausbehandlung von Patienten, die eines hohen Versorgungsaufwands bedürfen. Mit ethisch relevanten Folgen ist auch die Verzögerung oder ein Aussetzen von Investitionen durch die Krankenhäuser verbunden, wenn etwa der Staat seine mit dem dualen System der Krankenhausfinanzierung eingegangenen Verpflichtungen nicht hinreichend erfüllt. Zudem ergibt sich die ethische Frage, wieweit das Krankenhaus als soziale Institution und Nutznießer eines Solidarsystems seinerseits Gewinnbestrebungen jenseits der Deckung tatsächlich entstehender Kosten und sinnvoller Investitionen zurückstellen muss.

Im Folgenden werden Konfliktfelder dargestellt, die sich auf die drei Kriterien des Patientenwohls selbstbestimmungsermöglichende Sorge, hochwertige Behandlungsqualität sowie gleicher Zugang und gerechte Verteilung von Ressourcen beziehen.

113 Zentrale Ethikkommission bei der Bundesärztekammer $2013 a$. 


\subsection{Kommunikation}

Die Behandlungssituation im Krankenhaus und die damit verbundene Asymmetrie der Beziehung zwischen Behandler und Patient erfordert nicht nur den grundsätzlich geschuldeten Respekt vor der Selbstbestimmung des Patienten als Person, sondern zudem die sorgende Ermöglichung der Realisierung seiner Selbstbestimmung, wenn Letztere durch Faktoren, wie zum Beispiel Angst oder kognitive Einschränkungen, begrenzt oder erschwert ist. Auf die Möglichkeit, Selbstbestimmung zu realisieren, besteht ein berechtigter Anspruch des Patienten, der seinem Personsein entspringt. Dieser Anspruch wird nach einem modernen Medizinverständnis im Modell einer idealen Behandlungssituation aufgegriffen, in der die therapeutisch Handelnden gemeinsam mit dem Patienten ein auf ihn individuell zugeschnittenes Behandlungskonzept erarbeiten.

Es ist offensichtlich, dass ein am Patientenwohl orientierter Ansatz bestimmte Voraussetzungen erfordert. Hierzu gehören Zeit, Expertise und praktische Kommunikationskompetenz der im Krankenhaus tätigen Personen. ${ }^{114}$ Kommunikative Kompetenz setzt nicht nur linguistische Fähigkeiten einschließlich der Fähigkeit zu leichter Sprache voraus, sondern auch soziale Kompetenz, wie die Einfühlung in den Adressaten und die Bereitschaft, nonverbale Signale und Mitteilungen wahrzunehmen. Eine gelingende Kommunikation erfordert somit nicht nur entsprechende Kenntnisse und Fähigkeiten, sondern auch ausreichende persönliche Bereitschaft. Die Kommunikation im Krankenhaus wird darüber hinaus als ein hochsensibles Feld der Interaktion betrachtet, in dem sich Vorurteile, Vorbehalte und Diskriminierungspotenziale abbilden. Die Fähigkeit und Bereitschaft zu einer angemessenen Kommunikation steht daher gerade in der asymmetrischen Situation zwischen Behandler und Patient in enger Beziehung zu einem gerechten

114 Hoefert 2008, 167. 
Umgang mit dem Patienten und der Ermöglichung seiner Selbstbestimmung.

Angesichts des wachsenden Leistungsdrucks, des zunehmenden Zeitmangels und des Einsatzes der immer stärker den Alltag bestimmenden Informationstechnologien gewinnt neben der reinen Fachkompetenz die Kommunikationskompetenz der Beschäftigten eine immer größere Bedeutung. ${ }^{115}$ Neben der Kommunikation zwischen Arzt und Patient, Pflegekraft und Patient sowie Therapeut und Patient bezieht sich die geforderte Kompetenz auch auf die Kommunikation zwischen Vorgesetzten und Mitarbeitern und zwischen den Vertretern der einzelnen Berufsgruppen. Eine gute Kommunikation hat auch ökonomisch relevante Auswirkungen. Informationen, die in der Behandlung nicht berücksichtigt werden oder verloren gehen, können zu für alle Beteiligten belastenden (zum Beispiel unnötige oder doppelte Untersuchungen) bis hin zu schwerwiegenden (zum Beispiel Informationen über Unverträglichkeiten, Verwechslungen) Problemen führen. Der Kommunikationskompetenz im Krankenhaus kommt damit eine zentrale Rolle sowohl im Personal- und Organisationsmanagement als auch im Qualitätsmanagement zu. ${ }^{116}$ In diesem Zusammenhang ist auch auf die erforderliche Kommunikationskompetenz als Teil einer Managementkompetenz hinzuweisen, die Ärzte und Pflegende in Leitungspositionen benötigen. ${ }^{17}$

Kommunikation spielt auch bei interkulturellen Behandlungssituationen als Teil der „interkulturellen Kompetenz“ eine zentrale Rolle. „Interkulturelle Kompetenz" wird als Schlüsselkompetenz des 21. Jahrhunderts bezeichnet und folgendermaßen definiert: „Interkulturelle Kompetenz beschreibt die Kompetenz, auf Grundlage bestimmter Haltungen und Einstellungen sowie besonderer Handlungs- und

115 Klinkhammer/Krüger-Brand 2015.

116 Schaller/Baller 2008.

117 Seffner/Oberschelp 2013. 
Reflexionsfähigkeiten in interkulturellen Situationen effektiv und angemessen zu interagieren. "118 Für den Krankenhausalltag sind vor allem Fähigkeiten und Fertigkeiten wichtig, die in interkulturellen Behandlungssituationen eine ethisch angemessene Handlungsorientierung ermöglichen und eine Reflexion über diese Handlungen fördern. Dazu gehören unter anderem Kulturwissen, kultursensible Kommunikation, Vermeidung von Stereotypisierung sowie kritische Anerkennung und Selbstreflexion.

Um negative Auswirkungen mangelnder Kommunikation auf das Patientenwohl zu verhindern und die professionelle Selbstwahrnehmung zu verbessern, ist es erforderlich, dass der Erwerb von Kommunikationskompetenz und interkultureller Kompetenz zu einem festen Bestandteil der Aus-, Fort- und Weiterbildung in den Gesundheitsberufen wird. Zudem ist es notwendig, im Krankenhaus die strukturellen und prozeduralen Voraussetzungen für eine verlässliche und kultursensible Kommunikation zu schaffen, Letztere zum Beispiel in Form von medizinisch kundigen und mit dem deutschen Gesundheitssystem und seinen ethischen Grundlagen vertrauten Übersetzern sowie Seelsorgern. Für gehörlose Patienten ist dementsprechend eine Übersetzung in Gebärdensprache erforderlich.

\subsection{Berufsethos und Berufswirklichkeit}

Ein weiteres Konfliktfeld, das sich für die verschiedenen im Krankenhaus tätigen Berufsgruppen im Hinblick auf eine am Patientenwohl orientierte Behandlung ergibt, bezieht sich auf das jeweilige berufliche Ethos, die damit verbundenen Überzeugungen und Ziele und die Krankenhausrealität. Widersprüche zwischen Berufsethos und Berufswirklichkeit können als Indikator für Gefährdungen des Patientenwohls angesehen werden.

118 Bertelsmann Stiftung 2006, 5. 


\subsection{1 Ärztlicher Bereich}

Für Ärzte stellt der von Tom Beauchamps und James Childress entwickelte, weitverbreitete Ansatz der „vier Prinzipien der biomedizinischen Ethik“ einen wichtigen Orientierungsrahmen dar. Zwei dieser Prinzipien, das Prinzip des Wohltuns (beneficence) und des Nichtschadens (non-maleficence), sind schon seit Hippokrates Bestandteil des ärztlichen Ethos. Dagegen ist das Prinzip des Respekts vor der Autonomie des Patienten - zumindest in seiner heutigen Form und nach aktuellem Verständnis - relativ neu. Das Prinzip der Gerechtigkeit gilt zwar als ein klassischer Grundsatz der Ethik, wird jedoch von den Autoren dieses Ansatzes für den Bereich der medizinischen Versorgung durch eine neue Schwerpunktsetzung spezifiziert.

Das Prinzip der Autonomie bzw. des Respekts vor der Autonomie des Patienten richtet sich gegen eine Bevormundung des Patienten durch den Behandelnden und zielt auf die Ermöglichung einer informierten und freien Patientenentscheidung in der Behandlungssituation ab. Das Prinzip des Nichtschadens rekurriert auf den klassischen ärztlichen Grundsatz des primum nihil nocere. Es fordert die Vertretbarkeit der mit dem Eingriff verbundenen Risiken und Nebenwirkungen und verlangt damit hohe qualitative Standards und ihre individuelle Anpassung an die jeweilige Situation des Patienten. Das Prinzip des Wohltuns benennt die ärztliche Verpflichtung, Krankheiten zu vermeiden, zu heilen und das Leid des Patienten zu lindern. Es fordert damit den ärztlichen Grundsatz des salus aegroti suprema lex, der traditionell als das klassische Prinzip der Medizin schlechthin galt. Das Prinzip der Gerechtigkeit fokussiert auf die Gleichbehandlung der Patienten und einen gerechten Zugang zur Gesundheitsversorgung. Wenngleich die theoretische Grundlegung, das Verhältnis der vier Prinzipien untereinander und ihre Rolle und Tragweite bei der konkreten ethischen Entscheidungsfindung Gegenstand (hier nicht weiter behandelter) kontroverser Diskussionen sind, ist 
es ersichtlich, dass die vier Prinzipien ethische Pflichten aufseiten des Arztes einfordern, die teilweise Entsprechungen im Recht finden. So muss der Arzt den Patienten als Voraussetzung für dessen selbstbestimmte Entscheidung gewissenhaft und umfassend über geplante medizinische Maßnahmen und deren Nebenwirkungen und Risiken informieren, sich hierfür die erforderliche Zeit nehmen und gegebenenfalls abweichende Entscheidungen des Patienten respektieren. Die mit dem Eingriff verbundenen Risiken und Nebenwirkungen machen für eine Beachtung des Gebotes des Nichtschadens ein ärztliches Handeln notwendig, das sich auf hinreichende Kompetenz und Erfahrung abstützt, sowie in der Regel die Anwendung eines Verfahrens, das anerkannten Qualitätsstandards entspricht. Die Feststellung einer rechtfertigenden medizinischen Indikation in Beachtung des Prinzips des Wohltuns erfordert neben der medizinischen Expertise eine Beurteilung des individuellen Patienten und eine Abstimmung der medizinischen Maßnahmen auf seine individuelle Lebenssituation. Ein diskriminierungsfreier Zugang von Patienten zur ärztlichen Behandlung als Forderung des Gerechtigkeitsprinzips verlangt unter anderem die Bereitschaft, auch Patienten mit besonderem Zuwendungsbedarf nicht von der Behandlung fernzuhalten und die verfügbaren Ressourcen gerecht zu verteilen. Mit diesen Forderungen erweisen sich die vier Prinzipien als notwendige Bedingungen für die Sicherstellung des Patientenwohls.

Vor dem Hintergrund dieser Prinzipien als viel beachteter Grundlage der ärztlichen Ethik wird die gegenwärtige Situation der Patientenbehandlung im Krankenhaus von vielen dort arbeitenden Ärzten wegen der ökonomischen Rahmenbedingungen, die zu hohem Arbeitsdruck und Arbeitsverdichtung führen und hierdurch eine angemessene Patientenbehandlung in vielen Fällen erschweren, zunehmend als ethisch 
problematisch und mit dem beruflichen Selbstverständnis kaum vereinbar erlebt. ${ }^{19}$

Die beiden Normensysteme einer dem individuellen Patientenwohl verpflichteten medizinischen Ethik und einer durch ökonomische Kriterien charakterisierten Marktsituation sind nicht prinzipiell einander entgegengesetzt. Gleichwohl können ihre unterschiedlichen Primärorientierungen zu erheblichen Konflikten führen. Wettbewerb und eine ressourcenbewusste Versorgung können dem Patientenwohl durchaus dienlich sein, indem sie dazu beitragen, ärztliche Diagnostik und Therapie auf einen notwendigen und angemessenen Rahmen zu beschränken und etwa die Prozess-, Struktur- und Ergebnisqualität zu verbessern. Dies war auch eines der Ziele der Reform der Krankenhausfinanzierung, die zur Einführung der fallpauschalbasierten Vergütung (DRG) führte. Zudem gehört der verantwortungsvolle Umgang mit knappen Ressourcen zu den ethischen Pflichten des Arztes. ${ }^{120}$ Zwingt eine angespannte Marktsituation im stationären Versorgungssektor hingegen zu einem Konkurrenzkampf um beschränkte Ressourcen, sodass damit die Existenzfrage für ein Krankenhaus oder eine Fachabteilung in einem Krankenhaus verbunden ist, besteht die Gefahr, dass fremdnützige Aspekte, die eine patientenwohlorientierte Behandlung beeinträchtigen, in die Behandlungssituation und die Arzt-Patient-Beziehung einfließen. In diesem Fall geraten die Normsysteme einer dem Patientenwohl verpflichteten medizinischen Ethik und einem Handeln primär nach ökonomischen Grundsätzen in Konflikt.

Mit der Einführung der DRGs im stationären Versorgungsbereich wurde ein Abrechnungssystem in die Krankenhausversorgung eingeführt, das als Mittel für eine verbesserte

119 Für 81 Prozent der befragten Chefärzte war der wirtschaftliche Druck spürbar; 45 Prozent nahmen oft Entscheidungskonflikte zwischen ärztlichen und wirtschaftlichen Zielsetzungen wahr; 70 Prozent sahen sich durch wirtschaftliche Rahmenbedingungen in der Berufsausübung eingeschränkt (Reifferscheid/Pomorin/Wasem 2015, e132).

120 Woopen 2009. 
Nutzung von Ressourcen in der stationären Versorgung dienen sollte. So wurden lange Verweildauern von Patienten, die sich im vorherigen Abrechnungssystem nach tagesgleichen Pflegesätzen unter Umständen erlössteigernd auswirkten, durch eine Abrechnung nach DRGs im Prinzip für das Krankenhaus unwirtschaftlich. Beide Berechnungsprinzipien enthalten ethisch problematische Anreize im Hinblick auf das Patientenwohl, insofern im erstgenannten Fall eine unnötig lange Verweildauer von Patienten im Krankenhaus ökonomische Vorteile erwarten lässt, während im zweiten Fall eine medizinisch unangemessen kurze Verweildauer eines Patienten, die die anschließende Behandlung möglichst vieler weiterer Patienten und damit den Erlös weiterer Behandlungspauschalen in möglichst kurzer Zeit erlaubt, ökonomische Vorteile verspricht. Unter den mit den DRGs eingeführten Rahmenbedingungen besteht aus ökonomischer Perspektive die Gefahr, den Patienten nach der Diagnosestellung und diagnosebezogenen Behandlung zu früh aus dem Krankenhaus zu entlassen. Solche und andere Handlungsweisen wie zum Beispiel eine ausgeweitete Indikationsstellung oder eine Fragmentierung des Behandlungsprozesses legen die auf Basis empirischer Untersuchungen mittlerweile gut begründete These nahe, dass der Patient unter Bedingungen des derzeitigen DRG-Systems weniger in seiner individuellen Bedürftigkeit als vielmehr als ein pauschalierter Behandlungsfall wahrgenommen wird. Hiermit droht eine veränderte Arzt-Patient-Beziehung. Denn wenn die Fallpauschale die Sicht des Arztes auf den Patienten als Individuum überformt und ihre Funktion von einem Mittel für die Abrechnung hin zum Zweck der Behandlung verändert, erscheint das wichtige Vertrauensverhältnis zwischen Arzt und Patient ernsthaft gefährdet. ${ }^{121}$

Zugunsten welcher Seite der Konflikt zwischen Ökonomisierung und Selbstverständnis der Medizin aufgelöst werden

121 Vogd 2006; Vogd 2014, 58-73; Vogd 2015; Vogd 2016; Feißt/Molzberger 2016; Wolf/Ostermann 2016. 
kann und welche Folgen daraus für die Arbeitsbedingungen der Beschäftigten und die Patientenversorgung entstehen, wird in vergleichenden Studien höchst unterschiedlich bewertet. Dies hänge von Faktoren wie der gesamtwirtschaftlichen Situation des Hauses, der Einbindung der Mitarbeiter in die Organisationsentwicklung, der Qualitätssicherung und dem jeweiligen Leitbild des Hauses ab, sodass sich daraus die Unterschiedlichkeiten zwischen den verschiedenen Häusern erklären. ${ }^{122}$ Bestätigt wird aber in Studien, dass der Konflikt nicht nur auf der Ebene der unmittelbaren Patientenbehandlung angekommen ist, sondern sich auch mit der DRG-Einführung deutlich verschärft hat. ${ }^{23}$

Die skizzierte Ziel-Mittel-Verschiebung kann ethisch problematische Folgen haben, die sich insbesondere in folgenden, von vielen im Krankenhaus arbeitenden Ärzten beklagten Entwicklungen darstellen können:

» So wird häufig geäußert, dass Krankenhäuser aus je eigener, manchmal ökonomischer Perspektive ein Interesse daran hätten, Patienten so früh wie möglich aus dem Krankenhaus zu entlassen. Dieses Interesse transformiere sich nicht selten in einen beständigen Druck auf behandelnde Ärzte, die Entlassung eines Patienten aus der stationären Behandlung auf einen Zeitpunkt einer klinisch nicht konsolidierten Wiederherstellung vorzuverlegen. Auch könnten eine schwierige, aufwendige und langwierige differenzielle Diagnostik als auch Komplikationen, die die Entlassung des Patienten aus dem Krankenhaus verzögern, erheblichen wirtschaftlichen Druck auf das Krankenhaus erzeugen, der schließlich auf die behandelnden Ärzte ausgeübt werde. Kompensatorische Maßnahmen, wie zum Beispiel die kurzzeitige Entlassung und Wiederaufnahme von $\mathrm{Pa}$ tienten oder ihre Verlegung in andere Krankenhäuser mit

122 Buhr/Klinke 2006.

123 Vgl. hierzu unter anderem Marckmann/Strech 2009. 
einer bereits eingeplanten Rückübernahme zu einem späteren Zeitpunkt unter einer anderen Diagnose (sogenanntes Patientenkarussell bzw. Drehtüreffekt), dienten zumeist nicht dem Wohl des Patienten und einer gesamtökonomisch sinnvollen und qualitativ hochwertigen Behandlung. Hingegen bestehe bei Komplexpauschalen, bei denen verschiedene Behandlungsmaßnahmen in eine Fallpauschale zusammengefasst sind, die Gefahr, dass Patienten bis zur vollständigen Erfüllung der Pauschale in der Einrichtung behandelt würden, auch wenn diese Maßnahmen aus medizinischer Sicht nicht unbedingt erforderlich seien.

» Es bestehe ein ökonomisch bedingtes Interesse, vorwiegend Patienten mit bestimmten umschriebenen Krankheitsbildern zu behandeln und die Aufnahme von Patienten mit komplikationsbehafteten Erkrankungen zu vermeiden (Selektion der Patientenaufnahme). Dieses Problem manifestiere sich zum Beispiel in der Ablehnung oder Verzögerung der stationären Aufnahme von Patienten, die als zusätzlichen klinischen Befund ein demenzielles Syndrom, Bettlägerigkeit, Isolationspflicht bei multiresistenten Keimen o. Ä. aufweisen und daher einer personal- und kostenintensiven Betreuung bedürfen.

» Beklagt wird, dass sich infolge der Verkürzung der Verweildauern der Patienten der Patientendurchsatz durch das Krankenhaus und damit die Arbeitsintensität und in der Folge die zeitliche Belastung für Ärzte (Arbeitsverdichtung) erheblich erhöhe, worauf die Personalstrukturen nur teilweise abgestimmt seien. Sowohl durch kurze Verweildauern als auch durch höheren Patientendurchsatz stehe dann für den Kontakt des Arztes zum Patienten weniger Zeit zur Verfügung, wodurch eine Behandlung, bei der 
individuelle Besonderheiten berücksichtigt werden, deutlich erschwert werde. ${ }^{124}$

\D Durch seine Fokussierung auf erbrachte Leistungen begünstige das Abrechnungssystem der DRGs möglichst weitgehend standardisierbare Eingriffe und folglich eine Spezialisierung auf solche Behandlungen. Damit sei auch ein Anreiz verbunden, die medizinische Indikation für solche Eingriffe zunehmend weit zu stellen. Hierdurch lasse sich nach Meinung vieler das Problem der Ausweitung der Behandlungszahlen („Mengenausweitungen“) erklären, das für bestimmte Arten von Eingriffen vor allem seit der Einführung der DRGs zu beobachten ist. ${ }^{125}$ Hinzu komme, dass bestimmte medizinische Fachbereiche, wie zum Beispiel die Geburtshilfe oder Kinderheilkunde, für Krankenhäuser ökonomisch nicht attraktiv sind und folglich abgebaut würden. Mit einer solchen mittelbar systembedingten Erschwerung des Zugangs bestimmter Patientengruppen zu spezialisierter Behandlung könne ein diskriminierendes Moment verbunden sein.

» Trotz höherer Standardisierung vieler Behandlungen und größerer Möglichkeiten von Patienten, sich über Gesundheitsfragen in Eigeninitiative zu informieren, nehme der Kommunikationsbedarf zwischen Arzt und Patient nicht etwa ab, sondern deutlich zu. Grund hierfür seien häufig Missverständnisse oder Fehlinformationen aufseiten des Patienten, deren Berichtigung erheblichen zeitlichen und kommunikativen Aufwand erfordert. Die Kommunikation mit dem Patienten habe dabei zunehmend die Aufgabe, patientenspezifische Behandlungsbefunde und sein

12472 Prozent der befragten Chefärzte vertreten die Meinung, dass ausreichend Zeit für die Zuwendung zum Patienten nur „selten“ oder „manchmal“ vorhanden ist (Reifferscheid/Pomorin/Wasem 2014, 5).

12539 Prozent der befragten Chefärzte glaubten, dass in ihrem Fachgebiet wirtschaftliche Rahmenbedingungen zu überhöhten Fallzahlen führen (Reifferscheid/Pomorin/Wasem 2015, e129, aufgeschlüsselt nach Fachgebieten in Abb. 2 [e134]). Ein populäres Beispiel für Mengenausweitung ist die Linksherzkatheter-Untersuchung. Vgl. auch Schreyögg et al. 2014, 13. 
medizinisches Vorverständnis in angemessene Kongruenz $\mathrm{zu}$ bringen. Solche Information und Kommunikation seien wesentliche Bedingungen für eine selbstbestimmungsermöglichende Sorge für den Patienten. Die Zeit, die den Ärzten für diese Aufgaben im Krankenhaus zur Verfügung steht, reiche nach allgemeinem Dafürhalten längst nicht mehr aus und verringere sich zudem ständig weiter.

»Als problematisch werden auch die zunehmend umfangreichen Dokumentationspflichten im ärztlichen Bereich angesehen, deren Detailliertheit allein mit dem Wohl der Patienten nicht mehr zu begründen sei. Dabei wird nicht die Pflicht der Dokumentation als solche kritisiert, sondern ein auf vorwiegend ökonomische Gründe zurückzuführender Aufwand an Dokumentation und Zeit, die für die eigentliche Behandlung des Patienten nicht mehr zur Verfügung stehen. Hierdurch werde die Entwicklung von einer „sprechenden“ zu einer „dokumentierenden“ Medizin gefördert.

» Als Folgeprobleme lasse sich beobachten, dass junge, ins Berufsleben startende Ärzte die skizzierte Situation als ethischen Standard der deutschen Krankenhausmedizin verinnerlichten. Ethische Rückfragen würden oftmals aufgrund der Dichte des Arbeitsalltags und aufgrund hierarchischer Abhängigkeiten nicht vorgebracht oder nicht beantwortet. Zudem könnten Fachabteilungen in Krankenhäusern aufgrund der angespannten Personalsituation im ärztlichen Bereich eine organisierte und strukturierte Weiterbildung und Supervision junger Ärzte kaum noch durchführen, weshalb jungen Ärzten oftmals Aufgaben übertragen würden (oder übertragen werden müssten), auf die sie nicht ausreichend vorbereitet sind. Dem Ärztemangel im stationären Versorgungsbereich werde gegenwärtig unter anderem durch die verstärkte Anwerbung von Ärzten aus dem Ausland begegnet, deren Einsatz sich allerdings gerade in einem kommunikations- und kultursensiblen Bereich wie dem Krankenhaus schwierig gestalten könne. Überdies 
fehlten diese Ärzte in der Gesundheitsversorgung in ihren Herkunftsländern.

Vor diesem Hintergrund ist zu fragen, wie die Erfordernisse eines wirtschaftlich sinnvollen und ressourcenschonenden Handelns mit den Maximen einer Orientierung am individuellen Patientenwohl in Einklang gebracht werden können. Eine Priorisierung der einen oder der anderen Seite führt jeweils zu risikoreichen Zuspitzungen, die entweder die Qualität der Versorgung oder die wirtschaftliche Überlebensfähigkeit eines Krankenhauses gefährden können. Lösungsansätze können darin bestehen, die Berechtigung der verschiedenen Interessen sichtbar zu machen und eine Praxis des transparenten, das heißt nach akzeptierten Regeln und Kriterien gestalteten, immer wieder neuen Aushandelns des Interessensausgleichs im Einzelfalls herbeizuführen. Dies erfordert neben einer hohen kommunikativen Kompetenz geeignete Strukturen und zeitliche Ressourcen. Die im Rahmen der innerbetrieblichen Budgetierung schon heute eröffneten Gestaltungsspielräume für einzelne Fachabteilungen müssen ausreichend finanziert sein. Erforderliche Begrenzungen müssen plausibel und transparent begründet sein.

Für die Ärzteschaft ist es in diesem Zusammenhang von erheblicher Bedeutung, sich in einem strukturierten Prozess der ethischen Grundlagen ihres Berufes vergewissern zu können. Ein Ziel muss die Sicherstellung eines auf den individuellen Patienten und seinen spezifischen Bedarf abstellenden ärztlichen Handelns sein. Hieraus abgeleitet ergibt sich als ein weiteres Ziel, die Qualität der medizinischen Behandlung auch unter ethischer Perspektive angemessen $\mathrm{zu}$ definieren und die hierfür relevanten Kriterien zu benennen. Zudem sollten Krankenhausärzte ihre bestehenden Organisationsstrukturen nutzen, um auch die ethischen Grundlagen ihres Berufes wirksam in (gesundheits)politische Entscheidungsprozesse über den stationären Versorgungsbereich einzubringen und damit die Systemebene verantwortlich mitzugestalten. 


\subsubsection{Pflegerischer Bereich}

Wie im ärztlichen Bereich gibt es auch im Bereich der Pflege eine Bereichsethik im Sinne der Reflexion und Beurteilung moralischer Fragen und Probleme der Pflegepraxis, die sich als Pflegeethik versteht. ${ }^{126}$ In Parallele zur Unterscheidung der „Medizinethik“ von einer „Ethik in der Medizin“ wird neben der „Pflegeethik“ auch von einer „Ethik in der Pflege“ gesprochen, wobei unklar bleibt, ob und in welchem Sinn sich beide Begriffe unterscheiden. Unterschiedliche Auffassungen bestehen darüber, inwieweit Pflegeethik eine spezifische Berufsethik darstellt, da eine erhebliche Zahl von Pflegeleistungen außerhalb der Pflegeprofession stattfinden (die pflegebedürftigen Menschen werden mehrheitlich von Angehörigen gepflegt) und sich Pflegeethik sowohl auf die berufliche als auch die nicht berufliche Pflegepraxis beziehen lässt. ${ }^{127}$ Auch die inhaltlichen Aspekte einer Pflegeethik als eigene Bereichsethik und die Frage nach einer Abgrenzung gegenüber einer als ärztliche Ethik verstandenen Medizinethik werden unterschiedlich eingeschätzt. ${ }^{128}$ Eine Rechtfertigung für eine eigene Pflegeethik wird vor allem darin gesehen, dass die Pflege eine eigene Perspektive auf den Patienten und auf ethische Fragen im Gesundheitswesen besitzt. Pflegende gewärtigen durch die Eigenart der Pflegetätigkeit spezifische, ethisch relevante Fragestellungen und Probleme. Daher wird argumentiert, dass Pflegende ihre eigene Praxis eigenständig ethisch reflektieren und an allen wichtigen patientenbezogenen Entscheidungen beteiligt werden müssen, nicht zuletzt auch deshalb, weil sie oft genauer über die Lebenssituation und die Wünsche der Patienten Bescheid wissen als die Ärzte. ${ }^{129}$ Die Forderung nach Etablierung einer Pflegeethik als Bereichsethik verbindet sich zudem mit der Forderung nach mehr beruflicher Autonomie

$126 \mathrm{Vgl}$. unter anderem Heffels 2002, $65 \mathrm{ff}$.

127 Lay 2012, 110.

128 Georg/Frowein 1999, 683.

129 Rabe 2009, $68 \mathrm{ff}$. 
für die Pflege als Voraussetzung für moralisches Handeln, so wie umgekehrt die Etablierung einer Pflegeethik verschiedentlich als Professionalisierungsmerkmal der Pflege verstanden wird. Nach anderer Auffassung teilen Medizin und Pflege ein gemeinsames Handlungsfeld, auf das sich auch der gesellschaftliche Auftrag bezieht und dem eine starke Unterscheidung zwischen einer Pflegeethik und der Medizinethik nicht gerecht wird, auch wenn sich im Zuge der Differenzierung und Professionalisierung die spezifischen Handlungsfelder der einzelnen Berufe zunehmend deutlich voneinander absetzen. ${ }^{30}$ Eine tugendethisch orientierte Auflistung von Handlungsund Haltungsmaximen findet sich unter anderem in Ansätzen der Care-Ethik. Pflegende sollten danach in besonderem Maß über attentiveness (Aufmerksamkeit), responsibility (Verantwortlichkeit), competence (Kompetenz), responsiveness (Antwortfähigkeit) und mindfullness (Achtsamkeit) verfügen. ${ }^{31}$ Solche professionsethischen Pflichten verstehen sich als angestrebte Haltungen, die mit der eigenen professionellen und persönlichen Entwicklung gemäß dem heute in der Pflegewissenschaft eingeführten Modell der stufenweisen Kompetenzentwicklung erworben werden. ${ }^{132}$

Aus Sicht der Patienten stellen die Pflegenden im Krankenhaus eine für die erlebte Behandlungs- und Umgangsqualität sehr wichtige Berufsgruppe dar. Dementsprechend spielen Zeitnot der Pflegenden, häufiger Personalwechsel und das Ersetzen ausgebildeter Kräfte durch Hilfspersonal gerade für körpernahe Verrichtungen bei einer negativen Bewertung von Krankenhausleistungen durch Patienten, aber auch in der Selbsteinschätzung der Pflegenden eine herausragende Rolle.

Personalabbau im Pflegebereich, Verweildauersenkung und Fallzahlsteigerung führen auch in der Pflege $\mathrm{zu}$ einer erheblichen Arbeitsverdichtung. ${ }^{33}$ Hierzu trägt auch die

130 Ebd., $70 \mathrm{ff}$.

131 Vgl. unter anderem Tronto 1993.

132 Benner 2000.

133 Deutsches Institut für angewandte Pflegeforschung 2014. 
Übertragung von weniger anspruchsvollen Tätigkeiten, die bisher zum Kernbestand des Pflegeberufes gezählt wurden, an weniger qualifizierte Hilfskräfte bei, wodurch sich aufgrund des Wegfalls weniger intensiver Arbeitsphasen die Leistungen im eigentlichen Pflegeberuf zusätzlich verdichten. ${ }^{134}$ Ein permanenter Zeitdruck ist für viele Pflegende heute zur Norm geworden und wirkt sich auf die Pflegeleistung aus. ${ }^{135}$ Dies betrifft zunächst die „unsichtbaren“ Arbeiten wie Zuhören, Trostspenden, Für-Wohlbefinden-Sorgen, des Weiteren auch prophylaktische Maßnahmen zur Verhinderung von Komplikationen (Dekubitus, Thrombose, Pneumonie, Kontrakturen), ferner auch Aktivitäten der Grundpflege, die entindividualisiert werden, sowie überdies das Informieren, das Beantworten von Fragen und die Kommunikation über das Verhalten nach der Entlassung aus dem Krankenhaus. Konzepte wie die der Bezugspflege oder des primary nursing, die auf jeweils festen Zuordnungen von Pflegenden zu Patienten aufbauen, haben damit in der Krankenhauspraxis heute praktisch keinen Raum mehr. Das gegenwärtige System der stationären Versorgung in Deutschland scheint diese Entwicklungen zu begünstigen. So gibt es empirische Hinweise, dass die implizite Rationierung ${ }^{136}$ von Pflegeleistungen in Deutschland ${ }^{137}$ im Vergleich mit elf weiteren europäischen Ländern besonders ausgeprägt ist. ${ }^{138}$ Bekannt ist auch, dass vielfach Mitarbeiter häuslicher Pflegedienste und in der stationären Altenpflege über den schlechten

134 Vgl. Friesacher 2015.

135 Heiner Friesacher spricht hier auch vom „unerträglichen Begleiter“ der Pflegearbeit (persönliche Kommunikation).

136 Unter Rationierung im Gesundheitswesen wird die begrenzende Zuteilung von Ressourcen nach entweder offen gelegten (expliziten) oder nicht offen gelegten (impliziten) Kriterien verstanden (Deutscher Ethikrat 2011, 22). Vgl. hierzu auch Fuchs/Nagel/Raspe 2009, A555 f.

137 Nur 21 Prozent der Pflegedienstleitungen sind der Meinung, dass das Pflegepersonal sämtliche erforderliche Pflegeleistungen immer durchführen kann, 70 Prozent denken, dass das „eher nicht“ der Fall ist und 9 Prozent sehen eine ausreichende Versorgung als „überhaupt nicht“ gewährleistet an (Reifferscheid/Pomorin/Wasem 2014, 6). 72 Prozent berichten von häufigen Entscheidungskonflikten in der Abwägung zwischen Wirtschaftlichkeit und pflegerischen Zielen (Reifferscheid/Pomorin/Wasem 2014, 4).

138 Zander et al. 2014; Ausserhofer et al. 2014. 
Pflegezustand ihrer Patienten nach Entlassung aus dem Krankenhaus klagen. ${ }^{139}$ Bei kognitiv eingeschränkten Patienten ist nach einem stationären Aufenthalt oft der vorher noch erhaltene Teil lebenspraktischer Selbstständigkeit nicht mehr vorhanden, weil aus Zeitdruck ersetzende Pflege statt einer die Selbstständigkeit unterstützenden Pflege geleistet wurde.

Eine weitere sichtbare Veränderung ist die stärkere Steuerung der Pflegearbeit durch die Vernetzung der klinischen Daten mit betriebswirtschaftlichen Instrumenten. Ein Beispiel hierfür ist das Scoring-Verfahren TISS (Therapeutic Intervention Scoring System) ${ }^{140}$, mit dem sich medizinisch-technische Maßnahmen deutlich besser abbilden lassen als zeitaufwendige Pflegearbeit (zum Beispiel einen Patienten in den Arm zu nehmen, Gespräche zu führen, ihm die Angst zu nehmen, mit eventueller Luftnot umzugehen) und damit die Zurückdrängung der Pflege im Sinne einer auf den ganzen Patienten ausgerichteten Hilfe und Sorgemaßnahme zugunsten technischer Anwendungen und Prozeduren beschleunigt.

Eine besondere Rolle spielt auch der demografisch bedingt rasant wachsende Pflegebedarf, auf den die Krankenhäuser nur unzureichend vorbereitet sind. Entgegen dem absehbaren Bedarf werden nicht nur Personalstellen im Pflegebereich abgebaut, sondern Pflegeleistungen auch durch Personalsplitting (Einsatz von Auszubildenden oder angelernten Hilfskräften) riskant dequalifiziert.

Erschwerend kommt hinzu, dass sich die Berufsgruppe der Pflegenden zunehmend nur schwer selbst vertreten kann.

139 Persönliche Kommunikation durch den Pflegewissenschaftler Heiner Friesacher.

140 TISS ist ein Scoring-System zur Quantifizierung des Pflegeaufwandes von schwerkranken Patienten, das 1974 entwickelt wurde. Bewertet werden dabei Maßnahmen wie zum Beispiel Monitoring, Verabreichung von Medikamenten, Verbandswechsel, mechanische Beatmung, Katheterisierung oder parenterale Ernährung. Psychosoziale Maßnahmen wie Beruhigen, Sprechen oder Angstnehmen werden nicht erfasst. Seit seiner Einführung hat er sich weit verbreitet und gilt mittlerweile als etablierter StandardScore. Nach mehreren Modifikationen wurde das System 1996 von den ursprünglichen 76 zu bewertenden Maßnahmen auf 28 reduziert (daher nunmehr TISS-28). 
Pflegende sind - obwohl zahlenmäßig die größte Berufsgruppe im Krankenhaus - am wenigsten in den Leitungen vertreten. Auch auf übergeordneter Ebene werden pflegerelevante Entwicklungen und Standards von anderen Interessengruppen (Spitzenverband der Krankenkassen, Deutsche Krankenhausgesellschaft, Bundesärztekammer) dominiert und zur Kostensenkung oder Gewinnmaximierung reduziert bzw. blockiert.

Alle diese Entwicklungen führen dazu, dass die Pflegenden ihren ethischen Grundsätzen, die von einer Sorge für den Patienten und Achtsamkeit ihm gegenüber geprägt sind, zunehmend nicht mehr gerecht werden können. Hiervon werden auch pflegerische Grundhaltungen berührt, die die Praxis der Pflege in einer stark reduktionistischen Auffassung von „Dienstleistung“ eher an dem Modell der Erbringung definierbarer Leistungen für einen Kunden orientieren, während der Aspekt der mitmenschlichen und selbstbestimmungsfördernden Zuwendung von der Verfügbarkeit gegebenenfalls residualer zeitlicher Ressourcen abhängig gemacht wird. ${ }^{141} \mathrm{Zu}$ dieser Tendenz trägt bei, dass der Kernbereich pflegerischer Tätigkeit zunehmend nicht mehr aus der Pflege selbst, sondern aus ökonomischer Perspektive definiert wird, indem vermeintlich anspruchslose Tätigkeiten, die jedoch für das Selbstverständnis und die Ergebnisse der ganzheitlichen Pflege einer Person von erheblicher Bedeutung sind, an kostengünstigere Hilfskräfte vergeben werden. Festzustellen ist, dass gerade die von der Pflege entwickelten prozess- und kooperationsorientierten Ansätze für eine Neuorientierung im Krankenhaus von besonderer Bedeutung sind.

Vor diesem Hintergrund ist es erforderlich, die Bedeutung der Pflege für eine identitätsstiftende Entwicklung des Krankenhauses neu zu bewerten und der Pflege insgesamt eine höhere Wertschätzung zukommen zu lassen. Bei aller gebotenen

14188 Prozent der Pflegedienstleitungen vertreten die Meinung, dass ausreichend Zeit für die Zuwendung zum Patienten nur „selten“ oder „manchmal“ vorhanden ist (Reifferscheid/Pomorin/Wasem 2014, 5). 
Vorsicht, da eine Auswertung der vorhandenen Innovationsprojekte zur Verbesserung der Pflegesituation im Krankenhaus 2011 keine eindeutigen Ergebnisse erbracht hat ${ }^{142}$, sollten im Interesse einer höheren Bewertung der Pflege im Krankenhaus neben einem zahlenmäßigen Zuwachs der Personalstellen insbesondere berücksichtigt werden:

» die Gewährleistung des selbstbestimmungsermöglichenden Umgangs mit Patienten in der Grundpflege sowie in der Behandlungspflege, wofür entsprechende fachliche Expertise, ausreichende Zeitbudgets für den individuellen Patientenkontakt, Einplanung größerer Zeitkorridore für Übergaben, Sicherstellung interprofessioneller Visiten, ein ausreichender kollegialer Austausch sowie die Durchführung von Fallkonferenzen notwendig sind,

» eine größere Beteiligung der Pflegenden an Steuerungsprozessen und Leitungsentscheidungen im Krankenhaus (Krankenhausleitung, Leitung von Fachabteilungen),

» qualifizierte Pflegeexperten auf der Ebene der Direktorien oder der Abteilungsleitungen sowie die Bereitschaft, wissenschaftlich abgesicherte Standards der Pflege zu implementieren und sicherzustellen,

» eine weitere Differenzierung der Pflege in Fachbereiche durch den Erwerb von Zusatzqualifikationen mit dem Ziel, in Zukunft Behandlungsaufgaben in größerer Eigenverantwortung übernehmen zu können. ${ }^{143}$

Eine solcherart veränderte Bewertung der Pflege im Krankenhaus und die damit verbundene gesellschaftliche Aufwertung des Pflegeberufes ist notwendig, um eine qualitativ

142 Vgl. Stemmer 2011.

143 Referenzbeispiele hierfür gibt es sowohl in Großbritannien als auch in den skandinavischen Ländern, in denen speziell ausgebildete Pflegekräfte bestimmte medizinische Handlungen in eigener Verantwortung übernehmen. 
hochwertige Pflegepraxis für die Patienten auch in Zukunft durch die Attraktivität des Berufes sicherzustellen. ${ }^{144}$

\subsubsection{Bereich der sozialen und therapeutischen Dienste}

Neben Ärzten und Pflegenden arbeiten im Krankenhaus so unterschiedliche Berufsgruppen wie Hebammen, Physiotherapeuten, Ergotherapeuten, Kunst- und Gestaltungstherapeuten, Psychotherapeuten, Heilpädagogen, Logopäden, Sozialarbeiter und Seelsorger, die sich nicht nur hinsichtlich ihrer Position im klinischen Kontext und in ihrer Arbeitsweise unterscheiden, sondern auch bezüglich der Zielrichtung ihrer Arbeit. Bei aller Differenz gibt es doch ein verbindendes Element: Die Arbeit all dieser Personen ist von großer Bedeutung für ein Krankenhaus, das anstrebt, die verschiedenen körperlichen, psychischen, sozialen und spirituellen Bedürfnisse des Patienten soweit als möglich zu berücksichtigen, da nur das Zusammenwirken aller Berufe im Krankenhaus zu einer ganzheitlichen Wahrnehmung des Patienten als Individuum führt.

144 Das Pilotprojekt „Ausbildung von Arbeitskräften aus Vietnam zu Pflegefachkräften“ im Auftrag des Bundesministeriums für Wirtschaft und Technologie (jetzt: Bundesministerium für Wirtschaft und Energie) zeigt einen Weg auf, dem Fachkräftemangel im Bereich der Pflege im Krankenhaus entgegenzuwirken. Im Herbst 2013 begannen im Rahmen des Projekts erstmals etwa 100 Vietnamesen, die ein Auswahlverfahren und einen Sprachkurs im Heimatland erfolgreich absolviert hatten, mit einer Altenpflegeausbildung in Deutschland. Die Qualifikation nach deutschen Ausbildungsstandards kann auf zwei Jahre reduziert werden, wenn bereits entsprechende Ausbildungsvoraussetzungen aus Gesundheitsberufen vorliegen. Ein umfangreiches Angebot an Begleitung - etwa durch Mentoren und interkulturelles Training - soll die Integration befördern. Da der Gewinnung von Fachkräften innerhalb Europas Grenzen gesetzt sind, kann die Kooperation mit Nicht-EU-Staaten mit Bevölkerungsreichtum und hoher Arbeitsmigration auch im Hinblick auf den Fachkräftemangel in der Krankenhauspflege angezeigt sein. Dabei ist Wert darauf zu legen, dass in den entsendenden Ländern kein Fachkräftemangel entsteht. Ein Überangebot an jungem Fachpersonal in Ländern wie Vietnam bannt diese Gefahr. Die Übertragung des skizzierten Konzepts auf die Krankenpflege ist bereits in Vorbereitung. 
In den genannten Berufsgruppen gibt es wie bei Ärzten und Pflegenden eine unterschiedlich ausgeprägte Beschäftigung mit den ethischen Grundlagen ihrer Arbeit. Je nach Professionalisierungsgrad der Berufe finden sich eigenständige Ethikkodizes, zumindest aber ethische Grundsätze, die teils in der jeweiligen Berufsordnung, teils im Berufsleitbild ausgeführt sind. ${ }^{45}$ Auch theoretische Reflexionen auf das eigene Ethos im Sinne einer Berufsethik gibt es in einigen Berufen aus dem Bereich der Therapie und der sozialen Arbeit. In vielen Professionen gibt es internationale und nationale Übereinkommen zu ethischen Grundsätzen (zum Beispiel soziale Arbeit, Hebammen, Ergotherapie). In den meisten dieser Disziplinen finden (zumindest in Deutschland) allerdings erst in jüngerer Zeit Ethikdiskurse statt, was mit der teils ebenfalls noch jungen Eigenständigkeitsdiskussion der Professionen zu tun hat. Neben dem Rekurs auf medizinethische und pflegeethische Diskurse gibt es eine (Selbst-)Vergewisserung über je berufsspezifische Werte im therapeutischen Alltag.

Wie auch in der Ethik der Pflege (siehe 4.2.2) kann in der sozialen Arbeit an eine Ethik angeknüpft werden, die von einer Care-Praxis als praktizierter Grundüberzeugung ausgeht. Dabei stehen ermutigendes Handeln und achtsame Zuwendung

145 Zum Beispiel: Ethische Richtlinien der Deutschen Gesellschaft für Psychologie und des Berufsverbandes Deutscher Psychologinnen und Psychologen (2005); Ethik-Leitlinien der Deutschen Gesellschaft für Psychoanalyse, Psychotherapie, Psychosomatik und Tiefenpsychologie (2013); Berufsordnung der LogopädInnen vom Deutschen Bundesverband für Logopädie (1998); Leitbild des Deutschen Hebammenverbandes 2011; Internationaler Ethik-Kodex für Hebammen vom Internationalen Hebammenverband (2014); Ethikkodex und Standards zur beruflichen Praxis der Ergotherapie vom Deutschen Verband der Ergotherapeuten (2005); Ethische Grundsätze für Kunst- und GestaltungstherapeutInnen im Deutschen Fachverband für Kunst- und Gestaltungstherapie (2000); Ethik-Kodex der Deutschen Gesellschaft für Erziehungswissenschaft (2010), auf den sich auch die Heilpädagogen beziehen; Berufsethik des Deutschen Berufsverbandes für Soziale Arbeit (2014), auf die sich auch die Deutsche Vereinigung für Soziale Arbeit im Gesundheitswesen bezieht; Ethikkodex professioneller Seelsorger von Rosenberger et al. 2009, über den auch in der Klinikseelsorge diskutiert wird. Siehe auch das internationale und interreligiöse Projekt „Medizinethik in der Klinikseelsorge“ an der Goethe-Universität Frankfurt am Main. 
(care) nicht neben anderen Handlungsabläufen, sondern sie haben in den Prozessen selbst ihren Platz. Ein entscheidender Gesichtspunkt liegt dabei darin, dass das Ziel einer zunehmenden Eigenständigkeit im Handeln, also der Ermöglichung von Selbstbestimmung, gerade dann erreicht wird, wenn die differenzierende Förderung und das gemeinsame Handeln durch achtsame Zuwendung und ermutigendes Handeln bestimmt sind. ${ }^{146}$ Vor diesem Hintergrund haben die therapeutischen und sozialen Berufe im Krankenhaus eine besondere Bedeutung im Hinblick auf die selbstbestimmungsermöglichende Sorge.

Die für die Pflege feststellbaren Konfliktfelder beziehen sich vor diesem Hintergrund in jeweils spezifischer Weise auch auf die meisten der anderen im Krankenhaus tätigen nicht ärztlichen Gesundheitsberufe. Häufig wird angeführt, dass durch die Verkürzung der Verweildauer von Patienten im Krankenhaus bestimmte Leistungen, wie etwa Physiotherapie oder Logopädie, deren Ergebnisse stark mit einer Kontinuität der Behandlungskonzepte und der behandelnden Person korrelieren, kaum sinnvoll angewendet werden können. Dieses Problem bezieht sich zunächst darauf, dass infolge einer durch die möglichst kurze Verweildauer der Patienten bedingten intensiven ärztlichen Diagnostik und Therapie die für die Behandlung durch die nicht ärztlichen Gesundheitsberufe erforderlichen Zeiteinheiten beim Patienten kaum mehr zur Verfügung stehen. Zudem steht aufgrund des erhöhten Patientendurchsatzes kaum genügend Zeit für eine individualspezifisch patientengerechte Behandlung zur Verfügung. Da diese Berufe zudem stark auf eine intensive Kommunikation mit dem Patienten angewiesen sind, indem sie mit dem Patienten Methoden der Selbstbehandlung einüben müssen, spielt ausreichende Zeit eine große Rolle. Schließlich betrifft dieses Problem den Wechsel der Behandlung zum ambulanten

146 Vgl. Conradi 2013, 12. 
Sektor, der oftmals nicht funktioniert oder mit zeitlichen oder konzeptuellen Brüchen verbunden ist.

Eine besondere Problematik betrifft die Sozialdienste und die Seelsorge. Die Sozialdienste klagen darüber, dass in der zur Verfügung stehenden Zeit in vielen Fällen ein tragfähiges soziales Netz für bestimmte Patienten nicht geknüpft werden kann, sodass Patienten häufig in eine kaum zu bewältigende Situation der Selbstversorgung aus dem Krankenhaus entlassen werden. Die Seelsorge ist auf eine intensive Kommunikation mit dem Patienten angewiesen, die unter den gegenwärtigen Umständen kaum zu gewährleisten ist. Auch wenn sich ihre Ergebnisse einer Parametrisierung und Operationalisierung weitgehend verschließen, kann sie doch einen wesentlichen Beitrag für eine selbstbestimmungsermöglichende Sorge um den Patienten und eine angemessene Behandlung im Sinne einer subjektiven Patientenzufriedenheit leisten.

\subsubsection{Bereich der Krankenhausleitung}

Das Handeln der betriebswirtschaftlich und gesundheitsökonomisch ausgebildeten Funktionsträger in der Krankenhausleitung bzw. -administration ist für eine qualitativ hochwertige und selbstbestimmungsermöglichende Krankenhausversorgung von entscheidender Bedeutung. Wenngleich Ehrenkodizes für Manager existieren - etwa Eide, die von Absolventen an Business Schools abgelegt werden und sie auf ethisches Handeln verpflichten ${ }^{147}$-, gibt es für diese Berufsgruppen bislang kein etabliertes, mit der ärztlichen und pflegerischen Profession vergleichbares ethisches Selbstverständnis, das als Kodex für das Handeln im Gesundheitsbereich anwendbar wäre.

147 Vgl. Cabrera 2003. 
Stattdessen kann generell auf Forderungen an die Leitung ${ }^{148}$ in sozialen Organisationen rekurriert werden: Obgleich Krankenhäuser unterschiedlicher Trägerschaft (öffentlich, freigemeinnützig, privat) zuzuordnen und mitunter auf Gewinnerzielung ausgerichtet sind, verweisen der große Anteil der solidargemeinschaftlichen Finanzierung und nicht zuletzt der konstituierende Zweck der Krankenhäuser auf deren Charakter als soziale Organisation.

Leitung ist als organisationsbezogene Aufgabe zu verstehen, die vornehmlich darin besteht, die wechselseitige Beziehung von strukturellen Bedingungen einerseits und individuellem sowie kollektivem Handeln andererseits zu gewährleisten und $\mathrm{zu}$ gestalten. Im Bewusstsein um diese Reziprozität werden Ziele und Aufgaben der Organisation entwickelt. Dazu gehört auch der Umgang mit Fehlern. Insbesondere für soziale und solidargemeinschaftlich finanzierte Organisationen liegt die Forderung nahe, dass Führungspersönlichkeiten wirtschaftliches Handeln mit gesellschaftlichen Zielsetzungen und Ansprüchen abgleichen. Ein ökonomischer Reduktionismus bei der Führung eines Krankenhauses verbietet sich dann für die genannten Professionen.

Management und Verwaltung des Krankenhauses stehen in dem Konflikt, die im Krankenhaus patientengerecht erbrachten Behandlungsleistungen und die hierdurch erzielten Erträge in einem ökonomisch tragfähigen Verhältnis halten zu müssen. Dabei stellt auch die Sicherstellung der ökonomischen Grundlagen des Krankenhauses eine ethische Verpflichtung dar, insofern das Krankenhaus als Versorgungseinrichtung für alle Menschen in einem bestimmten geografischen Einzugsbereich und nicht zuletzt auch als Arbeitgeber für zahlreiche Personen dient. Aber auch die Sicherstellung der ethischen Grundlagen der Behandlung von Patienten ist letztlich für

148 Die Fragen der „Leitung“ oder auch „Führung“ werden im internationalen Schriftgut unter dem Begriff Leadership abgehandelt, der teilweise auch schon in der deutschen Diskussion eingeführt ist, hier aber trotz des damit verbundenen etwas weiteren Blickwinkels nicht zur Anwendung kommt. 
die Existenzsicherung eines Krankenhauses von entscheidender Bedeutung. Management und Verwaltung dürfen bereits aufgrund des Arzt-Patient-Verhältnisses, in dem sie keine Teilnehmer sind, nicht direkt in ärztliche Entscheidungen eingreifen. Gleichwohl bestehen indirekte, weniger offensichtliche Möglichkeiten des Einflusses auf die Behandlung von Patienten, etwa durch Kürzungen des Finanzbudgets von Krankenhausabteilungen oder über die in den meisten Fällen bestehende Dienstaufsicht des Krankenhausmanagements über Ärzte, Pflegende und Mitarbeiter anderer Berufsgruppen, indem bei einem Behandlungsverhalten, das nicht mit den ökonomischen Zielen des Krankenhausmanagements konform geht, Vertragskündigungen ins Spiel gebracht oder vorgenommen werden können. Solche Einflussnahmen stehen allerdings dem Gedanken einer Dienstgemeinschaft entgegen, in die jede Berufsgruppe ihre Kompetenz einbringt, gegenseitig auf die jeweilige Kompetenz der anderen Berufsgruppen vertraut und die im Gesamten das Kompetenzniveau des Krankenhauses auch in der Außendarstellung verkörpert. Es ist daher offensichtlich, dass im Interesse einer angemessenen Behandlung von Patienten eine strukturierte, ständige, transparente und faire Kommunikation zwischen dem Management und der Verwaltung sowie den Angehörigen der im Krankenhaus tätigen Berufsgruppen erforderlich ist. Dabei geht es vor allem auch darum, die ethischen Grundlagen der Patientenbehandlung und die ethischen Verpflichtungen der verschiedenen Berufsgruppen zu reflektieren und angemessen zu berücksichtigen. Neben der Notwendigkeit einer besonderen kommunikativen Kompetenz muss das Management eines Krankenhauses hierfür auch über hinreichende Kenntnisse in den Gesundheitsberufen und die hier etablierten Prozesse einschließlich ihrer ethischen Implikationen verfügen. Dieses besondere Qualifikationsprofil lässt sich vor allem durch die Absolvierung einer Zusatzausbildung, etwa in Ökonomie und Medizin oder Pflege, und eine hinreichende Berufspraxis in 
beiden Fächern sicherstellen, die zum Qualifikationsstandard gehören sollte.

\subsection{Krankenhaus als Organisation}

Neben berufsgruppenspezifischen Ethiken, die sich auf die Verantwortung der Professionen - etwa Ärzte, Pflegekräfte oder Funktionsträger in der Krankenhausleitung bzw. -administration - gegenüber dem Patienten beziehen, ist im komplexen System eines Krankenhauses mit einer Vielzahl interagierender Akteure eine Organisation erforderlich, die auf eine auch ethisch reflektierte rahmengebende Ordnung und das Patientenwohl abstellt. Damit verbunden ist die Annahme, individuell internalisierte Werte durch Anreize für (moralisch) gewünschtes Handeln und Kooperation zwischen einzelnen Akteuren stabilisieren und gegebenenfalls ergänzen zu können. Mögliche Konzepte beziehen sich etwa auf die Anerkennung und Berücksichtigung von Interessen unterschiedlicher Stakeholder oder die Übernahme sozialer bzw. zivilgesellschaftlicher Verantwortung. Kodizes, Leitbilder, Kommissionen oder Schulungen können der Implementierung dienen. Viele der die Berufsgruppen betreffenden Konfliktfelder korrespondieren mit organisationsethischen Strukturen im Krankenhaus. Diese betreffen insbesondere die Möglichkeit transparenter und fachkompetenter Entscheidungen auf der Ebene der Krankenhausleitung sowie die Strukturen einer ethisch angemessenen Entscheidungsfindung.

\subsubsection{Leitungsstrukturen}

Die Kompetenz eines Krankenhauses erweist sich nicht nur in einer positiven ökonomischen Bilanz. Als organisationsethisches Defizit im Hinblick auf die Behandlung von Patienten im Krankenhaus wird immer wieder auf die fehlende Etablierung 
transparenter und partizipativer Entscheidungsstrukturen ${ }^{149}$ hingewiesen. Die Forderung nach einer am Patientenwohl orientierten Behandlung erfordert es, dass die Leitung eines Krankenhauses die Fachkompetenzen aller im Krankenhaus tätigen Berufsgruppen integriert. Hierfür sind Strukturen notwendig, die eine sanktionsfreie professionelle Kommunikation zwischen dem Management und den im Krankenhaus tätigen Berufsgruppen auf gleicher Augenhöhe ermöglichen und zudem geeignet sind, die Kriterien, anhand deren Entscheidungen getroffen werden, transparent zu machen. Letzteres ist auch für eine authentische Außendarstellung des Krankenhauses für Patienten unabdingbar. Solche partizipativen Leitungsstrukturen können insbesondere auch sicherstellen, dass Kernbereiche des diagnostischen, therapeutischen und pflegerischen Handelns nicht primär durch ökonomische Erwägungen bestimmt werden.

Dies betrifft sowohl die Ebene der direkten und individuellen Patientenbehandlung als auch die Gestaltung der Abläufe auf den Stationen und darüber hinaus auch die mittel- und langfristigen Planungsentscheidungen des Krankenhauses. Insbesondere das Verhältnis zwischen der Ertragslage eines Krankenhauses und der Möglichkeit, dass die kaufmännische Leitung auf die ärztliche Behandlung und pflegerische Betreuung der Patienten Einfluss nimmt, muss durchsichtig sein. Eine fachinklusive Leitungsstruktur im Krankenhaus, die kaufmännische Leitung, ärztliche Direktion und Pflegeleitung gleichberechtigt und unbelastet durch arbeitsrechtliche Sanktionsmöglichkeiten in der Krankenhausleitung zusammenführt, kann ein geeignetes Modell darstellen, um eine fachübergreifende Perspektive auf den Patienten auch strukturell abzubilden. Für die Sicherung der Transparenz ist auch die Einrichtung von Gremien im Krankenhaus denkbar, die als Beratungs- und Kommunikationsstellen fungieren und gegebenenfalls zwischen der Leitung und den Mitarbeitern vermitteln.

149 Ausführlicher: Krobath/Heller 2010. 
Die Selbstbestimmung des Patienten bezieht sich insbesondere auch auf den Umgang mit seinen personenbezogenen und gesundheitsbezogenen Daten. Mit der Einführung von elektronischen Krankenhausinformationssystemen, in denen sämtliche Behandlungsdaten gespeichert und einsehbar sind, stellen sich neue Herausforderungen bezüglich eines angemessenen Schutzes dieser Daten vor einer Einsicht durch Unbefugte, aber auch im Hinblick auf den Umfang der Daten, die Behandler und Krankenhausmitarbeiter jeweils einsehen können. Diesbezüglich müssen klare Regeln und technische Voraussetzungen geschaffen werden, die einerseits einen angemessenen Datenschutz sicherstellen, andererseits aber auch effiziente Behandlungsprozesse abteilungs- und sektorübergreifend gewährleisten. Dabei kann zwischen dem berechtigten Interesse des Patienten am Schutz seiner persönlichen Daten und dem gerade mit komplexen Behandlungen verbundenen notwendigen schnellen Austausch der Behandlungsdaten von einzelnen Behandelnden ein Konflikt bestehen.

\subsubsection{Klinische Ethikkomitees}

Die Vorstellungen von Patienten und ihren Angehörigen von einer patientengerechten Behandlung im Krankenhaus sind mit der Erwartung verknüpft, dass das Krankenhauspersonal kompetent ist, in schwierigen Behandlungssituationen $\mathrm{zu}$ ethisch reflektierten und angemessenen Entscheidungen zu gelangen. Diese Erwartung bezieht sich zunächst auf alle in die Patientenbehandlung eingebundenen Mitarbeiter des Krankenhauses, insbesondere aber auch auf ein gut funktionierendes Klinisches Ethikkomitee. Die Zusammensetzung und die Aufgaben von Klinischen Ethikkomitees sind bis auf das 
Bundesland Hessen ${ }^{150}$ nicht gesetzlich festgelegt. ${ }^{151}$ In der Regel haben sie unter anderem die Aufgabe, ethische Fallbesprechungen für Beratungen in individuellen Behandlungsfällen $\mathrm{zu}$ organisieren und durchzuführen, überdies aber auch, Beratung für ethisch relevante Fragen anzubieten, die im Krankenhaus auf verschiedenen Ebenen auftreten können. Hierzu können etwa die Entwicklung von ethischen Leitlinien für das Krankenhaus, Ad-hoc-Beratungen und die Organisation von Fortbildungen im Bereich der klinischen Ethik für die Mitarbeiter der Einrichtung zählen, in selteneren Fällen aber auch eine Beteiligung an der Festlegung der strategischen Ausrichtung des Krankenhauses.

Klinische Ethikkomitees sind allerdings längst nicht in allen Krankenhäusern in Deutschland eingerichtet. Nicht selten werden sie erst zum Zwecke der Erlangung bestimmter Zertifizierungen, die unter anderem eben ein Klinisches Ethikkomitee zur Voraussetzung machen, etabliert. Häufig werden Klinische Ethikkomitees lediglich als additive Elemente aufgefasst, die in die ärztliche und pflegerische Praxis nicht strukturell integriert sind. Hierfür können Desinteresse und Unkenntnis ursächlich sein, häufiger wird allerdings vorgebracht, dass eine strukturierte Beschäftigung mit ethischen Fragen und die hierfür wünschenswerte Ausbildung sowie die Mitgliedschaft in einem Klinischen Ethikkomitee unter den gegenwärtigen Rahmenbedingungen im Krankenhaus eine untragbare zeitliche Belastung darstellten. Zudem erfordert die Etablierung und Integrierung eines Klinischen Ethikkomitees in die Patientenbehandlung den Einsatz zeitlicher und finanzieller Ressourcen, deren Ertrag sich nur schwer in ökonomischen Parametern darstellen lässt. Hinzu kann ein gewisses Misstrauen der Leitungsebenen gegenüber einer strukturierten Ethikarbeit im

$150 § 6$ Abs. 6 des Zweiten Gesetzes zur Weiterentwicklung des Krankenhauswesens in Hessen vom 21. Dezember 2010 (GVBI. I, 587).

151 Siehe zu Aufgaben und Qualität Vorstand der Akademie für Ethik in der Medizin 2010; Zentrale Ethikkommission bei der Bundesärztekammer 2006. 
Krankenhaus kommen, da mit dem Klinischen Ethikkomitee fachliche Kontrollen befürchtet oder ein zusätzliches, klandestines Entscheidungsorgan vermutet wird, das als Störfaktor wirken könne.

Als Folge solcher Befürchtungen werden in Klinischen Ethikkomitees nicht selten bewusst Vertreter der Leitungsebene des Krankenhauses platziert, was sich auf die Unabhängigkeit der Entscheidungen des Klinischen Ethikkomitees in der Regel nicht förderlich auswirkt. Klinische Ethikkomitees wären im Prinzip geeignet, im Krankenhaus ethisch relevante Probleme aufzudecken, Lösungsvorschläge zu erarbeiten und vor allem eine kommunikative Schnittstelle zwischen der Klinikleitung und der Ebene der Mitarbeiter herzustellen. Gleichwohl klagen Mitglieder von Klinischen Ethikkomitees in deutschen Krankenhäusern gegenwärtig oftmals über geringe Beachtung und Wertschätzung in den Einrichtungen. Dem stehen allerdings auch Beispiele für eine sehr gelungene Arbeit gegenüber, wodurch die Sinnhaftigkeit dieses Instruments jedenfalls im Prinzip bestätigt wird. Gerade für eine Einbindung in strategische Beratungen über die Entwicklung des Krankenhauses und im Interesse einer Transparenz von Leitungsentscheidungen für die Mitarbeitenden kann ein unabhängiges Beratungsgremium von Vorteil sein, das dem Klinikmanagement zur Seite steht und dessen Aufgabe darin besteht, Entscheidungen im Hinblick auf die Patientenversorgung zu prüfen, Anregungen aus der Einrichtung aufzunehmen und gegenüber der Klinikleitung vorzubringen und bei Kontroversen $\mathrm{zu}$ beraten. $\mathrm{Ob}$ diese Funktion von einem Klinischen Ethikkomitee wahrgenommen werden kann bzw. sollte oder von einem anderen Gremium, hängt von den Bedingungen im jeweiligen Krankenhaus ab. 


\subsection{Fort- und Weiterbildung des Krankenhauspersonals}

Eine qualitativ und quantitativ angemessene Fort- und Weiterbildung des Krankenhauspersonals ist eine unabdingbare Voraussetzung für das Angebot einer den medizinischen Standards entsprechenden Behandlung. Die knappe Finanzsituation mancher Einrichtungen und die hierdurch bedingten dünnen Personaldecken können Gründe dafür darstellen, dass eigene Angebote der Fort- und Weiterbildung des medizinischen Personals seitens der Einrichtung entweder unterbleiben oder verringert werden oder dass Mitarbeitern eine Teilnahme an entsprechenden auswärtigen Veranstaltungen untersagt werden muss oder diese selbst auf eine Teilnahme verzichten, um die Patientenversorgung in ihrer Einrichtung nicht durch ihre Abwesenheit zu gefährden. Zwar verlangen die Landesärztekammern von jedem im Krankenhaus tätigen Arzt den jährlichen Nachweis eines festgelegten Kontingents an Fortbildungsstunden, jedoch ist die Wahl solcher Fortbildungsveranstaltungen thematisch nicht festgelegt. Im Pflegebereich existieren solche Auflagen nicht; die Fortbildung findet auf freiwilliger Basis statt. Im Interesse der Sicherstellung einer guten Behandlung sollten Einrichtungen bzw. Träger mit einem bestimmten Spektrum an Behandlungsangeboten daher gewährleisten, dass ein diesem Spektrum entsprechendes Fortbildungsangebot zur Verfügung steht, und dafür Sorge tragen, dass die Mitarbeiter diese Angebote wahrnehmen können.

\subsection{Patientengruppen mit besonderen Bedarfen}

\subsubsection{Kinder und Jugendliche}

Nach den Angaben der Gesellschaft der Kinderkrankenhäuser und Kinderabteilungen in Deutschland und der Deutschen 
Gesellschaft für Kinder- und Jugendmedizin wurde seit 1991 fast jede fünfte Kinderabteilung geschlossen, vier von zehn Betten in der stationären Kinder- und Jugendmedizin wurden gestrichen. ${ }^{152} 1991$ gab es 440 Abteilungen für Kinderheilkunde mit 31.708 Betten, 2013 waren es noch 364 Abteilungen mit 19.199 Betten. Die Anzahl der Abteilungen für Kinderchirurgie schrumpfte von 99 auf 80. Als Konsequenz dieser Entwicklung nimmt die flächendeckende Versorgung ab. Nach den Empfehlungen von Verbänden der Kinder- und Jugendmedizin und der Deutschen Akademie für Kinder- und Jugendmedizin sollte eine Kinderklinik oder eine Abteilung für Kinder-und Jugendmedizin in maximal 40 Minuten Fahrzeit und 30 Kilometer Fahrstrecke für den Patienten erreichbar sein. Demnach sollten Abteilungen nicht mehr als 80 Minuten Fahrzeit bzw. 60 Kilometer voneinander entfernt sein. Diese Kenngrößen werden bereits teilweise nicht mehr erreicht. Trotz der demografischen Entwicklung ging allerdings die Anzahl von Kindern, die eine stationäre Versorgung benötigen, nicht zurück, sondern blieb relativ konstant.

Im Vergleich mit der Erwachsenenmedizin müssen Abteilungen für Kinder- und Jugendmedizin ein weitaus höher differenziertes Leistungsspektrum bedienen. Während in Abteilungen der Erwachsenenmedizin im Durchschnitt ca. 200 verschiedene Fallpauschalen angewandt werden, umfasst das Leistungsspektrum einer Kinderklinik 400 bis 500 DRGs. Ein Teil dieser DRGs ist nicht kinderspezifisch, sondern kommt aus der Erwachsenenmedizin. Generell fällt bei Kindern jüngeren Alters ein deutlich höherer Pflegeaufwand an, ferner längere Gesprächszeiten mit den Angehörigen, ein höherer Zeitaufwand bei Untersuchungen (zum Beispiel beim Röntgen) sowie eine psychosoziale Betreuung insbesondere auch bei chronischen Krankheiten. Die Personalkosten

152 Zu den Zahlen in diesem und im folgenden Absatz siehe Presseinformation der Deutschen Gesellschaft für Kinder- und Jugendmedizin vom 11. April 2014: http://www.dgkj.de/service/meldungsarchiv/meldungen/2014/ presseinfo_rettet_die_kinderstation [28.09.2015]. 
in Kinderkliniken betragen ca. 80 Prozent der Gesamtkosten der Behandlung und liegen damit ca. 30 Prozent höher als in der vergleichbaren Erwachsenenmedizin. Da seltene Erkrankungen häufig in den ersten Lebensjahren festgestellt werden, werden in der Kinder- und Jugendmedizin viele solcher Fälle betreut. Kinder mit seltenen Erkrankungen benötigen allerdings häufig eine aufwendige Versorgung; zudem gibt es in diesen Fällen aufgrund der geringen Fallzahl selten eine eigene angemessen kalkulierte Fallpauschale. Zudem findet die Behandlung von Kindern wenn irgend möglich in ambulanter Betreuung durch das Krankenhaus statt, wobei die Vergütung ambulanter Eingriffe deutlich unter dem stationären Vergütungssatz liegt. Überdies wird ein stationärer Aufenthalt bei Kindern nicht zuletzt aus psychologischen Gründen auf die geringstmögliche Zeit beschränkt, wodurch in etwa 25-30 Prozent der stationären Behandlungen die untere Grenzverweildauer unterschritten wird, was mit hohen Abschlägen für die Erlöse des Krankenhauses verbunden ist.

Ein weiteres Problem zeigt sich, so die Deutsche Gesellschaft für Kinder- und Jugendmedizin und die Gesellschaft der Kinderkrankenhäuser und Kinderabteilungen in Deutschland, bei den hohen Fixkosten in den Kinderkliniken, die im DRG-Vergütungssystem nicht berücksichtigt sind. Während in der Erwachsenenmedizin für die ständige Verfügbarkeit der stationären Versorgungsleistung ca. 25 Prozent des Budgets angesetzt werden, liegt bei Kinderkliniken der Anteil für diese Vorhaltekosten bei bis zu 40 Prozent des Budgets, da der Anteil an planbaren Leistungen in der stationären Pädiatrie nur ca. 20 Prozent ausmacht und die Notfallquote durch akut auftretende Erkrankungen mit 50 Prozent sehr hoch ist. In der Folge ist es in Krankenhäusern häufig notwendig, die stationäre Behandlung von Kindern und Jugendlichen aus der Erwachsenenmedizin querzusubventionieren.

Aufgrund des akuten Risikos, dass Kinderklinken und -abteilungen aus finanziellen Gründen geschlossen werden, haben die Bundesärztekammer, die Deutsche Gesellschaft für 
Kinder- und Jugendmedizin und die Gesellschaft der Kinderkrankenhäuser und Kinderabteilungen in Deutschland 2014 die Informationskampagne „Rettet die Kinderstation!“ gestartet, die auf die bundesweit bedrohte Krankenhausversorgung von Kindern und Jugendlichen hinweist und Lösungsmöglichkeiten aufzeigt.

Der Vergleich mit der Erwachsenenmedizin und das offensichtliche Durchschlagen des Ziels von Einsparungen im Gesundheitssystems auf den personal- und kostenintensiven Bereich der Kinderheilkunde werfen die Frage auf, inwieweit es gerechtfertigt ist, Kinder in einem marktorientierten Finanzierungssystem durch Fallpauschalen in Konkurrenz zu Erwachsenen um begrenzte Ressourcen zu stellen. Gerechtigkeit im Gesundheitswesen wird vor allem im Sinne eines diskriminierungsfreien gleichen Zugangs zum Gesundheitssystem und einer Gleichheit der medizinischen Versorgung im Sinne einer gerechten Ressourcenverteilung angesehen. Wie jeder Bürger haben auch Kinder ein Recht auf eine qualifizierte medizinische Versorgung. Betrachtet man die Folgen der Schließung von Kinderabteilungen und Kinderkrankenhäusern in Form von längeren Anfahrten und der Wohnortferne, längeren Wartezeiten, fremden Ärzten und Pflegenden, ist festzustellen, dass diese Faktoren zwar nicht das Recht der Kinder auf medizinische Versorgung verändern, wohl jedoch die Bedingungen, unter denen Kinder dieses Recht wahrnehmen können. ${ }^{153}$ Während diese Faktoren bei Erwachsenen - jedenfalls in der Regel und bis zu einem gewissen Ausmaß - nicht als unzumutbar empfunden werden, bestehen bei Kindern diesbezüglich Zweifel, die in ihrem Entwicklungsstadium gründen: Kinder sind nicht selbstbestimmt wie Erwachsene, können ihre Rechte nicht selbst wahrnehmen und haben andere Bedürfnisse, da sie sich nicht in der gleichen Weise wie Erwachsene zu den genannten Bedingungen verhalten können. Kinder können daher zu Recht von staatlichen Institutionen eine besondere Art

$153 \mathrm{Vgl}$. Wiesemann/Lenk 2006, 49. 
der Fürsorge erwarten, die es auch rechtfertigen kann, Kinder einer unmittelbaren Konkurrenz mit Erwachsenen um knappe Güter im Gesundheitswesen zu entheben. ${ }^{154}$

Eine Lösung der skizzierten Probleme könnte darin bestehen, dass die die Kinder- und Jugendmedizin charakterisierenden Faktoren, wie zum Beispiel sehr kurze Verweildauern von Kindern im Krankenhaus oder eine vergleichsweise sehr aufwendige pflegerische und psychologische Betreuung, angemessen, das heißt kostendeckend im DRG-System berücksichtigt werden. Damit wäre zumindest die unmittelbare Auswirkung der Konkurrenzsituation entschärft, wenngleich diese im Prinzip nicht aufgehoben wäre. Letzteres könnte erreicht werden, wenn die Kinder- und Jugendmedizin komplett von dem Fallgruppen-Vergütungssystem der Erwachsenenmedizin entkoppelt würde und entweder ein kinderspezifisches eigenes DRG-System etabliert oder für die stationäre Kinderund Jugendmedizin ganz andere Abrechnungsmodi, etwa tagesgleiche Pflegesätze, eingeführt würden. Die fortdauernde Schließung von Fachabteilungen für Kinder- und Jugendmedizin in Krankenhäusern aus finanziellen Gründen stellt unter dem Aspekt der Gerechtigkeit jedenfalls eine höchst problematische Entwicklung dar.

\subsubsection{Patienten in hohem Lebensalter}

In vielen Abteilungen der Krankenhäuser befinden sich zunehmend mehr ältere und hochbetagte Patienten. Auf die Frage, wer als alt gilt, werden unterschiedliche Antworten gegeben. Die Disziplinen der Biologie, Soziologie und Psychologie wenden diesbezüglich jeweils unterschiedliche Kriterien an. Nach der Definition der Weltgesundheitsorganisation gilt als alt, wer das 65. Lebensjahr vollendet hat. Allerdings ist das kalendarische Alter für die Bestimmung der Zugehörigkeit

154 Vgl. ebd., 53. 
zur Gruppe der alten Menschen offenbar wenig hilfreich. Als genereller Indikator kann gelten, dass alte Menschen für die Dinge des Alltags zunehmend mehr Zeit benötigen. Zunächst wird dieser Mehrbedarf durch erlernte Effizienzstrategien und Erfahrung kompensiert, später dann wird der erhöhte Zeitbedarf manifest. Altersbedingten körperlichen Einschränkungen kommt in der Regel kein objektiver Krankheitswert zu. Im Alter können Erkrankungen auftreten, für die ein hohes Alter nicht ursächlich ist (zum Beispiel die Entwicklung eines Dickdarmtumors). Allerdings erkranken Menschen im Alter häufiger, manche Erkrankungen sind altersassoziiert (zum Beispiel ein Oberschenkelhalsbruch), und sowohl die Krankheitssymptome als auch der Umgang der Betroffenen mit der Erkrankung können sich mit altersbedingten Einschränkungen in komplexer Weise verbinden.

In einer auf ökonomische und zeitliche Effizienz ausgerichteten Krankenhausmedizin können diese Zusammenhänge für alte Menschen, die sich im Krankenhaus behandeln lassen müssen, erhebliche Bedeutung gewinnen. Heilungsprozesse im Alter dauern aus physiologischen Gründen länger. Alte Patienten können oft dem zeitlich eng getakteten Alltag im Krankenhaus kommunikativ kaum entsprechen (zum Beispiel wegen Schwerhörigkeit, längeren Phasen des Nachdenkens, langsamer Artikulation und der Notwendigkeit der Klärung unbekannter Begrifflichkeit beim Anamnesegespräch). Zudem können alte Menschen den Erfordernissen des Krankenhausalltags prozedural oft nicht gerecht werden (zum Beispiel bei dem selbstständigen Aufsuchen von diagnostischen Fachabteilungen im Krankenhaus). Sie bedürfen daher besonderer Hilfe und erhöhter zeitlicher und persönlicher Zuwendung. Aufseiten des Krankenhauses drückt sich dies in einer längeren Verweildauer, einem erhöhten Personalbedarf und in verlängerten Prozessabläufen aus. Die vermehrte Zeit, die alte Menschen benötigen, kann sich aus ökonomischer Perspektive für das Krankenhaus als Nachteil erweisen. Zudem ergeben sich medizinische Abwägungen bei alten Patienten in anderer Weise 
als bei jüngeren, etwa in Hinblick auf eine größere Zurückhaltung bei invasiven Therapien und Prozeduren wegen eines mit zunehmendem Alter allgemein steigenden Risikos solcher Eingriffe, wodurch sich allerdings die Vergütung für das Krankenhaus verringern kann. Überdies muss eine altengerechte medizinische Behandlung einen Paradigmenwechsel von der Defizitorientierung (Beseitigung gesundheitlicher Defizite) zu einer Ressourcenorientierung (Einsatz und Förderung der Fähigkeiten, die bei dem alten Patienten vorhanden sind) vollziehen, was ebenfalls eine verstärkte personelle Zuwendung erfordert. Die Behandlung alter Patienten im Krankenhaus ist vor diesem Hintergrund oftmals nicht gewinnbringend. Diese Umstände können zu einem tendenziell erschwerten Zugang alter Menschen zur Krankenhausmedizin führen.

\subsubsection{Patienten mit geriatrietypischen Erkrankungen}

Der geriatrische Patient ist charakterisiert durch seine Multimorbidität, das Erfordernis einer Polypharmakotherapie, die Chronifizierung der Krankheiten, Immobilitätssyndrome und Funktionseinbußen, die einander oft negativ beeinflussen. Hinzu kommen psychosoziale Faktoren, die meist mit den somatischen Problemen des älteren Patienten verbunden sind. Allen diesen Faktoren muss bei der Krankenhausbehandlung des älteren Menschen Rechnung getragen werden.

Die Geriatrie hat sich in Deutschland erst ab der Mitte der 1980er-Jahre den in anderen Ländern bereits vorhandenen professionellen Standards, die diesbezüglich mindestens zehn Jahre voraus waren, angenähert. Aus einer „perspektivlosen Verwahrmedizin“" 155 entwickelte sich ein ganzheitliches und funktionell ausgerichtetes Behandlungskonzept, das ergänzend zur Akutbehandlung bei geriatrischen Patienten

155 Borchelt 2004, 2. 
frühzeitig auch rehabilitative Methoden einsetzt. Die Akutgeriatrie vereinigt die Gleichzeitigkeit von Akutmedizin und Rehabilitation. Dieser frührehabilitative Ansatz führt allerdings dazu, dass es vom Leistungsgeschehen her schwierig ist, zwischen der Akutbehandlung und der Rehabilitation scharf zu trennen. Dementsprechend besteht die Situation, dass die geriatrisch-frührehabilitative Komplexbehandlung derzeit nicht nur in Krankenhäusern mit Versorgungsvertrag nach $\$ 109$ SGB V auf der Basis von $₫ 39$ SGB V, sondern auch in Rehakliniken mit Versorgungsvertrag nach $\$ 111$ SGB V auf der Basis von $₫ 40$ SGB V durchgeführt und abgerechnet wird. Diese weniger aus medizinischer Perspektive als vor allem aus leistungsrechtlicher Sicht heterogene Situation führt im auch für die Geriatrie geltenden Abrechnungssystem der DRGs in den Versorgungsverträgen in einigen Bundesländern dazu, dass jede Krankheitsphase eines geriatrischen Patienten nach ihrer Akuität unterschieden wird. Tritt etwa nach den Kriterien des Versorgungsvertrags des Landes Niedersachsen nach einer Akutbehandlung eines geriatrischen Patienten Rehabilitationsfähigkeit ein, ist auf „\$111“ umzustellen; wird diese Phase zum Beispiel von einer akuten Lungenentzündung wieder unterbrochen, ist auf „\$109“ zurückzustellen, bis erneut Rehabilitationsfähigkeit erreicht ist. Kritisiert wird, dass damit das reale Leistungsgeschehen am Patienten, der von alledem nichts bemerkt, weil er immer im gleichen Bett bleibt und von demselben Team auf derselben Station behandelt wird, völlig verschleiert wird. Wird hinterher jeweils nur ein Versorgungsbereich ausgewertet und für die Vergütungsverhandlungen herangezogen, ergibt sich sowohl hinsichtlich der Fallzahlen als auch des Leistungsspektrums ein verzerrtes Bild. ${ }^{156}$ Solche systematischen Verzerrungen bei einer als besonders vulnerabel einzustufenden Patientengruppe können vor dem Hintergrund des Systems der Abrechnung nach Fallpauschalen ein gravierendes Gerechtigkeitsproblem begründen.

156 Ebd., $4 \mathrm{ff}$. 
Diesbezüglich wird dringender Bedarf für die Entwicklung angemessener Lösungen gesehen. ${ }^{157}$

Neben diesen Problemen in der Versorgungsstruktur ergeben sich auch Fragen hinsichtlich einer gerechten Berücksichtigung geriatrischer Patienten in den Fallgruppen. Die Zuweisung erfolgt vor allem auf der Basis der Hauptdiagnose und der Komorbidität bzw. des Komplexitätsgrades. Dabei erweisen sich kognitive Einschränkungen im Sinne einer Demenz oder einer beginnenden Demenz, die gerade bei geriatrischen Patienten häufig anzutreffen sind, im Hinblick auf eine kostendeckende Behandlung als besonders problematisch. Beklagt wird, dass der hohe Versorgungsbedarf und die notwendigen erheblichen zusätzlichen Aufwendungen bei den betroffenen Patienten weder bei Demenz als Hauptdiagnose noch als Nebendiagnose in den Fallpauschalen ausreichend, das heißt kostendeckend abgebildet sind. Zudem ist das wünschenswerte Ziel einer integrierten Versorgung geriatrischer Patienten im Krankenhaus bereits aus den oben genannten Gründen altersassoziierter funktioneller Einschränkungen unter den gegebenen Umständen kaum zu erreichen. Überdies stellen sich bei geriatrischen Patienten, insbesondere solchen mit Demenz, die mit einer fragmentierten Versorgung im stationären und ambulanten Bereich verbundenen Probleme in besonderer Schärfe. Nicht zuletzt ist auch zu fragen, wie bei geriatrischen Patienten die letzte Lebensphase und das Sterben im Krankenhaus würdig gestaltet werden können und wie sich dies in einem nach Fallpauschalen gegliederten Abrechnungssystem abbilden lässt.

Zusammenfassend lässt sich feststellen, dass alte Patienten und geriatrische Patienten im Krankenhaus besonderer Aufwendungen bedürfen, die sich unmittelbar in einer intensivierten personalen Zuwendung niederschlagen. Diese Patienten haben wie alle anderen Patienten ein Recht darauf, im

157 Schulz/Kurtal/Steinhagen-Thiessen 2008; Füsgen 1996; §§ 108, 109, 111 SGB V. 
Solidarsystem der Krankenversicherung adäquat behandelt zu werden. Ihre Behandlung kann sich nicht an den für jüngere Patienten geltenden Kriterien orientieren, weil sich bereits durch den häufig nicht linearen Krankheitsverlauf bzw. die Multimorbidität regelhaft ein wechselhaftes und oftmals nicht ganz eindeutiges Bild ergibt und das Vorliegen bzw. das Ende der im Gesetz geforderten akutstationären Behandlungsbedürftigkeit nur schwierig eindeutig zu bestimmen ist. Zudem ergeben sich mit dem häufigen Auftreten demenzieller Veränderungen im Vergleich mit jüngeren Patienten spezifische gesundheitliche Probleme. Es stellt sich die Frage, wie diese Patientengruppen gerecht in einem Abrechnungssystem nach Fallpauschalen, das unterschiedliche Patientengruppen im Prinzip in Konkurrenz zueinander setzt, berücksichtigt werden können.

Eine Lösung könnte darin bestehen, dass Zusatzentgelte für bestimmte komplexe multimorbide Krankheitsbilder bereitgestellt werden und alten Patienten im Krankenhaus eine abrechenbare persönliche pflegerische Betreuung zur Seite gestellt wird, die bei Bedarf aktiviert werden kann. Diese Betreuung müsste dann ebenfalls über spezielle DRGs finanziert werden.

\subsubsection{Patienten mit Demenz}

Für Patienten, die wegen einer somatischen Erkrankung im Krankenhaus behandelt werden müssen und gleichzeitig an einer bereits festgestellten oder erst dann im Krankenhaus diagnostizierten Demenz leiden, ergeben sich oft erhebliche Probleme. Die Deutsche Alzheimer Gesellschaft beklagt, dass die Allgemeinkrankenhäuser kaum auf die angemessene Umgangsweise mit Demenzpatienten vorbereitet sind, und empfiehlt ihren Mitgliedern, bei einer Krankenhausaufnahme vorbereitete Auskunftsbögen über die vorhandenen Fähigkeiten 
und Besonderheiten mitzuführen. ${ }^{158}$ Oftmals werde bei der Aufnahme aber weder der Grad der demenziellen Erkrankung noch das genaue Profil der noch vorhandenen Fähigkeiten, Selbstbestimmungskompetenzen und Besonderheiten erfasst. Entmündigende Pflege, die die noch vorhandenen Selbstständigkeitspotenziale ignoriert, und Behandlungen und Maßnahmen ohne Kommunikation, die die Betroffenen noch mehr als die ohnehin fremde Situation verwirren, seien die Folge. Fähigkeiten, die zu Hause noch bestanden, würden schnell verlernt und könnten nach Krankenhausaufenthalten nur mühsam wiedererlangt werden. Die meisten Schwierigkeiten beim Umgang mit den Patienten entstünden im Verlauf des Krankenhausaufenthaltes dann aber weniger aufgrund der kognitiven Einschränkungen, sondern meist aufgrund bestimmter Verhaltensweisen wie Herumlaufen, Schreien oder permanentes Nachfragen, deren Sinn und deren Ursachen oftmals nicht erkannt würden. Auch fehle das Wissen über die richtigen Strategien, um mit solchen Verhaltensweisen ohne den Einsatz von Psychopharmaka angemessen umzugehen. ${ }^{159}$

Für die Situation von Demenzerkrankten im Krankenhaus werden folgende Probleme benannt, auf die das Krankenhaus reagieren müsste:

» kognitive Beeinträchtigungen, die die Verständigung erschweren und Symptombeschreibungen seitens des Patienten und seine Rückmeldungen zu Wirkungen und Nebenwirkungen von Medikamenten unsicher machen,

» fremde Umgebung und veränderter Tagesablauf sowie nicht vertraute Abläufe und Handlungen, die unter anderem Angst, Abwehr oder Aggression auslösen können,

\footnotetext{
158 Vgl. Deutsche Alzheimer Gesellschaft 2013; Deutsche Alzheimer Gesellschaft 2015.

159 Teschauer 2014; Teschauer 2015.
} 
» fehlende Abwechslung, Beschäftigungs- und Bewegungsmöglichkeit, die unter anderem zu Anspannung und Unruhe führen können,

» fehlende Bezugspersonen, die die Besonderheiten des Patienten kennen. ${ }^{160}$

Hinzu kommt, dass eine knappe und funktionale Kommunikation, die oft mit Forderungen oder auch Zurechtweisungen verbunden ist, zu weiteren Problemen führen kann. Die am häufigsten benannten Verhaltensauffälligkeiten als Reaktion auf die unverstandene Krankenhauswelt bestehen neben den oben genannten Hilferufen, ziellosem Umherlaufen, körperlicher oder verbaler Aggression und Schreiattacken bisweilen auch in Wahnvorstellungen, Apathie oder Enthemmung. In Krankenhäusern, die weder strukturell noch personell auf diese besondere Patientengruppe eingestellt sind, kommt es zu Überforderungen des Personals, unangemessener Behandlung (beispielsweise Fixierung) und letztlich auch zur Ablehnung solcher Patienten.

Diese Probleme erhalten eine besondere Brisanz, wenn man sich die prognostizierten Zuwachsraten im Bereich alter und hochaltriger Patienten und deren Risiken, gleichzeitig an einer Demenz zu erkranken, vergegenwärtigt. So wird nach Berechnungen des Statistischen Bundesamtes der Anteil der über 80-jährigen Krankenhauspatienten 2020 19,5 Prozent und 2030 20,7 Prozent, der Anteil der 60- bis 80-jährigen Krankenhauspatienten 2020 35,6 Prozent und 2030 41,7 Prozent betragen. ${ }^{161}$ Die Prävalenzraten für Demenzerkrankte in Akutkrankenhäusern werden je nach Altersgruppenbezug unterschiedlich angegeben. ${ }^{162}$ Während eine prospektive Untersuchung für Deutschland auf 28 Prozent demenziell erkrankter Krankenhauspatienten über 60 Jahre in Akutkrankenhäusern

160 Teschauer 2015, 15 .

161 Statistische Ämter des Bundes und der Länder 2010, 15.

162 Pinkert/Holle 2012. 
kommt ${ }^{163}$, gibt eine internationale Studie einen Prozentsatz von 18 Prozent aller Krankenhauspatienten über 65 Jahre an ${ }^{164}$.

Für diese große und in Zukunft wachsende Gruppe der Patienten mit Demenz gilt es, geeignete Voraussetzungen zu schaffen, um sie unter Wahrung ihrer Würde und noch möglichen Selbstbestimmung im Krankenhaus adäquat behandeln und versorgen $\mathrm{zu}$ können. ${ }^{165}$

Neben der Einführung einer verbesserten Frühdiagnostik durch entsprechende Screeningverfahren sind im Wesentlichen bessere Aufnahmebedingungen in den Krankenhäusern zu fordern, bei denen von den Angehörigen Besonderheiten des Patienten systematisch erfragt werden und der Stand der vorhandenen Selbstpflegekompetenzen und Selbstbestimmungsmöglichkeiten ermittelt wird. Des Weiteren sollten die Abläufe im Aufnahmeverfahren beschleunigt werden und die Rooming-in-Möglichkeiten für Angehörige erweitert werden. ${ }^{166}$ Zudem sind Schulungen des Personals notwendig, in denen Fachwissen über Demenz vermittelt wird, aber auch Kommunikationsfähigkeiten trainiert werden und problematische Standardsituationen (zum Beispiel: ein dementer Patient irrt im Krankenhaus umher oder reißt sich die Verweilkanülen aus den Venen) anhand von Best-Practice-Beispielen thematisiert werden. ${ }^{167}$ Ziel ist hierbei auch, das Belastungserleben des Pflegepersonals bei der Versorgung demenzkranker Patienten im Krankenhaus zu reduzieren.

163 Trauschke/Werner/Gerlinger 2009, 386.

164 Arolt/Driessen/Dilling 1997, 208.

165 Für den Bereich „demenzsensibles Krankenhaus“ sei auf das Programm „Menschen mit Demenz im Akutkrankenhaus“ der Robert Bosch Stiftung verwiesen (http://www.bosch-stiftung.de/content/language1/html/37166. asp [22.02.2016]), für den Bereich „barrierearmes Krankenhaus“ (wobei es bei Barrieren um architektonische wie kommunikative geht) auf die Stiftung Gesundheit Fördergemeinschaft (http://www.stiftung-gesundheitfoerdergemeinschaft.de [22.02.2016]).

166 Deutsche Alzheimer Gesellschaft 2013, 16.

$167 \mathrm{Vgl}$. hierzu unter anderem das Programm „Demenzkranke Menschen in individuell bedeutsamen Alltagssituationen“ (DEMIAN), das im Institut für Gerontologie der Universität Heidelberg in den Jahren 2004 bis 2010 entwickelt wurde. Vgl. auch Berendonk et al. 2011. 
Die Deutsche Alzheimer Gesellschaft geht davon aus, dass es einer Änderung der Haltung im Gesamtsystem Krankenhaus bedarf, um die Bedarfe der Gruppe der Demenzpatienten besser abzudecken. Als wesentliche Handlungsoptionen für die Weiterentwicklung eines Krankenhauses zu einem „demenzsensiblen Krankenhaus" werden dabei genannt:

\A Anpassung des Milieus und der Abläufe auf einer Station an die Bedürfnisse der Menschen mit Demenz,

» Einbindung von Angehörigen und Helfern,

»S Schulung und Fortbildung des Personals,

» Bereitstellen von Beschäftigungs- und Bewegungsmöglichkeiten. ${ }^{168}$

Es wird betont, dass es neben der Vermittlung von Fachwissen und Handlungssicherheit zum Abbau von Ängsten auch um eine Änderung im Sinne einer positiven Grundhaltung gegenüber der Demenz als Krankheit und dem Demenzbetroffenen geht, die ihn weder zum Kind degradiert, noch als reinen Pflegefall sieht. Diese Änderung setzt bei einzelnen Mitarbeitern Einfühlungsvermögen voraus, aber auch die Bereitschaft, die emotionalen Mitteilungen der Betroffenen stärker wahrzunehmen als ihre kognitiven. ${ }^{169}$ Des Weiteren ist die Gewährleistung einer adäquaten Zeit, die für den einzelnen Patienten aufgebracht werden muss, eine wesentliche Voraussetzung für die Weiterentwicklung eines Krankenhauses zu einem demenzsensiblen Krankenhaus. Ob die Versorgung demenzbetroffener Krankenhauspatienten besser durch Betten, die in den verschiedenen Fachabteilungen integriert sind, erfolgt oder in einer interdisziplinären Station, in der ein demenzfreundliches Milieu durch ein ganzes Bündel an Maßnahmen und zusätzliche Tagesstrukturierungen geschaffen werden kann, ist derzeit nicht abschließend geklärt.

169 Siehe dazu ausführlicher: Deutscher Ethikrat 2012. 


\subsubsection{Patienten mit Behinderung}

Patienten mit Behinderung und ihre Angehörigen klagen häufig darüber, dass sie in Krankenhäusern entweder abgewiesen oder so wenig fachgerecht behandelt werden, dass sie in wohnortfernen Kliniken ausweichen müssen.

Darüber hinaus wird berichtet, dass viele Krankenhäuser unabhängig von den Selbstkompetenzen der Betroffenen die Begleitung durch eine weitere Person bei der Krankenhausaufnahme verlangen ${ }^{170}$ und keine Möglichkeit anbieten, vor der Aufnahme die entsprechende Station kennenzulernen, um notwendige Vorkehrungen für die Zeit des Aufenthaltes zu treffen $^{17}$. Eine systematische und angemessene Einschätzung der Selbstpflegefähigkeiten und spezifischen Unterstützungsbedarfe nach der Aufnahme werde oft unterlassen und führe sowohl zu einer teilweisen Überversorgung und entmündigenden Pflege als auch zu einer Unterversorgung durch Unterlassung notwendiger Hilfe. ${ }^{172}$ In der Stellungnahme der ZEKO zur UN-Behindertenrechtskonvention heißt es: „Menschen mit Behinderung machen die Erfahrung, dass auf den zeitlichen Mehraufwand, den sie verursachen (zum Beispiel wenn sie längere Zeit zum Anund Auskleiden oder besondere Kommunikationshilfen benötigen), ungehalten reagiert wird. “'733 Bemängelt wird des Weiteren, dass die Kommunikation im Krankenhaus auf den kompetenten Patienten zugeschnitten sei. Für Patienten mit Artikulationsproblemen, Patienten, die längere Zeit für Antworten brauchen, oder Patienten, die sich nonverbal verständigen, bestehe aber weder die dafür erforderliche Zeit noch die Expertise. ${ }^{174}$

Für den Umgang mit Menschen mit Behinderung können die Prinzipien und Aussagen der UN-Behindertenrechtskonvention als Grundlage gelten (siehe 3.3.1). Die Konvention

\footnotetext{
170 Lachetta et al. 2011, 143 .

171 Budroni et al. 2006, 127.

172 Forum selbstbestimmter Assistenz behinderter Menschen 2007.

173 Zentrale Ethikkommission bei der Bundesärztekammer 2010, A298.

174 Schmidt 2010.
} 
spricht den Menschen mit Behinderung nicht nur die "gleiche“ Gesundheitsbehandlung wie nicht behinderten Menschen zu, sondern auch Gesundheitsleistungen, die von ihnen speziell wegen ihrer Behinderung benötigt werden (Art. 25 b), und Gesundheitsdienste, die ihrer "Habilitation und Rehabilitation“ dienen (Art. 26), um ein Höchstmaß an Unabhängigkeit sowie volle und gleichberechtigte Teilhabe zu erreichen.

Vor dem Hintergrund des Gebots des gleichen Zugangs und der gerechten Verteilung lassen sich für das Krankenhaus die Konflikte mit drei Fragen umreißen:

\W Wie kann die Selbstbestimmung von Menschen mit Behinderung im Krankenhaus, die aufgrund ihrer kognitiven Einschränkungen einer Assistenz bei der Selbstbestimmung bedürfen, vor dem Hintergrund des wachsenden ökonomischen Drucks gewährleistet werden?

» Wie kann bei Menschen mit Behinderung, bei denen die Behinderung nicht der Grund für die aktuelle Krankenhausbehandlung ist und die unmittelbare Behandlung der mit der Behinderung verbundenen Symptome eine spezialisierte Versorgung nicht erforderlich macht, die medizinische und pflegerische Versorgung im Sinne der Inklusion im Rahmen des regulären Behandlungsangebots der Krankenhäuser sichergestellt werden?

》Wie kann ein angemessener Ausgleich erreicht werden zwischen der flächendeckenden Versorgung von Menschen mit Behinderung in den Allgemeinkrankenhäusern (einer Art Generalisierung der Angebote für die besonderen Bedarfe dieser Patientengruppe) und der Spezialisierung bestimmter Krankenhäuser auf die Behandlung spezifischer Untergruppen, die, wie zum Beispiel im Falle bestimmter genetischer Syndrome, eine hohe Expertise voraussetzen?

Erst langsam setzt ein Prozess der Vergegenwärtigung dieser Fragen ein. Bisher gibt es nur wenige Programme für 
bedarfsgerechte Behandlungen, die meist von Stiftungen, aber auch von einzelnen Bundesländern finanziert sind.

Bei Lösungsansätze muss berücksichtigt werden, dass Menschen mit Behinderung bei einem stationären Krankenhausaufenthalt eine Pflege und Behandlung benötigen, die besondere Anforderungen an Pflegende wie Ärzte stellt. Diagnose, Therapie und Pflege sind mit zusätzlichem Aufwand verbunden und setzen besonderes Fachwissen und besondere kommunikative Kompetenzen bei Pflegenden wie Ärzten voraus. Zwischen den konkret zu behandelnden Symptomen können dabei mitunter komplexe Wechselwirkungen auftreten, die die Diagnose, Behandlung und Pflege zusätzlich erschweren. ${ }^{175}$

Die Ärztekammer Berlin plädiert in diesem Zusammenhang für ein „barrierefreies Krankenhaus“. Die baulichen Voraussetzungen sowie die Ausstattung mit technischen Hilfsmitteln entsprächen zwar in der Mehrzahl der Häuser weitgehend den Anforderungen an die Barrierefreiheit, es mangele aber an einer barrierefreien Kommunikation und an den Hilfssystemen, die vor allem Menschen mit Sinnesbehinderungen ein Höchstmaß an Unabhängigkeit sowie die Wahrung ihrer Eigenständigkeit und Selbstbestimmung während des Aufenthaltes im Krankenhaus ermöglichen.

Von den Behindertenfachverbänden wird zur Verbesserung der Versorgung von Menschen mit Behinderung im Krankenhaus der Abschluss einer Zielvereinbarung nach $₫ 5$ des Bundesgleichstellungsgesetzes zwischen der Deutschen Krankenhausgesellschaft und den Behindertenverbänden vorgeschlagen. ${ }^{17}$ Damit sollen der Mangel an verbindlichen

\footnotetext{
175 Von diesen Ausgangsbedingungen ließ sich der Deutsche Ethikrat bereits bei seinem Forum Bioethik „Menschen mit Behinderung - Herausforderungen für das Krankenhaus" 2014 in München leiten. Teilnehmer erhoben Forderungen nach einer Verbesserung der Versorgung von Menschen mit Behinderung in den Krankenhäusern, aber auch dem Aufbau spezialisierter Zentren, die nicht nur komplexe Diagnosestellungen und Behandlungen sicherstellen, sondern auch Beratungsfunktion für die Krankenhäuser übernehmen können. Siehe http://www.ethikrat.org/veranstaltungen/ forum-bioethik/menschen-mit-behinderung [22.02.2016].

176 Vgl. unter anderem Roser/Budroni/Schnepp 2011.
} 
Beschreibungen organisatorischer Maßnahmen und Abläufe für die Versorgung von Patienten mit schweren und mehrfachen Behinderungen im Krankenhaus behoben, eine bessere Aus- und Weiterbildung des ärztlichen, therapeutischen und pflegerischen Personals sichergestellt, verbindliche Qualitätsstandards durchgesetzt und ein auf die Bedarfe dieser Gruppe abgestelltes Aufnahme- und Überleitungsmanagement vereinbart werden. Großer Handlungsbedarf besteht im Bereich der zusätzlichen Finanzierung für die Versorgung von Menschen mit Behinderung im Krankenhaus und bei einer gesetzlichen Neuregelung der persönlichen Krankenhausassistenz, die bedarfsgerecht allen Menschen mit Behinderung ab einem bestimmten Assistenzbedarf bereitgestellt werden sollte. ${ }^{177}$

\subsubsection{Patienten mit Migrationshintergrund}

In Deutschland leben ca. 16,5 Millionen Menschen mit Migrationshintergrund, was einem Bevölkerungsanteil von 20,5 Prozent entspricht. ${ }^{178}$ In Ballungsgebieten kommt es häufig vor, dass 30 bis 50 Prozent der Patienten auf Krankenhausstationen Migrationshintergrund aufweisen. Aufgrund dieser demografischen Situation gehören interkulturelle Behandlungssituationen in zahlreichen deutschen Krankenhäusern zum Berufsalltag. Sprachliche und kulturelle Barrieren, aber auch unterschiedliche moralische Wertvorstellungen führen dabei

177 Nach dem Gesetz zur Regelung des Assistenzpflegebedarfs im Krankenhaus vom 30. Juli 2009 (BGBI. I, 2495) haben Menschen mit Behinderung Anspruch, ihre vertrauten Pflegekräfte als Pflegeassistenten mit ins Krankenhaus zu bringen, wenn sie ihre Pflegekräfte im sogenannten Arbeitgebermodell beschäftigen, was aber nur die allerwenigsten Menschen, die Pflege benötigen und Leistungen aus der Pflegeversicherung beziehen, tun. Die meisten der Betroffenen erhalten Pflegeleistungen als ambulante Sachleistung oder im Rahmen einer Heimunterbringung und fallen deshalb aus der bisherigen gesetzlichen Regelung heraus. Eine Gesetzesänderung, die auch die Behinderten- und Gleichstellungsbeauftragten der Länder anstreben, würde Menschen mit Behinderung, die Sachleistungen beziehen oder im Heim untergebracht sind, einbeziehen.

178 Statistisches Bundesamt 2014, 38. 
annähernd regelhaft $\mathrm{zu}$ unterschiedlichen Schwierigkeiten und ethischen Konflikten. Eine solche Situation beeinträchtigt nicht nur den Zugang zu einer adäquaten Gesundheitsversorgung im Krankenhaus, sondern stellt auch die Behandelnden im Krankenhaus vor neue Herausforderungen.

Sprachliche Barrieren spielen eine entscheidende Rolle bei der Fehl-, Unter- und Überversorgung von Patienten mit Migrationshintergrund im Krankenhaus. Oft sind Patienten aus anderen Kulturkreisen der deutschen Sprache nicht mächtig; professionelle Dolmetscher stehen nur in seltenen Fällen zur Verfügung. ${ }^{179}$ Zur Überwindung von Verständigungsschwierigkeiten werden häufig Zufallsdolmetscher herangezogen. Bei solchen Dolmetschertätigkeiten führt mangelnde Sprachkompetenz häufig zu Fehlübersetzungen, Auslassungen oder Zensur. Um mögliche Lücken in der Kommunikation zu kompensieren, werden häufig zusätzliche Untersuchungen durchgeführt, die bei adäquater Kommunikation vermeidbar wären und zusätzliche finanzielle und personelle Ressourcen erfordern. ${ }^{180}$ Diese Konstellationen erschweren nicht nur eine adäquate und qualitätsgesicherte Behandlung im Krankenhaus, sondern werfen zugleich gravierende ethische und rechtliche Probleme auf. Denn Kommunikationsdefizite dieser Art, die oftmals auch unbemerkt bleiben bzw. sich einer Kontrolle entziehen, behindern die erforderliche Patientenaufklärung und erlauben somit dem Patienten nicht, selbstbestimmt eine informierte Einwilligung zu erteilen.

Zusätzlich zu den Verständigungsschwierigkeiten beeinträchtigen auch kulturelle Barrieren den Zugang zu Gesundheitsleistungen im Krankenhaus und deren Inanspruchnahme. Unter kulturellen Barrieren sind zum einen Missverständnisse über Entscheidungen und Handlungen des Patienten zu verstehen, die von kulturellen Wertvorstellungen geprägt sind, den Behandelnden fremd sind und häufig wenig nachvollziehbar

179 Vgl. Barkowski 2008.

180 Bioethik-Kommission des Landes Rheinland-Pfalz 2010, 87. 
erscheinen. Bei der Durchführung medizinischer Maßnahmen können zum anderen ein kulturell geprägtes intensives Schamgefühl oder die Befolgung bestimmter religiöser Pflichten, etwa die Beachtung von Speisevorschriften, sowohl für Behandler als auch für Patienten eine Herausforderung darstellen. ${ }^{181}$

Unterschiedliche moralische Bewertungen der medizinischen Maßnahmen sind keine seltenen Erfahrungen in den Krankenhäusern wertpluraler Gesellschaften. Es gibt jedoch moralische Haltungen von Patienten aus anderen Kulturkreisen, die auf bestimmte kulturelle Wertüberzeugungen zurückzuführen sind. Beispiel hierfür sind etwa Entscheidungen in der Familie des Patienten, ob diesem die Diagnose einer bösartigen und unheilbaren Krebserkrankung mitgeteilt werden soll, die Forderung nach maximaler Therapie auch in medizinisch aussichtslosen Situationen unter Berufung auf religiöse Argumente oder die Einflussnahme traditionell geprägter hierarchischer Strukturen auf die Entscheidungsfindung, etwa wenn der Ehemann für die Ehefrau entscheidet. ${ }^{182}$ Solchen Forderungen liegen moralische Haltungen zugrunde, die der hiesigen Kultur fremd sind, oder ein unterschiedlicher Stellenwert der in der Medizinethik bekannten ethischen Prinzipien wie des Prinzips des Respekts vor der Patientenautonomie. Ethische Interessen- und Entscheidungskonflikte im Klinikalltag sind oft besonders komplex und deshalb eine Herausforderung für Krankenhausmitarbeiter. ${ }^{183}$

\subsubsection{Registrierte Flüchtlinge und Menschen ohne Aufenthaltsstatus}

Die hohe Anzahl von Flüchtlingen, die seit ca. einem Jahr nach Deutschland kommen, wirft unter anderem die Frage nach

181 Zuckerman et al. 2002; Ilkilic 2002; Ilkilic 2006; Sattar et al. 2004.

182 Vgl. Ilkilic 2008.

183 Vgl. Ilkilic 2014. 
den Bedingungen für die Behandlung dieser Menschen im Krankenhaus in Deutschland auf. Überdies halten sich nach Schätzungen in Deutschland mindestens 500.000 bis eine Million Menschen ohne gültige Aufenthaltspapiere (sogenannte sans papiers) auf, zu denen neben illegal in Deutschland befindlichen Menschen auch Flüchtlinge zu zählen sind, bevor sie registriert wurden. Wenngleich für die Behandlung dieser Patientengruppen im Prinzip Regularien ${ }^{184}$ bestehen, beziehen sich zahlreiche Fragen auf deren praktische Umsetzung. ${ }^{185}$ Dieses Problemfeld kann aufgrund seiner Komplexität, die nicht zuletzt auf die hohe Anzahl der betroffenen Personen zurückzuführen ist und zudem auch Fragen nach der Übereinstimmung völkerrechtlicher, verfassungsrechtlicher und sozialrechtlicher Grundlagen betrifft, im Rahmen der vorliegenden

184 Siehe dazu Zentrale Ethikkommission bei der Bundesärztekammer 2013b; Entschließung des 109. Deutschen Ärztetages 2006 (Drucksache VII-11): „Unzureichende medizinische Versorgung von Menschen ohne legalen Aufenthaltsstatus" (http://www.bundesaerztekammer.de/arzt2006/data/ anhang_a/topo7_11_E_END.pdf [02.03.2016]). Siehe zur Gesundheitsversorgung von Asylsuchenden (Untersuchung und Versorgung in Erstaufnahmeeinrichtungen, Versorgung und Behandlung von psychischen Erkrankungen und Traumata, Bedarf an qualifiziertem Personal, Einbeziehung sprachlicher und interkultureller Aspekte, Verbesserung der Daten und Forschungslage) die gemeinsame Kurz-Stellungnahme „Zur Gesundheitsversorgung von Asylsuchenden“ der Nationalen Akademie der Wissenschaften Leopoldina, der Deutschen Akademie der Technikwissenschaften und der Union der deutschen Akademien der Wissenschaften von 2015.

185 In diesem Zusammenhang wären etwa die Fragen zu behandeln, inwieweit es vor dem Hintergrund völkerrechtlicher Verpflichtungen (zum Beispiel dem Internationalen Pakt über wirtschaftliche, soziale und kulturelle Rechte von 1966 oder der UN-Resolution 48/104 über die Beseitigung der Gewalt gegen Frauen von 1993) zu rechtfertigen ist, dass im Asylbewerberleistungsgesetz $(\$ \S 4,6)$ die Behandlung von registrierten Flüchtlingen innerhalb der ersten 15 Monate ihres Aufenthalts auf akute Erkrankungen und Schmerzzustände beschränkt wird, während zum Beispiel die bei vielen dieser Patienten vordringliche Behandlung einer Kriegstraumatisierung nicht ermöglicht wird, ferner, dass der Zugang dieser Personen zu einer ärztlichen Versorgung in manchen Bundesländern durch die in der Regel medizinisch nicht fachkundigen Heimleiter der Aufnahmestätten reguliert wird. Bei Menschen ohne gültige Aufenthaltspapiere wäre zu prüfen, wie eine Krankenhausbehandlung ermöglicht werden kann, die im Sinne des verlängerten Geheimnisschutzes die ärztliche Schweigepflicht wahrt, zum Beispiel durch das in einigen Ländern eingeführte Instrument eines anonymen Krankenscheins. 
Stellungnahme nur benannt, jedoch nicht ausführlicher behandelt werden.

\subsection{Ressourcenallokation}

Es ist unstrittig, dass die Gesellschaft ein Interesse an einer allgemein zugänglichen, qualitativ hochwertigen medizinischen Versorgung auf dem aktuellen Stand von Medizin, Biowissenschaft und Technik hat. Dies ist nicht nur im Interesse der Patienten, sondern auch im Interesse der akut nicht von Krankheit Betroffenen. Denn diese können sicher sein, im Fall einer Erkrankung schnellstmöglich eine qualitativ hochwertige medizinische Versorgung in Anspruch nehmen zu können. Dieser Bedarf tritt vielfach unvorhergesehen und akut ein, sodass sogenannte „Reservekapazitäten“ vorgehalten werden müssen. Stationäre Einrichtungen haben somit den Charakter eines Optionsguts, das heißt, bereits die Existenz einer stationären Versorgung stiftet den Mitgliedern der Gesellschaft einen Nutzen. Dieser Optionsgutcharakter tritt vor allem im Bereich der Notfallversorgung, der Katastrophenversorgung sowie in der intensivmedizinischen Versorgung zutage.

Neben dem unbestrittenen Nutzen medizinischer Leistungen für die Gesellschaft muss berücksichtigt werden, dass die öffentlichen Ressourcen, die in den stationären Sektor investiert werden, hoch und begrenzt sind. Damit stellen sich automatisch Fragen der Mittelverteilung sowie der Effizienz und Effektivität. Durch andere Strukturen und organisatorische Maßnahmen lassen sich aber auch Ressourcen gewinnen und umverteilen. Deshalb muss eine gesellschaftliche Entscheidung darüber getroffen werden, in welchem Umfang Mittel für die stationäre Versorgung verausgabt werden. Im stationären Sektor erfolgt die Festlegung der Gesamtkapazitäten im Rahmen der Bedarfsplanung durch die Bundesländer und damit im Rahmen des politischen Willensbildungsprozesses. Die vorgehaltene Zahl der Betten hat immer auch Einfluss auf die 
Menge der behandelten Fälle und damit Leistungen. ${ }^{186}$ Für die Frage, ob eine bestehende Einrichtung unter den gegebenen Rahmenbedingungen wirtschaftlich betrieben werden kann und wie viele Fälle und welche Leistungen sie dafür erbringen muss, ist neben der Größe und Struktur des stationären Sektors jedoch vor allem dessen Finanzierung von entscheidender Bedeutung.

Die Entscheidung über die wirtschaftlich effiziente und sozial gerechte Zuteilung knapper medizinischer Ressourcen erfolgt auf verschiedenen Stufen. ${ }^{187}$ Auf deren erster ist darüber zu befinden, wie hoch die Mittel für den Gesundheitssektor insgesamt bemessen werden, das heißt, welcher Anteil des Volkseinkommens für die Gesundheitsaufgaben aufgewendet wird.

Wird eine derartige monetäre Obergrenze für das Gesundheitssystem festgelegt - eine gewisse Beschränkung auf der gesamtwirtschaftlichen Ebene enthält der Grundsatz der Beitragssatzstabilität ( $\$ 71$ SGB V) und das Wirtschaftlichkeitsgebot ( $\$ 12$ SGB V) -, zieht das notwendigerweise Allokationsentscheidungen auf den nachgelagerten Ebenen nach sich. Wenn auf allen Ebenen die begrenzten Mittel effizient und effektiv eingesetzt werden sollen, dient dies dem Zweck, Unter-, Über-, und Fehlversorgung zu vermeiden. Hierfür kann die Finanzierungsform des stationären Sektors einen wesentlichen Beitrag leisten.

Auch der Zusammenhang zwischen Investitionen in Gebäude und technische Infrastruktur (eingesetztes Kapital) und Betriebskosten ist für die jeweilige Folgenbetrachtung von Bedeutung. Beispielsweise mag eine neue Gebäudestruktur

186 Vgl. unter anderem das sogenannte Roemersche Gesetz : „in an insured population, a hospital bed built is a filled bed“" (Shain/Roemer 1959, 71); siehe auch Sauerland 2002, $84 \mathrm{ff}$.

187 Ein Beispiel für eine ineffiziente Aufteilung zwischen den Sektoren des Gesundheitswesens ist die Verlagerung der Versorgung durch niedergelassene Ärzte auf die Notfallversorgung durch Krankenhäuser. Ein Indikator dafür, dass eine solche Verlagerung stattfindet, ist der starke Anstieg der ambulanten Notfallversorgung durch die Krankenhäuser. 
geringere Energiekosten verursachen oder mögen Mitarbeiter bei funktionaler Anordnung und sinnvollen Bereichsgrößen ohne zusätzliche Belastung mehr Patienten versorgen können als bei langen Transportwegen oder zu geringen Stationsgrößen. Private Krankenhausträger setzen mehr Kapital („Sachanlagevermögen") pro Euro Umsatz ein als die öffentlichen und freigemeinnützigen Träger. 71 Cent bei den privaten stehen 70 Cent bei öffentlichen und 58 Cent bei freigemeinnützigen Trägern gegenüber. ${ }^{188}$ Wenn damit bei öffentlichen und freigemeinnützigen Trägern weniger Mittel zur Verfügung stehen, um durch entsprechende Investitionen zum Beispiel prozessbehindernde Gebäudestrukturen zu verändern, veraltete Medizintechnik zu ersetzen oder hohe Energie- und Instandhaltungskosten zu reduzieren, besteht ein höherer Bedarf, personelle und sachliche Ressourcen einzusetzen. ${ }^{189}$

\subsubsection{Duale Finanzierung im stationären Sektor}

Die Krankenhausfinanzierung im dualen Modell (siehe 2.2) wird seit Längerem kritisiert. Ursprünglich wurde seitens der Krankenkassen problematisiert, diese Form der Finanzierung schaffe Überkapazitäten im stationären Sektor. Hintergrund dieser Kritik ist, dass die Bundesländer nur Investitionen zu leisten haben, während die Kassen die laufenden Betriebskosten abdecken müssen. Aufgrund der Mittelknappheit in den öffentlichen Haushalten ist seit einiger Zeit jedoch zu beobachten, dass die Krankenhausinvestitionen hinter der Entwicklung der Ausgaben im Gesundheitswesen zurückbleiben.

$188 \mathrm{Vgl}$. Augurzky et al. 2015, 162.

189 Für die Kalkulation der DRGs melden Krankenhäuser zurzeit auf freiwilliger Basis ihre Kosten- und Leistungsdaten an das InEK. Bisher nehmen die privaten Krankenhausketten nicht an der Kalkulation teil. Per Gesetz soll das InEK ermächtigt werden, Häuser zur Teilnahme aufzufordern. Sollten auf diesem Weg Daten privater Krankenhausketten in die Kalkulation der DRGs einfließen, ist der beschriebene Zusammenhang zwischen eingesetztem Kapital, also Investitionen, und Betriebskosten zu berücksichtigen. 
So haben sich die Ausgaben der gesetzlichen Krankenversicherung im Zeitraum 2002 bis 2013 um etwa 40 Prozent erhöht, während die Länderinvestitionen in den stationären Sektor im gleichen Zeitraum um etwa 15 Prozent gesunken sind. ${ }^{190}$ Mittlerweile spricht man von einem Investitionsstau im Krankenhausbereich, der auf mehrere Milliarden Euro geschätzt wird (siehe 2.2) Die Umstellung etwa auf ein monistisches System einer Finanzierung „aus einer Hand“, wie es etwa der Sachverständigenrat zur Begutachtung der Entwicklung im Gesundheitswesen empfiehlt ${ }^{191}$, ist bisher an der Frage gescheitert, wie und in welcher Höhe - auch unter Berücksichtigung des Investitionsstaus - die Einrichtungen die zusätzlich erforderlichen finanziellen Mittel erhalten. ${ }^{192}$ Zudem würde sich in einem monistischen System ${ }^{193}$ der Einfluss der Bundesländer reduzieren, die aber weiterhin den Sicherstellungsauftrag für die stationäre Versorgung hätten. Man kann aber natürlich die Frage des Investitionsstaus auch so adressieren, dass man fragt, ob die Zahl der vorhandenen Krankenhäuser notwendig ist. Der Sachverständigenrat hat mehrfach die Frage des planvollen Abbaus von Überkapazitäten mit der Forderung nach einer notwendigen Reform der Investitionsfinanzierung verbunden. ${ }^{194}$ In diesem Fall könnte das begrenzte Investitionsbudget auf eine geringere Zahl von verbliebenen Krankenhäusern effizienter verteilt werden. Vergleichszahlen mit volkswirtschaftlich auf ähnlichem Level wie Deutschland anzusiedelnden Ländern wie die Niederlande, Dänemark oder Schweden legen die Vermutung nahe, dass auch dieser Pfad

\footnotetext{
190 Siehe http://aok-bv.de/gesundheit/versorgungsbereiche/krankenhaus/ index_11287.html [23.02.2016].

191 Sachverständigenrat zur Begutachtung der Entwicklung im Gesundheitswesen 2014, Ziffer 512; Sachverständigenrat zur Begutachtung der Entwicklung im Gesundheitswesen 2012, Ziffer 337.

192 Vgl. Preusker 2010, 302.

193 Vgl. Fußnote 57.

194 Sachverständigenrat zur Begutachtung der Entwicklung im Gesundheitswesen 2014, Ziffer 264.
} 
der Effizienzsteigerung zur Verbesserung der Krankenhausinfrastruktur bedacht werden sollte. ${ }^{195}$

Neben der generellen Unterfinanzierung bei den Investitionen besteht das zusätzliche Problem erheblicher Differenzen zwischen den Investitionsleistungen der einzelnen Bundesländer. Dies hat bereits zu einer deutlich unterschiedlichen Versorgung der Bevölkerung mit stationären Leistungen in den einzelnen Bundesländern geführt. Damit trägt die bisherige Krankenhausfinanzierung zu einer Ungleichheit in einem Bereich bei, der für die Lebenslage der Betroffenen von herausragender Bedeutung ist. Obgleich die stationäre Versorgung in Deutschland im internationalen Vergleich quantitativ als sehr gut anzusehen ist (siehe 2.1), kann mittel- bis langfristig nicht ausgeschlossen werden, dass insbesondere in dünn besiedelten ländlichen Regionen eine Unterversorgung im stationären Bereich auftritt. Der Sachverständigenrat zur Begutachtung der Entwicklung im Gesundheitswesen hat dazu empfohlen, neben einer Festlegung der Fachrichtungen, die für eine flächendeckende Grundversorgung unabdingbar sind, entsprechende Mindesterreichbarkeitskriterien (vorzugsweise eher Wegezeiten als Entfernungen) zu definieren und Krankenhäuser, die dann für die Versorgung dünn besiedelter Gebiete unverzichtbar sind, durch einen Sicherstellungszuschlag zu stützen. ${ }^{196}$

195 Der Ländervergleich vor allem zu volkswirtschaftlich ähnlich strukturierten Ländern in Europa zeigt, dass Deutschland trotz rückläufiger Kapazitäten noch immer über überdurchschnittlich viele Betten und Krankenhäuser (mit zum Teil fragwürdiger Ausstattung) verfügt. Während nach 2014 ermittelten Daten die Niederlande 4,7 Krankenhausbetten pro Tausend Einwohner und Dänemark sogar nur 3,4 Betten vorhielten, waren es in Deutschland 8,3 pro Tausend Einwohner (http://www.indexmundi.com/g/ r.aspx?v=2227\&l=de [23.02.2016]). Auch dem erwähnten Bettenabbau, der in Deutschland in den letzten beiden Jahrzehnten 15 Prozent betrug, steht ein Abbau von 27 Prozent in den alten EU-15-Ländern gegenüber. Mit Blick auf die Verweildauer und Krankenhausfälle liegt Deutschland über dem Schnitt der alten EU-15-Länder. Zu diesem und weiterem Zahlenmaterial, nach dem der internationale Vergleich auch Rückfragen an die Ergebnisqualität deutscher Krankenhäuer nahelegt, vgl. Geissler/Busse 2015.

196 Sachverständigenrat zur Begutachtung der Entwicklung im Gesundheitswesen 2014, Ziffer 263. 
In diesem Zusammenhang stellt sich auch die Frage, ob die primär an der Qualität der Behandlung zu orientierende Versorgungsplanung eher den derzeitigen Zielen einer standortbasierten Planung oder den Zielen einer erreichbarkeitsbasierten ${ }^{197}$ Planung folgen sollte, wobei patientenwohlorientierte Argumente für beide Zielrichtungen $\mathrm{zu}$ finden sind. Gleichzeitig stellt sich die Frage, ob die Krankenhausplanung und die Finanzierung der Einrichtungen stärker nach bundeseinheitlichen Kriterien erfolgen sollten und die Finanzierung länderübergreifend geregelt werden sollte.

\subsubsection{Trägerstruktur im stationären Sektor}

Seit einigen Jahren ist in Deutschland ein signifikanter Wandel in der Trägerstruktur der Krankenhäuser festzustellen (siehe 2.1), wobei sich der Sektor der privatwirtschaftlichen Einrichtungen von einem „Nischenprodukt“ zu einem bedeutenden Träger der Gesundheitsversorgung entwickelt.

Empirische Untersuchungen zeigen, dass private Einrichtungen ein höheres Maß an Wirtschaftlichkeit aufweisen als kommunale und freigemeinnützige Einrichtungen. Hierfür können verschiedene Faktoren ursächlich sein. Neben einem höheren gezielten Kapitaleinsatz und einer höheren Entscheidungsgeschwindigkeit der Einrichtung werden ein erfolgsorientiertes Management, geringere Personalkosten sowie bessere Möglichkeiten des Outsourcings bestimmter Leistungen genannt. ${ }^{198}$ Privaten Anbietern wird häufig vorgeworfen, sie

197 Gerade wenn man auf die Mittel Bettenabbau und Krankenhausschließungen setzt, kann die womöglich längere Fahrtstrecke (gerade in der Notfallversorgung) durch mehr Kapazitäten in der Luftrettung unter dem Gesichtspunkt medizinischer Effektivität wie gesundheitsökonomischer Effizienz ausgeglichen werden.

198 Augurzky/Beivers/Gülker 2012, 13 f., 21-26, 38-42. 
würden „Rosinenpickerei“"199 betreiben, das heißt sich auf die lukrativen Fälle konzentrieren, während die kostenintensiven Bereiche von ihnen nicht bedient werden. ${ }^{200}$

Inwieweit dieser Vorwurf insgesamt berechtigt ist, ist in der Literatur umstritten. ${ }^{201}$ So zeigt eine Studie über Krankenhäuser in privater Trägerschaft, dass der Schweregrad der Erkrankung sowie das Alter der Patienten in privaten Krankenhäusern nicht geringer ist als in öffentlichen oder freigemeinnützigen Einrichtungen. ${ }^{202}$ Dieser Umstand ist für sich genommen allerdings noch kein Beleg dafür, dass private Einrichtungen die gleiche Breitenversorgung übernehmen wie öffentliche Häuser. Gegen die Annahme einer vergleichbaren Breitenversorgung durch private Träger spricht zum Beispiel, dass private Einrichtungen in deutlich geringerem Maße kostenintensive Notfallambulanzen anbieten als öffentliche oder freigemeinnützige Träger. Die Notfall- und Akutmedizin stellt aber nicht nur einen kostenintensiven und wirtschaftlich unrentablen Bereich der (teil)stationären Versorgung dar, sondern ist für die Gesundheitsversorgung der Bevölkerung wesentlich und unverzichtbar.

Grundsätzlich ist eine Spezialisierung von Einrichtungen auf bestimmte Therapien medizinisch und wirtschaftlich sinnvoll. Sie können insbesondere bei planbaren operativen Eingriffen auf dem aktuellen Stand der Technik und des medizinischen Wissens arbeiten. Insbesondere für kleinere

199 Vgl. Bräutigam/Kruse 1992. Bei privaten Krankenhäusern ist zwischen Klinikketten und einzelnen Häusern zu unterscheiden. Dass einzelne Häuser eine wirtschaftlich motivierte Selektionsstrategie verfolgen, lässt sich nicht ausschließen. Dies gilt umgekehrt aber auch für Häuser in anderer Trägerschaft.

200 Für andere Häuser im Umfeld bedeutet dies, dass sie im Gegenzug schwerpunktmäßig die Notfallversorgung bzw. die Versorgung der vermeintlich nicht wirtschaftlich attraktiven Leistungsfelder übernehmen, um die Versorgung sicherzustellen. Damit sind hohe Vorhaltekosten und geringere Skaleneffekte mit entsprechend geringerer Effizienz verbunden.

201 Datenanalysen zu den versorgten Patienten im Hinblick auf das Alter und die Versicherungsart (gesetzlich versus privat) zeigen bezogen auf die Gesamtheit privater Anbieter keine Auffälligkeiten (Augurzky/Beivers/Gülker 2012, 28).

202 Augurzky/Beivers/Gülker 2012, 28-31. 
Einrichtungen ist die Spezialisierung eine adäquate Strategie, um sich in einem zunehmend kompetitiven Umfeld zu behaupten. Dies gilt unabhängig von der Trägerschaft. Auch in Verbünden in öffentlicher oder freigemeinnütziger Trägerschaft sind Konzentrationsstrategien zu beobachten, die ebenfalls den Rahmen für eine qualitativ hochwertige Versorgung schaffen.

Eine zunehmende Spezialisierung in der Krankenhauslandschaft erscheint gesundheitspolitisch wie ethisch unproblematisch, solange die erforderlichen Vorhaltekapazitäten für die kostenintensiven Intensiv- und Notfallstationen, die zunehmend zurückgedrängten Fächer wie Gynäkologie, Geburtshilfe und Kindermedizin sowie die Behandlung seltener Erkrankungen sichergestellt sind und durch die öffentliche Hand ausreichend finanziell unterstützt werden. In vielen $\mathrm{Re}$ gionen stellt allerdings die flächendeckende, respektive schnell erreichbare Sicherstellung und die adäquate Finanzierung von Notfallkapazitäten mittlerweile ein erhebliches Problem dar.

\subsubsection{Anreize und Fehlanreize des DRG-Systems}

Im bestehenden DRG-Abrechnungssystem arbeiten jene Häuser wirtschaftlich, deren Durchschnittskosten für einen Behandlungsfall unter der Vergütung der diagnoseorientierten Fallpauschalen liegen. Diese Bedingung wird insbesondere von Einrichtungen erfüllt, die sich auf bestimmte Krankheitsbilder und therapeutische Verfahren spezialisiert haben. Diese Einrichtungen können ihre Fallzahlen in diesen Bereichen entsprechend erhöhen und dadurch in hohem Maße rentabel arbeiten. Grundsätzlich werden durch dieses System die knappen öffentlichen Mittel jener Verwendung zugeführt, die den größten medizinischen Nutzen stiftet. Allerdings gibt es Behandlungsfelder, in denen die DRGs erfahrungsgemäß unzureichend sind, um die anfallenden Durchschnittskosten abzudecken. Hierzu gehören unter anderem die kinder- und 
jugendmedizinischen Fachabteilungen als auch die Versorgung multimorbider alter Patienten (siehe 4.5.1 bis 4.5.4).

Ziel muss es sein, für alle Behandlungsfälle kostenadäquate Finanzierungen sicherzustellen, wobei auch zu prüfen ist, unter welchen Bedingungen Fälle, Fallgruppen oder Patientengruppen außerhalb der Fallpauschalen zu finanzieren sind.

Ein am Patientenwohl orientiertes Vergütungssystem kann im Wesentlichen auf Fallpauschalen basieren, sofern zu erwarten ist, dass bestimmte typische Behandlungssituationen unter Berücksichtigung der oben genannten Kriterien hinreichend durch standardisierte Variablen beschrieben und diese in pauschalierte Vergütungen umgesetzt werden können. Eine Standardisierung solcher Situationen spart Aufwand und Kosten. Gleichwohl kann jede klinische Behandlung dazu führen, dass Ressourcen erforderlich werden, die weit über die vorgesehenen Standards hinausgehen. Zudem finden innovative Behandlungen insbesondere in der Hochleistungsmedizin teilweise erst nach jahrelangen Vorbereitungen Eingang ins DRG-System. Dies gilt insbesondere bei multimorbiden alten Patienten, bei Patienten mit seltenen Erkrankungen und in der Notfallversorgung. Solche Situationen ihrerseits zu standardisieren, erscheint wegen der Notwendigkeit einer weit aufgefächerten Differenzierung wenig sinnvoll.

Eine Lösungsmöglichkeit könnte darin bestehen, aufbauend auf der Regelung des $\$ 17 \mathrm{~b}$ Abs. 1 Satz 10 KHG zu den „besonderen Einrichtungen“, die Möglichkeit auszuweiten, krankenhausindividuelle Entgeltsätze für Fallgruppen zu vereinbaren, bei denen die komplexe individuelle Behandlungssituation auf der Basis tatsächlich entstandener Kosten beglichen wird. Mit einem fallpauschalenbasierten und einem parallel dazu individualisierten Vergütungssystem würden auch kostenintensive notwendige Vorhaltungen, etwa 
die Unterhaltung von Notfallambulanzen ${ }^{203}$ im ländlichen Raum, vom fallbasierten Abrechnungssystem entkoppelt werden können. Dabei müssen allerdings klare Kriterien für die Vereinbarungsmöglichkeit solcher krankenhausindividuellen Entgeltsätze definiert werden, einschließlich einer Begrenzung auf Indikationen und Diagnosegruppen anhand von extern überprüfbaren Begründungen. Damit soll gesichert werden, dass die beabsichtigten Einspareffekte des DRG-Systems nicht ausgehebelt werden. Ein solches System könnte die Bereitschaft fördern, auch Patienten mit komplexen und ressourcenintensiven Vorerkrankungen (zum Beispiel Demenz, Infektionen bzw. Besiedlung mit multiresistenten Keimen) in den Krankenhäusern zu behandeln.

Als überprüfungsbedürftig kann das bisher im DRG-Fallpauschalensystem vorrangig zugrunde gelegte Vergütungskriterium der erbrachten ärztlichen Leistungen angesehen werden, das, gemessen etwa an den Ausweitungen von Fallzahlen und verkürzten Verweildauern, durchaus Anreize zu ethisch problematischem Handeln setzen kann. So hebt das Vergütungssystem stark auf das Handeln und Anwenden von aktiven Maßnahmen ab, wodurch auch Anreize für überflüssige, doppelte und somit unnötige Maßnahmen entstehen können, die den Patienten zusätzlich belasten. Eine vom GKV-Spitzenverband, dem Verband der Privaten Krankenversicherung und der Deutschen Krankenhausgesellschaft in Auftrag gegebene und 2014 vorgelegte Studie konnte allerdings keine eindeutigen Belege für oder gegen eine Mengenausweitung in den Kliniken allein aus ökonomischer Motivation liefern. $\mathrm{Zu}$ viele Effekte hätten Einfluss auf die Leistungsentwicklung: Morbidität

203 Die ambulante Notfallversorgung der gesetzlich Versicherten wird nicht durch das DRG-System bezahlt, sondern über fallbezogene Honorare nach dem einheitlichen Bewertungsmaßstab der Kassenärztlichen Vereinigung vergütet. Dennoch besteht auch hier das Problem einer möglichen Unterfinanzierung, da die Pauschalen für ambulante Notfälle nicht kostendeckend sind und durch andere Erträge quersubventioniert werden müssen. Hier liegen die durchschnittlichen Behandlungskosten zum Teil deutlich über den entsprechenden Vergütungssätzen (vgl. Haas et al. 2015, 11 f., 38 f., 48 f., 61 f.). 
und Mortalität, der medizinische Fortschritt, die Erwartungen der Patienten, veränderte Lebensgewohnheiten oder auch sich ändernde Wechselwirkungen mit der ambulanten Versorgung und der demografische Effekt. Allerdings fassen die Autoren die Ergebnisse so zusammen, dass zwar bei akuten Leistungen, wie akutem Herzinfarkt, das Aufnahmeverhalten nicht verändert wurde, bei anderen akuten Leistungen, wie zum Beispiel Gefäßerkrankungen, die veränderten Fallzahlen auf ein verändertes Kodierverhalten oder andere Behandlungspfade zurückzuführen seien, bei planbaren Leistungen oder Leistungen in Bereichen unklarer Evidenzlage aber, wie zum Beispiel Spondylose, sich die Fallzahlen als Folge der DRGs verändert hätten. ${ }^{204}$ Die Autoren betonen allerdings, dass die ihnen zur Verfügung stehenden Daten keinen Schluss darüber zulassen, ob die Fallzahlveränderungen medizinisch indiziert waren oder nicht. Die Studie stellt auch einen Zusammenhang zwischen steigenden Kosten bei gleichbleibenden DRGs und sinkenden Fallzahlen fest ${ }^{205}$ sowie sinkende Fallzahlen bei fallenden DRG-Gewichten ohne Zusatzentgelte und Fallzahlsteigerungen bei der Möglichkeit von Zusatzentgelten ${ }^{206}$. Die Pendeleffekte zwischen DRG- und Fallzahlentwicklung scheinen zumindest für bestimmte Diagnosebereiche nachweisbar, wenngleich sich die Frage, ob diese Entwicklungen über das medizinisch Gebotene oder Notwendige hinausgehen, nicht beantworten lässt.

Unabhängig vom Einfluss der jeweiligen Form der Vergütung wird in der Ärzteschaft zunehmend die Vermeidung

204 Schreyögg et al. 2014, 13.

205 Ebd., 80.

206 Ebd., 93. 
medizinisch nicht erforderlicher Maßnahmen diskutiert. ${ }^{207}$ In diesem Sinne hat auch die Arbeitsgemeinschaft der Wissenschaftlichen Medizinischen Fachgesellschaften 2015 eine Diskussion „Gemeinsam klug entscheiden“ gestartet, die sich der internationalen Initiative "Choosing wisely" anschließt und zum Ziel hat, für unnötige und sogar schädliche Leistungen „Don’t do-Empfehlungen“ zu erarbeiten. ${ }^{208}$

Vor diesem Hintergrund sollte die Vergütung der medizinischen Diagnostik und der Beobachtung eines Patienten ohne Durchführung einer nachfolgenden Behandlungsleistung im DRG-Katalog stärker akzentuiert, zielorientiert und besser honoriert werden. Dies könnte durch die Einführung einer eigenen und ausreichend finanzierten Prozedur „Beobachtung“ geschehen. Auf diese Weise würde etwa bei einer fraglichen medizinischen Indikation eine ärztliche Entscheidung zum beobachtenden Zuwarten einer ökonomischen Sanktionierung enthoben.

Auch dem Phänomen der Segmentierung der Behandlung multimorbider Patienten in mehrere auf jeweils eine Diagnose gestützte, einzeln abrechnungsfähige Krankenhausaufenthalte müsste durch Veränderungen innerhalb des DRG-Systems begegnet werden. Denkbar sind Paketlösungen, bei denen im individuellen Fall mehrere parallele Diagnosen zu einer übergeordneten DRG zusammengezogen werden können. Auch sollte eine am Patientenwohl orientierte Vergütungsstruktur im Bedarfsfall zu der stringenten Vermittlung eines Patienten in eine besser geeignete und spezialisierte Einrichtung führen,

207 So äußert sich Michael Hallek von der Deutschen Gesellschaft für Innere Medizin in diesem Zusammenhang in einer Pressemitteilung vom 24. Februar 2015: „Viele medizinische Eingriffe bedeuten auch eine Belastung für den Patienten. [...] Als Ärzte ist es nicht nur unsere Pflicht zu behandeln, sondern auch Behandlungen zu unterlassen, wenn sie dem Patienten nichts nützen oder ihm sogar schaden könnten“" (https://idw-online.de/de/news626239 [28.09.2015]). Außerdem heißt es in einer Pressemitteilung der Deutschen Gesellschaft für Innere Medizin vom 9. Juli 2015: „Leistungen, die erwiesenermaßen keinen Nutzen für den Patienten bringen sollten identifiziert und unterlassen werden“ (https://idw-online.de/de/news634544 [28.09.2015]). 208 Richter-Kuhlmann 2015. 
auch wenn ein Teil der Behandlung im abgebenden Haus durchgeführt werden könnte, was durch eine Bindung der einschlägigen DRGs an bestimmte Qualifikationen und Ausstattungsniveaus von Einrichtungen für die definierten Fallgruppen erreicht werden könnte. Eine Vergütungsstruktur, die eine weitere Reduzierung von Verweildauern auf einen kritisch kurzen Zeitraum und die Ausweitung von Fallzahlen fördert, geht in die falsche Richtung. Ebenso darf eine Verlängerung des stationären Aufenthaltes mit dem Ziel einer abschlagsfreien DRG-Vergütung und über die medizinische Notwendigkeit hinaus nicht erfolgen.

Ein weiteres Problem, das insbesondere Universitätskliniken und Einrichtungen mit flächendeckendem Versorgungsauftrag betrifft, stellt die Versorgung seltener Erkrankungen dar. Diese Einrichtungen können sich aufgrund ihres Versorgungsauftrags nicht aus unrentablen Bereichen zurückziehen und sind verpflichtet, auch Versorgungsleistungen für seltene Erkrankungsfälle zur Verfügung zu stellen. In Fällen, in denen Krankenhäuser - seien es Häuser der Maximalversorgung oder Häuser anderer Versorgungsstufen - die Versorgung seltener Erkrankungen übernehmen, ist zu prüfen, ob die Finanzierung dieser Einrichtungen neben einem preisgesteuerten System um eine entsprechende Grundfinanzierung der Vorhaltekosten ergänzt werden muss. Dies könnte beispielsweise durch entsprechende Sicherstellungszuschläge geschehen. 


\subsection{Qualitätssicherung und Dokumentation}

Zentrales Instrument der externen Qualitätssicherung im Krankenhaus ist der Qualitätsreport, den bisher das AQUAInstitut im Auftrag des G-BA erstellt. ${ }^{209}$ Der jüngste Bericht stammt aus dem Jahr 2013. Sowohl in der Richtlinie des G-BA als auch im Qualitätsreport wird unter anderem dargelegt, in welcher Form in einem „strukturierten Dialog“ mit negativen Abweichungen eines Krankenhauses hinsichtlich definierter medizinischer Parameter verfahren wird.

Die externe Qualitätssicherung im Krankenhaus ist mit dem Vorgehen im SGB XI und der dortigen Rolle des Medizinischen Dienstes der Krankenversicherung bei Qualitätskontrollen in vollstationären Pflegeeinrichtungen nicht vergleichbar, da bei den Kriterien des Qualitätsmanagements im Klinikbereich wissenschaftlich begründete Outcome-Kriterien existieren. Die Bewertungsfaktoren, die diesen Kriterien zugrunde liegen, werden über diverse medizinische Fachgesellschaften definiert und eingebracht.

Keine der beteiligten medizinischen Fachgesellschaften hat bisher Einwände gegen den AQUA-Qualitätsreport erhoben, sodass man eigentlich denken sollte, dass auch aus dem Klinikbereich, also von den zu Prüfenden, keinerlei Einwände oder Beschwerden erhoben werden. Jedoch wird gerade vonseiten der Kliniken und vielen Ärzten erhebliche Kritik geäußert. So wird unter anderem bemängelt, dass es sich um eine auf numerisch erfassbare Kriterien beschränkte Erhebung handele, bei der „weiche“ Faktoren wie die subjektive Zufriedenheit der Patienten kaum eine Rolle spiele. Die Pflege werde überhaupt nicht angemessen erfasst. Von derzeit 30 Leistungsbereichen

209 Nach $\S 137$ b SGB V ist der G-BA für die konzeptionelle Umsetzung der Qualitätssicherungsverpflichtungen von Leistungserbringern des Gesundheitswesens zuständig. Siehe hierzu die Richtlinie über Maßnahmen der Qualitätssicherung in Krankenhäusern vom 15. August 2006 (BAnz., 6361), zuletzt geändert am 16. April 2015 (BAnz. AT 06.08.2015 B2). 
betreffe nur einer die Pflege, und dieser ausschließlich die Dekubitusprophylaxe. Des Weiteren wird moniert, dass die Outcome-Erfolgskriterien für die Leistungsbereiche, die von einigen Krankenkassen veröffentlicht werden, zu vorschnellen Vergleichen zwischen den Kliniken führten, ohne die jeweiligen Ausgangsbedingungen (zum Beispiel Zusammensetzung der Patienten) mit einzubeziehen. Vielfache Beschwerden gibt es auch zu dem erheblichen Zeitaufwand, der mit den Erhebungen verbunden ist. Auffallend ist, dass diese Einwände offensichtlich vonseiten der Fachgesellschaften und Verbände nicht getragen und nicht an das AQUA-Institut weitergegeben werden.

Vor dem Hintergrund, dass das Anliegen der Qualitätssicherung (Steigerung der Qualität, Vergleichbarkeit und Erhöhung der Transparenz für die Nutzer) unstrittig ist, wird im neuen Krankenhausstrukturgesetz die Qualitätssicherung zu einem Bestandteil der Krankenhausplanung und Ressourcenzumessung erklärt. So werden $\mathrm{Zu}$ - und Abschläge für gute oder unzureichende Qualität eingeführt, womit sich die Frage der Parameter für die Feststellung von Qualität verschärft stellen wird.

Das AQUA-Institut hat die schon seit Längerem bestehende Kritik am bisherigen Verfahren aufgegriffen und Verbesserung der Kommunikation zwischen den Leistungserbringern und den Prüfinstanzen vorgeschlagen, um die Einrichtungsvergleiche fair und aussagekräftig zu gestalten. Des Weiteren sollten verstärkt Längsschnittbetrachtungen auf den Weg gebracht werden. Auch wurde geplant, Patientenbefragungen einzuführen und eine Verbesserung des Aufwand-NutzenVerhältnisses zu erreichen. ${ }^{210}$

Patientenbefragungen zur Qualitätssicherung sind gerade vor dem Hintergrund des Ziels, das Patientenwohl sicherzustellen, von großer Bedeutung. Zu erwägen ist deshalb,

210 Vgl. Institut für angewandte Qualitätsförderung und Forschung im Gesundheitswesen 2014, 238-244. 
Patientenbefragungen nicht nur bezogen auf Behandlungserfahrungen und Behandlungsergebnisse durchzuführen, wie sie vom AQUA-Institut derzeit für einzelne Leistungsbereiche vorgeschlagen werden, sondern Befragungen auch bezogen auf die subjektive Lebensqualität der Patienten während der Krankenhausbehandlung, den Behandlungsprozess und gegebenenfalls den unerfüllt gebliebenen Informationsbedarf durchzuführen. ${ }^{211}$ Auch sollten die Pflege und ihre Standards in zukünftigen Qualitätserhebungsinstrumenten nicht nur stärker in den bestehenden Leistungsbereichen Berücksichtigung finden, sondern die Pflege sollte auch als eigenständiger Leistungsbereich operationalisiert werden.

Insbesondere der ärztliche Bereich, jedoch auch die Pflege im Krankenhaus wird zunehmend mit Dokumentationspflichten belastet, deren Umfang und Detailliertheit die ohnehin knappen zeitlichen Ressourcen zusätzlich belastet. Offenbar ist diese Form der Dokumentation vor allem für die Weiterentwicklung des Abrechnungssystems erforderlich. Insofern der Nutzen dieser Dokumentation für den individuellen Patienten fraglich ist und die Dokumentation von Ärzten und Pflegenden eine erhebliche zusätzliche Leistung und Aufmerksamkeit verlangt, die dann der Zuwendung zum Patienten abgeht, stellt sich die Frage nach einer geeigneten Kompensation für diese Leistungserbringung sowie nach der Entwicklung digitaler technischer Lösungen für eine durchgreifende Vereinfachung der Dokumentation.

211 Wie wichtig objektivierbare Erhebungsinstrumente für Befragungen zur Patientenzufriedenheit sind, zeigt die Befragung von AOK und anderen Krankenkassen aus dem Jahr 2012, wonach zwar 83 Prozent der Patienten überwiegend hohe Zufriedenheit mit der Versorgung im Krankenhaus geäußert haben, aber die Repräsentativität der Befragung nicht gegeben und damit ihre Aussagekraft nicht hinreichend ist (Weisse Liste/Barmer GEK/ AOK 2012, 2). 


\section{ZUSAMMENFASSUNG UND EMPFEHLUNGEN}

\subsection{Zusammenfassung}

\section{Kapitel 1: Einleitung und Problemaufriss}

Organisation und Finanzierung der Krankenhausversorgung in Deutschland sind seit Jahrzehnten Themen einer kontroversen politischen Diskussion. Hierzu tragen ständige Herausforderungen bei, wie die allgemeine Kostenentwicklung im Gesundheitswesen, Weiterentwicklungen in der Medizin mit der Notwendigkeit von Investitionen und einer kontinuierlichen Anpassung der medizinischen Infrastruktur, veränderte Erwartungshaltungen aufseiten der Patienten, das Interesse an der Teilnahme an einem volkswirtschaftlich attraktiven Wachstumsmarkt und die demografische Entwicklung. Es fällt auf, dass in dieser Diskussion eine Ausrichtung auf das Patientenwohl als maßgebliches normatives Leitprinzip für die Krankenhausversorgung bisher nicht explizit im Vordergrund steht. Mit diesem Befund korrespondieren besorgniserregende Entwicklungen in der Krankenhausmedizin, wie zum Beispiel Mengenausweitungen oder Reduzierungen von Behandlungsleistungen, die Konzentration auf besonders gewinnbringende Behandlungsverfahren zulasten anderer notwendiger Behandlungsangebote sowie mittlerweile problematische Arbeitsbedingungen für das im Krankenhaus tätige Personal.

Mit dem im Jahr 2015 beschlossenen Gesetz zur Stärkung der Versorgung in der gesetzlichen Krankenversicherung (GKV-Versorgungsstärkungsgesetz) und dem Gesetz zur Reform der Strukturen der Krankenhausversorgung (Krankenhausstrukturgesetz), Letzteres mit der Forderung, eine „qualitativ hochwertigen“ und „patientengerechten“ Versorgung zu gewährleisten, nimmt der Deutsche Bundestag den Patienten und sein Wohl wieder verstärkt in den Blick und bindet die zukünftige Ressourcenbemessung an diesen übergeordneten 
Maßstab. Es bleibt indes die Herausforderung, wie vom Gesetz gefordert, die Kriterien für diesen Maßstab näher zu bestimmen und für die Patienten und die Gesellschaft transparent zu machen.

Vor diesem Hintergrund setzt sich der Deutsche Ethikrat in der vorliegenden Stellungnahme mit der Krankenhausversorgung in Deutschland auseinander und stellt dabei das Patientenwohl als normatives Leitprinzip in den Mittelpunkt. Dabei ist sich der Deutsche Ethikrat der systematischen und methodischen Schwierigkeiten bewusst, die mit einer Fokussierung von Überlegungen zur Patientenwohlorientierung auf die Krankenhausversorgung als lediglich einzelnes Segment des höchst komplexen Gesamtsystems der Gesundheitsversorgung in Deutschland verbunden sind. Jedoch lassen die problematischen Entwicklungen in der Krankenhausmedizin in Deutschland solche Überlegungen als geboten erscheinen.

\section{Kapitel 2: Überblick über das Versorgungssystem Krankenhaus in Deutschland}

Bereits ein Überblick über Struktur, Finanzierung und rechtliche Rahmenbedingungen zeigt, dass auch das Segment der stationären Versorgung selbst eine komplizierte Konstruktion darstellt, die bisher keine hinreichende Stabilität entwickelt hat, was sich in der offensichtlichen Notwendigkeit fortlaufender gesetzlicher Nachbesserungen zeigt. Auch wenn durch gesetzgeberische Aktivitäten Verbesserungen erreicht wurden, existiert für die einzelnen Krankenhäuser kaum mittelfristige Planungssicherheit. Zudem sind die normativen Referenzpunkte des bisherigen Systems der stationären Versorgung nicht eindeutig bestimmbar.

\section{Kapitel 3: Patientenwohl als ethischer Maßstab}

Die ethisch begründete Patientenwohlorientierung lässt sich anhand dreier Kriterien operationalisieren: die von der Ermöglichung seiner Selbstbestimmung getragene Sorge für den Patienten, eine gute Behandlungsqualität sowie die gerechte 
Verteilung der für die stationäre Versorgung verfügbaren Ressourcen.

Die selbstbestimmungsermöglichende Sorge hat die Respektierung und Achtung des Patienten als Person mit eigenen Vorstellungen, Wünschen, Interessen, einer eigenen Geschichte und mit eigenen Rechten zum Ausgangspunkt und schließt an das Adhärenz-Konzept und das Modell der partizipativen Entscheidungsfindung in der Arzt-Patient- bzw. PflegendePatient- und Therapeut-Patient-Beziehung an. Demzufolge hat sie eine gelingende Kommunikation zur Voraussetzung, die in Inhalt, Art und bezüglich der Rahmenbedingungen auf den Patienten zugeschnitten sein muss und insbesondere in einem Konzept der informierten und selbstbestimmten Einwilligung des Patienten ihr Ziel findet.

Die Behandlungsqualität umfasst objektive wie subjektive Elemente; die Ersteren beziehen sich auf Möglichkeiten und Maßgaben der medizinischen Wissenschaften, die Letzteren auf die Zufriedenheit der Patienten mit der Behandlung. Qualitätsmessung und Qualitätssicherung orientieren sich an dem Modell der Struktur-, Prozess- und Ergebnisqualität. In einer qualitativ hochwertigen und patientengerechten Behandlung findet die Behandlungsqualität ihren Ausdruck insbesondere auch in der verantwortungsvoll gestellten, auf den individuellen Patienten bezogenen medizinischen Indikation.

Gerechtigkeit im Sinne von gleichem Zugang zu Krankenhausleistungen und ihrer gerechten Verteilung umfasst sowohl das Gebot der statusindifferenten Gleichbehandlung (equality) als auch das Gebot des fairen und jeweils individuell angemessenen Einsatzes von Ressourcen (equity). Aufgrund der gegebenen Knappheit der Ressourcen verweisen beide Gerechtigkeitsforderungen auf ihren effektiven (wirksamen) und effizienten (sparsamen) Einsatz. Die Forderung nach einem ressourcenreflexiven Handeln im Krankenhaus darf allerdings nicht zu einer ökonomischen Überformung ärztlichen wie pflegerischen und therapeutischen Handelns führen. 


\section{Kapitel 4: Gefährdungen des Patientenwohls: Konfliktfelder}

Mit Blick auf die das Patientenwohl bestimmenden drei Kriterien lassen sich in der Krankenhausbehandlung in Deutschland ethisch relevante Konfliktfelder identifizieren und Lösungsansätze benennen. Im Vordergrund von patientenbezogenen Entscheidungen stehen gegenwärtig als Outcome-Parameter vor allem die Effektivität und Effizienz der Behandlung. Die anderen für das Patientenwohl relevanten Kriterien der selbstbestimmungsermöglichenden Sorge und des gleichen Zugangs $\mathrm{zu}$ Behandlungsleistungen treten dadurch faktisch in den Hintergrund.

Die sich hieraus ergebenden Konfliktfelder betreffen insbesondere zunehmend mangelnde Möglichkeiten für eine angemessene interpersonale Kommunikation in der Arzt-Patientund Pflegende-Patient- bzw. Therapeut-Patient-Beziehung einschließlich interkultureller Behandlungssituationen sowie die zunehmende Schwierigkeit für die im Krankenhaus tätigen Berufsgruppen, ihre jeweiligen berufsethischen Pflichten umsetzen zu können. Ebenso erweist es sich als zunehmend schwierig, einen gleichen Zugang zu Krankenhausleistungen und ihre gerechte Verteilung insbesondere für Patientengruppen mit besonderen Bedarfen sicherzustellen, wie dies in jeweils unterschiedlicher Weise Kinder und Jugendliche, Patienten in hohem Lebensalter, mit geriatrietypischen Erkrankungen, mit Demenz, mit Behinderung oder Patienten mit Migrationshintergrund betrifft.

Auf der Grundlage dieser Analyse unterbreitet der Deutsche Ethikrat die folgenden Empfehlungen.

\subsection{Empfehlungen}

\section{Gewährleistung einer besseren Kommunikation}

a) Für eine am Patientenwohl orientierte Kommunikation im Krankenhaus sollten der Gesetzgeber und die Selbstverwaltung sicherstellen, dass der zeitliche und organisatorische 
Aufwand bei den Vorgaben für die Vergütung innerhalb des DRG-Systems entsprechend berücksichtigt wird.

b) Im Rahmen der Qualitätsfokussierung, die das Krankenhausstrukturgesetz fordert, sollten Instrumente entwickelt werden, mit denen die Kommunikation transparent dokumentiert wird. Die Dokumentation sollte sowohl Gespräche mit den Patienten als auch interprofessionelle Patientenvisiten und Fallkonferenzen umfassen. Dabei sollte bei den Dokumentationsrichtlinien auf einen vertretbaren Aufwand geachtet werden.

c) Aus Gründen der Gleichbehandlung und der Sicherstellung einer informierten selbstbestimmten Entscheidung sollte der Gesetzgeber die Kosten für eine notwendige professionelle Übersetzung im Kontext einer interkulturellen Behandlungssituation sowie eine Übersetzung in Gebärdensprache für gehörlose Patienten im fallbasierten $\mathrm{Ab}$ rechnungssystem abbilden.

d) Zur Sicherung der Kommunikationsqualität im Krankenhaus sollte die Kommunikationskompetenz aller im Krankenhaus Beschäftigten systematisch durch regelmäßige Aus-, Fort- und Weiterbildungsangebote gestärkt und fortentwickelt werden. Dabei sollte auch interkulturelle Kompetenz vermittelt werden, die durch Informiertheit über andere Kulturen und Verständnis ihrer Wertvorstellungen gekennzeichnet ist.

e) Die bereits von einigen Ärztekammern in der Praxis eingeführten Deutschkurse und Fachsprachprüfungen für nicht deutschsprachige Ärzte und Pflegende sollten flächendeckend eingeführt und verpflichtend gemacht werden.

\section{Sicherung und Verbesserung der Leitungsqualifikation}

Bei der Besetzung von ärztlichen und pflegerischen Leitungspositionen im Krankenhaus sollten zukünftig nicht nur die jeweilige berufliche Kernkompetenz, sondern auch qualifizierte Kenntnisse in Ökonomie, Ethik, Management und Recht maßgeblich sein. In ähnlicher Weise sollten leitende 
Krankenhausmanager neben ihrer ökonomischen Fachkompetenz auch über grundständige Kenntnisse in Medizin und Pflege verfügen. Hierfür müssen entsprechende Fortbildungsangebote, die auch den Erwerb von Praxiserfahrung beinhalten, entwickelt bzw. weiterentwickelt werden.

\section{Verbesserung der Pflegesituation im Krankenhaus}

a) Das Bundesministerium für Gesundheit sollte für eine nachhaltige Verbesserung der Pflegesituation im Krankenhaus sorgen. So sollten Pflegepersonalschlüssel in Abhängigkeit von Stations- und Bereichsgrößen für Krankenhäuser entwickelt und implementiert werden, die sich an der Anzahl der zu versorgenden Patienten und ihren Erkrankungen bzw. ihrem Pflegebedarf orientieren. Dabei ist das spezifische Aufgabenspektrum des Pflegedienstes, des Ärztlichen Dienstes und anderer therapeutischer Dienste in dem jeweiligen Fachgebiet unter Einbeziehung von Zeiten etwa der Übergabe, interprofessioneller Visiten und Fallkonferenzen zwingend zu berücksichtigen.

b) Zudem sollten Mindestquoten für vollexaminierte Pflegekräfte, differenziert nach Fachabteilungen, festgelegt und transparent gemacht und ihre Einhaltung einer regelmäßigen Überprüfung unterzogen werden. Abweichungen von diesen Vorgaben sollten für Patienten und zuweisende Ärzte transparent gemacht werden.

c) In diesem Zusammenhang und unter Berücksichtigung des derzeitigen Mangels an examinierten Pflegekräften auf dem Arbeitsmarkt sollten neue Qualifizierungsmodelle entwickelt und gefördert werden, mit denen zum Beispiel Arzthelferinnen und Arzthelfer zu Pflegekräften berufsbegleitend weitergebildet werden können.

d) Im Interesse einer Verbesserung der Qualität einer patientenwohlorientierten Pflege sollten Bedingungen gezielt gefördert werden, die eine personale Kontinuität in der Pflege der Patienten so weit wie möglich gewährleisten und Methoden des Stellen-Poolings vermieden werden. 


\section{Verringerung von Fehlanreizen bei der Vergütung durch Fallpauschalen (DRGs)}

Der Deutsche Ethikrat empfiehlt dem Gesetzgeber und der Selbstverwaltung zu einer am Patientenwohl orientierten Umgestaltung des DRG-Systems folgende Maßnahmen:

a) Um Fehlanreize zur vorzeitigen Verlegung oder Entlassung von multimorbiden Patienten mit mehreren bestehenden medizinischen Behandlungserfordernissen $\mathrm{zu}$ minimieren, sollte die Möglichkeit geschaffen werden, die notwendigen Behandlungen ohne zeitliche Verzögerungen oder ökonomisch motivierte Verlegungen als getrennte DRGs abzurechnen. Synergieeffekte sollten zu entsprechenden Abschlägen führen. Alternativ sollte das Zusammenziehen mehrerer einschlägiger DRGs in Form einer auf den individuellen Fall bezogenen Paketlösung oder einer übergeordneten neuen DRG ermöglicht werden.

b) Für Patienten, bei denen die tatsächlich entstandenen Kosten durch Fallgruppen nicht realistisch abgebildet werden können, wie bei bestimmten hochaltrigen Patienten, Patienten mit seltenen Erkrankungen, Patienten mit schweren bzw. von multiresistenten Keimen verursachten Infektionen, aber auch Patienten mit besonderen Betreuungsbedarfen, Patienten mit Unterbringungsbeschluss oder Patienten mit besonderen Verhaltensauffälligkeiten sollten, wie bereits vereinzelt vorhanden, neue Vereinbarungsmöglichkeiten für Zusatzentgelte oder andere zusätzliche Vergütungselemente geschaffen werden. Als Grundlage hierfür kann der bereits geltende $\$ 17 \mathrm{~b}$ Abs. 1 Satz 10 KHG zu den „besonderen Einrichtungen“ herangezogen werden, der auf diese Fallgruppen auszuweiten und an klare Kriterien zur Begrenzung auf überprüfbar begründete Indikationen und Diagnosegruppen zu binden wäre.

c) Ergänzend zu dem Zweitmeinungsverfahren, das im Krankenhausstrukturgesetz bereits für bestimmte, mit zeitlichem Vorlauf planbare Eingriffe (Elektiveingriffe) vorgesehen ist, sollten zur Vermeidung unnötiger Eingriffe und 
Prozeduren Vergütungsmodelle entwickelt und geprüft werden, die nicht nur zur Durchführung einer Maßnahme Anreize bieten, sondern auch zu deren begründeter Unterlassung. Hierfür könnte zum Beispiel für bestimmte Diagnosebereiche eine Prozedur „Beobachtung“ neu eingeführt werden, die eine eigene Entgeltbewertung erhält, um die ärztliche Entscheidung zum beobachtenden Zuwarten von einer ökonomischen Sanktionierung zu entheben. Zu binden wäre die Einlösung einer solchen DRG jeweils an eine besondere medizinische Begründung, eine Beobachtung des Patienten durch wiederholte Untersuchung sowie das ausführliche Gespräch mit dem Patienten.

d) Zur Verhinderung von Behandlungen in dafür nicht ausreichend ausgerüsteten oder qualifizierten Krankenhäusern sollten neben der neuen Mindestmengenregelung in definierten Fallgruppen die einschlägigen DRGs an bestimmte Qualifikationen und Ausstattungsniveaus von Einrichtungen gebunden werden. Ziel dieser Regelung sollte sein, Krankenhäuser in diesen Fällen von der Erbringung solcher Leistungen abzuhalten.

e) Für die Finanzierung der Behandlungskosten in der stationären Psychiatrie und Psychosomatik sollte auf der Grundlage des Eckpunktepapiers des Bundesministeriums für Gesundheit vom Februar 2016 ein Entgeltsystem entwickelt werden, das langfristig eine an die hausspezifischen Bedarfe angepasste, ausreichende Personalausstattung gewährleistet und die Unterschiedlichkeit der Behandlungsverläufe bei gleicher Diagnose und die individuell höchst unterschiedlichen Lebensumstände, die für den Verlauf der Erkrankung und der Therapie bei psychiatrischen und psychosomatischen Patienten eine große Rolle spielen, berücksichtigt. Formen der sektorübergreifenden Versorgung sollten durch Verknüpfungen stationärer und ambulanter Angebote ermöglicht werden. 


\section{Ausbau qualitätssichernder Strukturen}

a) Zur Verbesserung der Behandlungsqualität und Bündelung von Fachwissen sollten krankheitsbildorientierte Organisationsmodelle in deutschen Krankenhäusern in Form einer Etablierung von multidisziplinären Zentren, die es bisher nur in sehr geringem und fachlich begrenztem Umfang gibt, durch ein Programm auf Bundesebene gefördert werden. Neben den bereits gesetzlich ermöglichten Behandlungszentren für Menschen mit Behinderung wäre die Schaffung von Zentren für geriatrische Patienten erforderlich.

b) Die gesetzlich vorgegebenen Dokumentationspflichten sollten vereinfacht werden mit dem Ziel, mehr Zeit für die Patientenversorgung freizusetzen. Hierzu sollten unter anderem Modelle mit digitaler Unterstützung entwickelt und erprobt werden. Gleichzeitig sollten aber auch Erweiterungen in der Dokumentation der Kommunikation (interprofessionelle Kommunikation, Kommunikation mit dem Patienten, Kommunikationsqualität) und der „weichen“ Pflegefaktoren (Gespräch, Zuwendung, Kontakt) entwickelt und erprobt werden. Für die Entwicklung und Prüfung solcher Dokumentationsverfahren sollten entsprechende Finanzmittel zur Verfügung gestellt werden.

c) Der Gesetzgeber sollte Maßnahmen verstärken, die darauf abzielen, eine vereinheitlichte Dokumentation und Qualitätssicherung im stationären und ambulanten Bereich einzuführen, um die Abläufe im Krankenhaus effektiver und effizienter zu gestalten, die Kooperation zwischen beiden Sektoren zu vereinfachen und Doppeluntersuchungen auf solche Fälle zu reduzieren, bei denen eine strenge medizinische Indikation für eine erneute Diagnostik besteht.

d) Der Bundesgesetzgeber sollte des Weiteren prüfen, ob im Rahmen der Krankenhausbedarfsplanung der Länder auch das Vorhandensein eines klinischen Ethikkomitees oder äquivalenter Strukturen bzw. die Absicht, ein solches in der Einrichtung zu etablieren, zur Voraussetzung für die 
Zulassung eines Krankenhauses gemacht werden sollte. Des Weiteren sind Möglichkeiten für eine organisationsethische Beratung zu schaffen, auf die die Mitglieder der Klinikleitung zurückgreifen können. Je nach Gegebenheiten bietet sich ein hauseigenes Klinisches Ethikkomitee oder eine externe Beratung an.

\section{Besondere Patientengruppen}

a) Zur Sicherung der angemessenen Krankenhausversorgung von Kindern und Jugendlichen sollten spezifische DRGs für diese Altersgruppen erarbeitet und implementiert werden. Voraussetzung dafür ist es, schnellstmöglich eine auf rein kindermedizinische Daten gründende statistische $\mathrm{Ba}$ sis zu schaffen, die eine Abbildung in rein kindermedizinische DRGs erlaubt. Bis dahin sollte durch ausreichend hohe Sicherstellungszuschläge eine den tatsächlichen Kosten entsprechende Vergütung gewährleistet werden. Alternativ sollte geprüft werden, die stationäre Kinder- und Jugendmedizin aus dem derzeitigen System der Fallpauschalen herauszunehmen und nach zu verhandelnden tagesgleichen Pflegesätzen zu vergüten.

b) Im Sinne der Inklusionsziele der UN-Behindertenrechtskonvention sind Möglichkeiten für Zusatzentgelte $\mathrm{zu}$ schaffen, die den besonderen Belangen von Menschen mit Behinderung und dem damit verbundenen Mehraufwand gerecht werden und verhindern, dass Krankenhäuser die Behandlung dieser Patienten ablehnen.

c) Zur Verbesserung der Behandlung von Menschen mit Behinderung im Krankenhaus wird empfohlen, das Gesetz zur Regelung des Assistenzpflegebedarfs im Krankenhaus von 2009 so zu erweitern, dass auch Menschen mit Behinderung, die Sachleistungen beziehen oder in einem Heim leben, Zugang zu der Leistung der zusätzlichen Krankenhausassistenz haben.

d) Zur barrierefreien Umgestaltung von dafür geeigneten Krankenhäusern sollte ein Förderprogramm mit 
Bundesmitteln aufgelegt werden. Zudem sollte die Barrierefreiheit in den Rahmenkriterien für die Krankenhausplanung aufgenommen werden.

e) Für die qualitativ hochwertige und patientengerechte Versorgung von Menschen mit Behinderung im Krankenhaus sollten die Rahmenbedingungen für die Krankenhausplanung der Länder vorsehen, ausgewählte regionale Krankenhäuser für die Sicherstellung der Versorgung von Menschen mit Behinderung auszuweisen und personell und materiell entsprechend auszustatten.

f) Für die Gruppe der demenzbetroffenen Patienten, die im Krankenhaus behandelt werden, sollte ein Förderprogramm des Bundes für die Schaffung demenzsensibler Strukturen in allgemeinversorgenden Krankenhäusern aufgelegt werden, mit dem Fort- und Weiterbildung des Personals für den angemessenen Umgang mit Demenzerkrankten sowie organisatorische und bauliche Umgestaltungen ermöglicht werden. Zudem wird für eine adäquate Behandlung von demenzkranken Patienten empfohlen, die Vergütungshöhe für die Behandlung von Patienten mit Demenz im Krankenhaus dem Aufwand angemessen anzupassen.

\section{Systembedingte Rahmenbedingungen}

a) Es sollten transparente Kriterien für eine primär am Patientenwohl im vom Deutschen Ethikrat dargelegten Sinne ausgerichtete Krankenhausplanung entwickelt und geprüft werden, inwieweit die Erfüllung dieser Kriterien als notwendige Voraussetzung für eine Zulassung von Krankenhäusern im Landeskrankenhausplan nach \$ 108 SGB V und, anstelle einer bisher überwiegend praktizierten Fortschreibung, als Bedingung für die Fortführung der Zulassung von Krankenhäusern herangezogen und verbindlich gemacht werden kann.

b) Bund und Länder sollten bundeseinheitliche Standards für die Krankenhausplanung entwickeln und einführen, um 
die derzeit bestehenden Unterschiede und Doppelstrukturen der Krankenhausversorgung in den Bundesländern zu minimieren. Dies schließt gegebenenfalls eine Reduzierung der Anzahl bestehender Krankenhäuser nicht aus. In diesem Zusammenhang sollte unter der Maßgabe des Patientenwohls mit Beteiligung von Patientenvertretern und unter Berücksichtigung der für eine flächendeckende Grundversorgung unabdingbaren Fachrichtungen ein geeignetes Verhältnis von wohnortnaher respektive schnell erreichbarer stationärer Versorgung und spezialisierten Zentren mit erweitertem Behandlungsangebot ermittelt werden.

c) Es wird ferner empfohlen, dass die Schnittstellenproblematik zwischen stationärem und ambulantem Sektor erneut unter dem besonderen Aspekt des Patientenwohls im vom Deutschen Ethikrat dargelegten Sinne im Hinblick auf das Gesundheitswesen im Allgemeinen und das Krankenhaus im Besonderen systematisch analysiert und evaluiert wird und Lösungspfade erarbeitet und implementiert werden.

d) Der Gemeinsame Bundesausschuss sollte in seine Arbeit fachethische Expertise einbeziehen. Dies kann etwa in Form eines mit einschlägigen Experten multidisziplinär besetzten beratenden Ethikgremiums geschehen. 


\section{LITERATURVERZEICHNIS}

Arolt, V.; Driessen, M.; Dilling, H. (1997): The Lübeck General Hospital Study. I: Prevalence of psychiatric disorders in medical and surgical inpatients. In: International Journal of Psychiatry in Clinical Practice, 1 (3), 207-216.

Augurzky, B. et al. (2015): Krankenhaus Rating Report 2015. Bad Bank für Krankenhäuser - Krankenhausausstieg vor der Tür? Heidelberg.

Augurzky, B.; Beivers, A.; Gülker, R. (2012): Bedeutung der Krankenhäuser in privater Trägerschaft. Essen.

Ausserhofer, D. et al. (2014): Prevalence, patterns and predictors of nursing care left undone in European hospitals: results from the multicountry crosssectional RN4CAST study. In: BMJ Quality \& Safety, 23 (2), 126-135.

Aylett, V. (2010): Do geriatricians need guidelines? In: BMJ, 341:C5340. DOI: 10.1136/bmj.c5340.

Barkowski, M. (2008): Mein Blut tut weh. Interkulturelle Implikationen beim Dolmetschen im Krankenhaus. In: MDÜ, 54 (2), 41-43.

Benner, P. (2000): Stufen zur Pflegekompetenz. From Novice to Expert (3. Aufl.). Bern.

Berendonk, C. et al. (2011): Biographiearbeit in der stationären Langzeitpflege von Menschen mit Demenz. Potenziale des DEMIAN-Pflegekonzepts. In: Zeitschrift für Gerontologie und Geriatrie, 44 (1), 13-18.

Bertelsmann Stiftung (Hg.) (2006): Interkulturelle Kompetenz - Schlüsselkompetenz des 21. Jahrhunderts. Thesenpapier der Bertelsmann Stiftung auf Basis der Interkulturellen-Kompetenz-Modelle von Dr. Darla K. Deardorff. Gütersloh.

Berufsgenossenschaft für Gesundheitsdienst und Wohlfahrtspflege $(\mathrm{Hg}$. (2013): Gesundheitssektor Krankenhaus. Zahlen - Daten - Fakten. Hamburg.

Bioethik-Kommission des Landes Rheinland-Pfalz (Hg.) (2010): Gesundheit und Gerechtigkeit. Ethische, soziale und rechtliche Herausforderungen. Mainz.

Borchelt, M. (2004): Gewachsene Strukturen, beschleunigter Wandel und divergierende Interessenlagen zur Geriatrie: Anatomie eines scheinbaren Widerspruchs. http://www.geriatrie-drg.de/Public/Docs/ Geriatriestrukturen_13022004.pdf [22.02.2016].

Bräutigam, H. H.; Kruse, K. (1992): Die Lifestyle-Klinik. In: Zeit Online, 12.06.1992. http://www.zeit.de/1992/25/die-lifestyle-klinik [08.03.2016].

Brock, D. W.; Wikler, D. (2006): Ethical issues in resource allocation, research, and new product development. In: Jamison, D. T. et al. (Hg.): Disease Control Priorities in Developing Countries (2. Aufl.). Washington D. C., 259-270.

Budroni, H. et al. (2006): Behinderte Pflegewelten. In: Pflege aktuell, 60 (3), 122-127.

Buhr, P.; Klinke, S. (2006): Qualitative Folgen der DRG-Einführung für Arbeitsbedingungen und Versorgung im Krankenhaus unter Bedingungen fortgesetzter Budgetierung. Eine vergleichende Auswertung von vier Fallstudien. Berlin.

Bundesärztekammer (Hg.) (2015a): 118. Deutscher Ärztetag. Beschlussprotokoll. http://www.bundesaerztekammer.de/fileadmin/user_upload/downloads/ pdf-Ordner/118._DAET/118DAETBeschlussprotokoll20150515.pdf [06.09.2015]. 
Bundesärztekammer (Hg.) (2015b): Medizinische Indikationsstellung und Ökonomisierung. http://www.bundesaerztekammer.de/fileadmin/ user_upload/downloads/pdf-Ordner/Stellungnahmen/Stn_Medizinische_ Indikationsstellung_und_OEkonomisierung.pdf [22.02.2016].

Bundesministerium für Gesundheit (Hg.) (2016): Eckpunkte zur Weiterentwicklung des Psych-Entgeltsystems. http://www.bmg.bund.de/fileadmin/ dateien/Downloads/P/Psych-Entgeltgesetz/160218_Eckpunkte_Psych-EntgeltG_ II_Version.pdf [02.03.2016].

Cabrera, A. (2003): Oh Manager, Schwöret! In: Handelsblatt Online, 18.07.2003. http://www.handelsblatt.com/unternehmen/management/gastbeitrag-ohmanager-schwoeret/2259928.html [08.03.2016].

Cape, J. (2002): Consultation length, patient-estimated consultation length, and satisfaction with the consultation. In: British Journal of General Practice, 52 (485), 1004-1006.

Conradi, E. (2013): Ethik im Kontext sozialer Arbeit. In: EthikJournal, 1 (1), 1-19.

Daniels, N. (2013): Justice and access to health care. In: Zalta, E. N. (Hg.): The Stanford Encyclopedia of Philosophy (Spring 2013 Edition). http://plato. stanford.edu/archives/spr2013/entries/justice-healthcareaccess [22.02.2016].

Daniels, N. (2008): Just Health. Meeting Health Needs Fairly. Cambridge.

Daniels, N. (1996): Justice and Justification. Reflective Equilibrium in Theory and Practice. Cambridge.

Depenheuer, O. (1986): Staatliche Finanzierung und Planung im Krankenhauswesen. Eine verfassungsrechtliche Studie über die Grenzen sozialstaatlicher Ingerenz gegenüber freigemeinnützigen Krankenhäusern. Berlin.

Deutsche Alzheimer Gesellschaft (Hg.) (2015): Patienten mit einer Demenz im Krankenhaus. Begleitheft zum „Informationsbogen für Patienten mit einer Demenz bei Aufnahme ins Krankenhaus“" (4. Aufl.). Berlin.

Deutsche Alzheimer Gesellschaft (Hg.) (2013): Menschen mit Demenz im Krankenhaus. Auf dem Weg zum demenzsensiblen Krankenhaus. Berlin.

Deutsche Gesellschaft für Erziehungswissenschaft (Hg.) (2010): Ethik-Kodex der Deutschen Gesellschaft für Erziehungswissenschaft. http://www.dgfe.de/ fileadmin/OrdnerRedakteure/Satzung_etc/Ethikkodex_2010.pdf [22.02.2016].

Deutsche Gesellschaft für Psychoanalyse, Psychotherapie, Psychosomatik und Tiefenpsychologie (Hg.) (2013): Ethik-Leitlinien der DGPT. http://www.dgpt.de/ fileadmin/download/DGPT_Ethik_Leitlinien_Stand_20130927.pdf [22.02.2016].

Deutsche Gesellschaft für Psychologie; Berufsverband Deutscher Psychologinnen und Psychologen (Hg.) (2005): Ethische Richtlinien. http://www. bdp-verband.org/bdp/verband/clips/BDP_Ethische_Richtlinien_2005.pdf [22.02.2016].

Deutscher Berufsverband für Soziale Arbeit (Hg.) (2014): Berufsethik des DBSH. Ethik und Werte. Berlin.

Deutscher Bundestag (Hg.) (1984): Beschlußempfehlung und Bericht des Ausschusses für Arbeit und Sozialordnung (11. Ausschuß). Bundestagsdrucksache 10/2565. http://dip21.bundestag.de/dip21/btd/10/025/1002565.pdf [17.02.2016].

Deutscher Bundestag (Hg.) (1969): Bericht der Bundesregierung über die finanzielle Lage der Krankenanstalten in der Bundesrepublik. Bundestagsdrucksache V/4230. http://dipbt.bundestag.de/doc/btd/05/042/0504230.pdf [17.02.2016]. 
Deutscher Bundesverband für Logopädie (Hg.) (1998): Berufsordnung der LogopädInnen. https://www.dbl-ev.de/fileadmin/Inhalte/Publikationen/ ooo9_b_berufsordnung.pdf [22.02.2016].

Deutscher Ethikrat (Hg.) (2013): Die Zukunft der genetischen Diagnostik - von der Forschung in die klinische Anwendung. Berlin.

Deutscher Ethikrat (Hg.) (2012): Demenz und Selbstbestimmung. Berlin.

Deutscher Ethikrat (Hg.) (2011): Nutzen und Kosten im Gesundheitswesen - Zur normativen Funktion ihrer Bewertung. Berlin.

Deutscher Fachverband für Kunst- und Gestaltungstherapie (Hg.) (2000): Ethische Grundsätze für Kunst- \& GestaltungstherapeutInnen im DFKGT. http://www.dfkgt.de/download_doc.cfm?doc_UUID=45DFC7A1304875DA97AAC E32519E4F4B\& [22.02.2016].

Deutscher Hebammenverband (Hg.) (2011): Leitbild des Deutschen Hebammenverbandes (1. Aufl.). Karlsruhe.

Deutscher Verband der Ergotherapeuten (Hg.) (2005): Ethikkodex und Standards zur beruflichen Praxis der Ergotherapie. https://www.dve.info/ fileadmin/upload/pdf/infothek/Ethik.pdf [22.02.2016].

Deutsches Institut für angewandte Pflegeforschung (Hg.) (2014): PflegeThermometer 2014. Eine bundesweite Befragung von leitenden Pflegekräften zur Pflege und Patientenversorgung von Menschen mit Demenz im Krankenhaus. Köln.

Donabedian, A. (1980): Explorations in Quality Assessment and Monitoring. Volume 1: The Definition of Quality and Approaches to Its Assessment. Ann Arbor.

Donabedian, A. (1966): Evaluating the quality of medical care. In: The Milbank Memorial Fund Quarterly, 44 (3, Part 2), 166-203.

Eichhorn, S. (1975): Krankenhausbetriebslehre. Theorie und Praxis des Krankenhausbetriebes, Band I (3. Aufl.). Köln.

Emanuel, E. J.; Emanuel L. L. (1992): Four models of the physician-patient relationship. In: Journal of the American Medical Association, 267 (16), 2221-2226.

Feißt, M.; Molzberger, K. (2016): Die Praxis der Zahlen im Krankenhausmanagement. Fakt oder Fetisch? In: Bode, I.; Vogd, W. (Hg.): Mutationen des Krankenhauses. Soziologische Diagnosen in organisations- und gesellschaftstheoretischer Perspektive. Wiesbaden, 119-142.

Fleischhauer, K. (2015): Die Regulierung der medizinischen Versorgung in Deutschland. Normsetzung und Normen in der gesetzlichen und in der privaten Krankenversicherung - Eine Einführung. Berlin.

Forum selbstbestimmter Assistenz behinderter Menschen (Hg.) (2007): Ich muss ins Krankenhaus ... und nun? Dokumentation der Kampagne 2006/2007. Berlin.

Friesacher, H. (2015): Wider die Abwertung der eigentlichen Pflege. In: Intensiv, 23 (4), 200-214.

Fuchs, C.; Nagel, E.; Raspe, H. (2009): Rationalisierung, Rationierung und Priorisierung - was ist gemeint? In: Deutsches Ärzteblatt, 106 (12), A554-A557.

Füsgen, I. (Hg.) (1996): Der ältere Patient. Problemorientierte Diagnostik und Therapie (2. Aufl.). München. 
Geisler, L. S. (2010): Das ärztliche Gespräch - eine vernachlässigte Aufgabe. In: Bruchhausen, W.; Hofer, H.-G. (Hg.): Ärztliches Ethos im Kontext. Historische, phänomenologische und didaktische Analysen. Bonn, 99-112.

Geissler, A.; Busse, R. (2015): Stationäre Kapazitätssteuerung im internationalen Vergleich. In: Klauber, J. et al. (Hg.): Krankenhaus-Report 2015. Schwerpunkt: Strukturwandel. Stuttgart, 13-22.

Georg, J.; Frowein, M. (1999): Pflegelexikon. Wiesbaden.

GKV-Spitzenverband (Hg.) (2016): Kennzahlen der gesetzlichen Krankenversicherung. https://www.gkv-spitzenverband.de/media/grafiken/ gkv_kennzahlen/kennzahlen_gkv_2015_94/GKV_Kennzahlen_Booklet_ Q4-2015_30odpi_2016-03-10.pdf [23.03.2016].

Gotzmann, M. et al. (2011): Transcatheter aortic valve implantation in patients with severe symptomatic aortic valve stenosis - predictors of mortality and poor treatment response. In: American Heart Journal, 162 (2), 238-245.

Haas, C. et al. (Hg.) (2015): Gutachten zur ambulanten Notfallversorgung im Krankenhaus - Fallkostenkalkulation und Strukturanalyse. http://www.dkgev. de/media/file/19401.2015-02-17_Gutachten_zur_ambulanten_Notfallversorgung_ im_Krankenhaus_2015.pdf [23.02.2016].

Haskard Zolnierek, K. B.; DiMatteo, M. R. (2009): Physician communication and patient adherence to treatment: a meta-analysis. In: Medical Care, 47 (8), 826-834.

Heffels, W. M. (2002): Pflegeethik als Verpflichtung zur Wahrnehmung personaler Verantwortung der Pflegenden in funktionalisierten Handlungsfeldern der Pflege. Dissertation. Gerhard-Mercator-Universität Duisburg, Fakultät für Gesellschaftswissenschaften. https://duepublico.uni-duisburg-essen.de/ servlets/DerivateServlet/Derivate-5219/heffelsdiss.pdf [22.02.2016].

Herbst, U. (2000): Pflegeberufe. In: Korff, W.; Beck, L.; Mikat, P. (Hg.): Lexikon der Bioethik. Band 3: Pe-Z. Gütersloh, 19-22.

Hoefert, H.-W. (2008): Einbindung von Patienten in den Behandlungsprozess. In: Hoefert, H.-W.; Hellmann, W. (Hg.): Kommunikation als Erfolgsfaktor im Krankenhaus. Heidelberg, 151-170.

Höfling, W. (2008): Zukunftsfragen des Krankenhauses im Gesundheitsgewährleistungsstaat - vom Krankenhausrecht zum Krankenhausregulierungsrecht. In: Ministerium für Arbeit, Gesundheit und Soziales des Landes NordrheinWestfalen (Hg.): Das Krankenhaus im Gesundheitsgewährleistungsstaat. Stuttgart, 17-34.

Höfling, W. (2007): Vom Krankenhausrecht zum Krankenhausregulierungsrecht. In: GesundheitsRecht, 6 (7), 289-294.

Huster, S.; Kaltenborn, M. (Hg.) (2010): Krankenhausrecht. Praxishandbuch zum Recht des Krankenhauswesens (1. Aufl.). München.

Ilkilic, I. (2014): Interkulturalität in der Ethikberatung. In: Coors, M.; Grützmann, T.; Peters, T. (Hg.): Interkulturalität und Ethik. Der Umgang mit Fremdheit in Medizin und Pflege. Göttingen, 101-111.

Ilkilic, I. (2008): Kulturelle Aspekte bei ethischen Entscheidungen am Lebensende und interkulturelle Kompetenz. In: Bundesgesundheitsblatt Gesundheitsforschung - Gesundheitsschutz, 51 (8), 857-864.

Ilkilic, I. (2006): Begegnung und Umgang mit muslimischen Patienten. Eine Handreichung für die Gesundheitsberufe (6. Aufl.). Bochum. 
Ilkilic, I. (2002): Der muslimische Patient. Medizinethische Aspekte des muslimischen Krankheitsverständnisses in einer wertpluralen Gesellschaft. Münster.

Institut für angewandte Qualitätsförderung und Forschung im Gesundheitswesen (Hg.) (2014): Qualitätsreport 2013. Göttingen.

Internationaler Hebammenverband (Hg.) (2014): International Code of Ethics for Midwives. http://www.internationalmidwives.org/assets/ uploads/documents/CoreDocuments/CD2008_001\%20V2014\%20ENG\%20 International\%20Code\%200f\%20Ethics\%20for\%20Midwives.pdf [22.02.2016].

Isensee, J.; Kirchhof, P. (Hg.) (1992): Handbuch des Staatsrechts. Band V: Allgemeine Grundrechtslehren. Heidelberg.

Jaggar, A. M. (1995): Caring as a feminist practice of moral reason. In: Held, V. (Hg.): Justice and Care. Essential Readings in Feminist Ethics. Boulder, 179-202.

Klemperer, D. (2003): Wie Ärzte und Patienten Entscheidungen treffen. Konzepte der Arzt-Patient-Kommunikation. Berlin.

Klinkhammer, G.; Krüger-Brand, H. E. (2015): „Mut zum Beziehungsaufbau“. In: Deutsches Ärzteblatt, 112 (21-22), A966-A972.

Krobath, T.; Heller, A. (Hg.) (2010): Ethik organisieren. Handbuch der Organisationsethik. Freiburg.

Lachetta, R. et al. (2011): Erleben von Menschen mit geistiger Behinderung während eines akutstationären Aufenthaltes. Eine systematische Literaturübersicht. In: Pflegewissenschaft, 14 (3), 139-148.

Lahmann, C.; Dinkel, A. (2014): Ärztliche Gesprächsführung - eine hohe Kunst. In: MMW - Fortschritte der Medizin, 156 (Sonderheft 2), 84-87.

Laufs, A.; Kern, B.-R. (Hg.) (2010): Handbuch des Arztrechts (4. Aufl.). München. Lay, R. (2012): Ethik in der Pflege. Ein Lehrbuch für die Aus-, Fort- und Weiterbildung (2. Aufl.). Hannover.

Loh, A. et al. (2007): Patientenbeteiligung bei medizinischen Entscheidungen. Effekte der Partizipativen Entscheidungsfindung aus systematischen Reviews. In: Deutsches Ärzteblatt, 104 (21), A1483-A1488.

Marckmann, G.; Strech, D. (2009): Auswirkungen der DRG-Vergütung auf ärztliche Entscheidungen: Eine ethische Analyse. In: Zeitschrift für medizinische Ethik, 55 (1), 15-27.

Nagel, E. (Hg.) (2013): Das Gesundheitswesen in Deutschland. Struktur, Leistungen, Weiterentwicklung (5. Aufl.). Köln.

Nagel, E. (Hg.) (2012): Die gesundheitliche Versorgung in Deutschland - eine kurze Einführung. Köln.

Nationale Akademie der Wissenschaften Leopoldina; Deutsche Akademie der Technikwissenschaften; Union der deutschen Akademien der Wissenschaften (Hg.) (2015): Zur Gesundheitsversorgung von Asylsuchenden. http://www. leopoldina.org/uploads/tx_leopublication/2015_Gesundheit_Asylsuchende_01. pdf [01.03.2015].

Pinkert, C.; Holle, B. (2012): Menschen mit Demenz im Akutkrankenhaus. Literaturübersicht zu Prävalenz und Einweisungsgründen. In: Zeitschrift für Gerontologie und Geriatrie, 45 (8), 728-734.

Preusker, U. K. (2010): Lexikon des deutschen Gesundheitssystems (3. Aufl.). Heidelberg. 
Quaas, M.; Zuck, R. (Hg.) (2014): Medizinrecht (3. Aufl.). München.

Rabe, M. (2009): Ethik in der Pflegeausbildung. Beiträge zur Theorie und Didaktik. Bern.

Reifferscheid, A.; Pomorin, N.; Wasem, J. (2015): Ausmaß von Rationierung und Überversorgung in der stationären Versorgung. Ergebnisse einer bundesweiten Umfrage in deutschen Krankenhäusern. In: Deutsche Medizinische Wochenschrift, 140 (13), e129-e135.

Reifferscheid, A.; Pomorin, N.; Wasem, J. (2014): Umgang mit Mittelknappheit im Krankenhaus - Rationierung und Überversorgung medizinischer Leistungen im Krankenhaus? Executive Summary. Universität Duisburg-Essen, Lehrstuhl für Medizinmanagement.

Reiter-Theil, S. (2000): Patientenethik. In: Korff, W.; Beck, L.; Mikat, P. (Hg.): Lexikon der Bioethik. Band 2: G-Pa. Gütersloh, 842-844.

Richter-Kuhlmann, E. (2015): Mut haben, etwas nicht zu tun. In: Deutsches Ärzteblatt, 112 (44), A1810-A1811.

Rixen, S. et al. (2003): Zum rechtlichen Schutz der Patientenautonomie in der ressourcenintensiven Hochleistungsmedizin: Vorschläge zur Neustrukturierung des Aufklärungsgesprächs. In: Medizinrecht, 21 (4), 191-194.

Rosenberger, M. et al. (2009): Ethikkodex professioneller Seelsorger. In: Stimmen der Zeit, 227 (7), 447-458.

Roser, J.-M.; Budroni, H.; Schnepp, W. (2011): Abschlussbericht zum Projekt Entwicklung einer Zielvereinbarung zur barrierefreien Krankenhausversorgung von Menschen mit Mehrfachbehinderungen. http://www.barrierefreiheit. de/tl_files/bkb-downloads/Projekte/barrierefreies_krankenhaus_ mehrfachbehinderung/endfassung_abschlussbericht_barrierefreies_ krankenhaus_bvjn_uwh_2011_druckfassung.pdf [22.02.2016].

Sabaté, E. (2001): Adherence to Long-Term Therapies: Policy for Action. http:// www.who.int/chp/knowledge/publications/adherencerep.pdf [07.03.2016].

Sachverständigenrat zur Begutachtung der Entwicklung im Gesundheitswesen (Hg.) (2014): Bedarfsgerechte Versorgung - Perspektiven für ländliche Regionen und ausgewählte Leistungsbereiche. Gutachten 2014. Bern.

Sachverständigenrat zur Begutachtung der Entwicklung im Gesundheitswesen (Hg.) (2012): Wettbewerb an der Schnittstelle zwischen ambulanter und stationärer Gesundheitsversorgung. Sondergutachten 2012. Bern.

Sattar, S. P. et al. (2004): Patient and physician attitudes to using medications with religiously forbidden ingredients. In: Annals of Pharmacotherapy, 38 (11), 1830-1835.

Satzinger, W.; Werner, A. (2005): Gerechtigkeit im Krankenhaus - Mutmaßungen über Maßstäbe aus der Patientenperspektive. In: Krukemeyer, M. G.; Marckmann, G.; Wiesing, U. (Hg.): Krankenhaus und soziale Gerechtigkeit. Stuttgart, 111-158.

Sauerland, D. (2002): Gesundheitspolitik in Deutschland. Reformbedarf und Entwicklungsperspektiven. Gütersloh.

Schaller, B.; Baller, G. (2008): Der Zusammenhang zwischen guter Kommunikation und Qualität. Konsequenzen für eine erfolgreiche Unternehmensstrategie. In: Das Krankenhaus, 100 (2), 140-142. 
Schmidt, C. (2010): Die Situation von Patientinnen und Patienten mit geistiger und mehrfacher Behinderung im Krankenhaus aus Sicht des Krankenhauses. In: Bundesverband evangelische Behindertenhilfe ( $\mathrm{Hg}$.): Patientinnen und Patienten mit geistiger und mehrfacher Behinderung im Krankenhaus Problemlagen und Lösungsperspektiven. Dokumentation des Symposiums am 4. Februar 2010. Berlin, 39-49.

Schoenfelder, T.; Klewer, J.; Kugler, J. (2011): Determinants of patient satisfaction: a study among 39 hospitals in an in-patient setting in Germany. In: International Journal for Quality in Health Care, 23 (5), 503-509.

Schreyögg, J. et al. (2014): Forschungsauftrag zur Mengenentwicklung nach § 17 b Abs. 9 KHG. Endbericht. http://www.g-drg.de/cms/content/ download/4861/38231/version/2/file/Endbericht+zum+Forschungsauftrag+gem .+\%C2\%A7+17b+Abs.+9+KHG+\%28Druck\%29.pdf [22.02.2016].

Schulz, R. J.; Kurtal, H.; Steinhagen-Thiessen, E. (2008): Rehabilitative Versorgung alter Menschen. In: Kuhlmey, A.; Schaeffer, D. (Hg.): Alter, Gesundheit und Krankheit. Bern, 334-351.

Seffner, S.; Oberschelp, K. (2013): Führung kann man lernen. In: Deutsches Ärzteblatt (Ärztestellen), 110 (46), 2-4.

Shain, M.; Roemer, M. I. (1959): Hospital costs relate to the supply of beds. In: Modern Hospital, 92 (4), 71-73.

Sørensen, K. et. al (2012): Health literacy and public health: a systematic review and integration of definitions and models. In: BMC Public Health, 12:80. DOI: 10.1186/1471-2458-12-80.

Statistische Ämter des Bundes und der Länder (Hg.) (2010): Demografischer Wandel in Deutschland. Heft 2: Auswirkungen auf Krankenhausbehandlungen und Pflegebedürftige im Bund und in den Ländern. Wiesbaden.

Statistisches Bundesamt (Hg.) (2015a): Diagnosedaten der Patienten und Patientinnen in Krankenhäusern (einschl. Sterbe- und Stundenfälle) 2014. Fachserie 12 Reihe 6.2.1. Wiesbaden.

Statistisches Bundesamt (Hg.) (2015b): Grunddaten der Krankenhäuser 2014. Fachserie 12 Reihe 6.1.1. Wiesbaden.

Statistisches Bundesamt (Hg.) (2014): Bevölkerung mit Migrationshintergrund Ergebnisse des Mikrozensus 2013. Fachserie 1 Reihe 2.2. Wiesbaden.

Statistisches Bundesamt (Hg.) (2008): Grunddaten der Krankenhäuser 2006. Fachserie 12 Reihe 6.1.1. Wiesbaden.

Stemmer, R. (2011): Zur Situation der Pflege im Krankenhaus. In: Pflege \& Gesellschaft, 16 (4), 293-372.

Tamburino, C. et al. (2011): Incidence and predictors of early and late mortality after transcatheter aortic valve implantation in 663 patients with severe aortic stenosis. In: Circulation, 123 (3), 299-308.

Taupitz, J. (2009): Bindungswirkung von Standards im Gesundheitswesen. In: Möllers, T. M. J. (Hg.): Geltung und Faktizität von Standards. Baden-Baden, 63-106.

Teschauer, W. (2015): Menschen mit Demenz im Krankenhaus. Präsentation beim Berliner Tag der Patientenfürsprecher am 16. März 2015. http:// patientenbeauftragter.de/images/pdf/20150316_Vortrag_Dr_Teschauer.pdf [17.10.2015]. 
Teschauer, W. (2014): Demenz im Akutkrankenhaus - Gute Wege sind bekannt aber kaum beschritten. In: Alzheimer Info, 1/2014, 1-2.

Thomae, H. (2006): Krankenhausplanungsrecht. Köln.

Trauschke, T.; Werner, H.; Gerlinger, T. (2009): Zur Diagnostik und Häufigkeit von demenziellen Erkrankungen. Eine prospektive Untersuchung im Alltag einer geriatrischen Klinik (PAOLA-Studie). In: Zeitschrift für Gerontologie und Geriatrie, 42 (5), 385-390.

Tronto, J. C. (1993): Moral Boundaries. A Political Argument for an Ethic of Care. New York; London.

Tuschen, K. H.; Trefz, U. (2010) Krankenhausentgeltgesetz. Kommentar mit einer umfassenden Einführung in die Vergütung stationärer Krankenhausleistungen (2. Aufl.). Stuttgart.

Vogd, W. (2016): Das Missverstehen des Ökonomischen. Oder vom Sündenfall falsch verstandener Rationalitäten im Krankenhaus. In: Bode, I.; Vogd, W. (Hg.): Mutationen des Krankenhauses. Soziologische Diagnosen in organisations- und gesellschaftstheoretischer Perspektive. Wiesbaden, 281-307.

Vogd, W. (2015): Arranging medical and economical logics. Investigating the influence of economic controlling in an internal medicine department. In: Knudsen, M.; Vogd, W. (Hg.): Systems Theory and the Sociology of Health and Illness. Observing Healthcare. Abingdon; New York, 103-127.

Vogd, W. (2014): Götter in Grau. Über das gestörte Verhältnis zwischen Arzt und Patient. Hamburg.

Vogd, W. (2006): Verändern sich die Handlungsorientierungen von Krankenhausärzten unter den neuen organisatorischen und ökonomischen Rahmenbedingungen? Ergebnisse einer rekonstruktiven Längsschnittstudie. In: Sozialer Sinn, 7 (2), 197-229.

Vorstand der Akademie für Ethik in der Medizin (2010): Standards für Ethikberatung in Einrichtungen des Gesundheitswesens. In: Ethik in der Medizin, 22 (2), 149-153.

Warschke, F.; Lauerer, M.; Nagels, K. (2015): Die stationäre Versorgung in Deutschland im internationalen Vergleich. Bern.

Weisse Liste; Barmer GEK; AOK (Hg.) (2012): Ergebnisse aus der Befragung zum Krankenhausaufenthalt. Stand: Dezember 2012. https://www. aok-gesundheitspartner.de/imperia/md/aokbv/presse/pressemitteilungen/ archiv/2012/umfrageergebnisse_121218.pdf [23.02.2016].

Wiesemann, C.; Lenk, C. (2006): Gesundheitsökonomie und Pädiatrie: Ethische Aspekte. In: Gerber, A.; Lauterbach, K. W. (Hg.): Gesundheitsökonomie und Pädiatrie. Stuttgart, 48-54.

Wolf, J.; Ostermann, A. (2016): Von der Organisation der Pflege zur Pflege der Organisation. Ein neuer Typus der Pflegedienstleitung im Krankenhausmanagement. In: Bode, I.; Vogd, W. (Hg.): Mutationen des Krankenhauses. Soziologische Diagnosen in organisations- und gesellschaftstheoretischer Perspektive. Wiesbaden, 165-183.

Woopen, C. (2009): Der Arzt als Heiler und Manager - Zur erforderlichen Integration des scheinbar Unvereinbaren. In: Katzenmeier, C.; Bergdolt, K. (Hg.): Das Bild des Arztes im 21. Jahrhundert. Berlin; Heidelberg, 181-194.

Wunder, M. (2008): Demenz und Selbstbestimmung. In: Ethik in der Medizin, $20(1), 17-25$. 
Zander, B. et al. (2014): Implizite Rationierung von Pflegeleistungen in deutschen Akutkrankenhäusern - Ergebnisse der internationalen Pflegestudie RN4Cast. In: Das Gesundheitswesen, 76 (11), 727-734.

Zentrale Ethikkommission bei der Bundesärztekammer (2013a): Ärztliches Handeln zwischen Berufsethos und Ökonomisierung. Das Beispiel der Verträge mit leitenden Klinikärztinnen und -ärzten. In: Deutsches Ärzteblatt, 110 (38), A1752-A1756.

Zentrale Ethikkommission bei der Bundesärztekammer (2013b): Versorgung von nicht regulär krankenversicherten Patienten mit Migrationshintergrund. In: Deutsches Ärzteblatt, 110 (18), A899-A903.

Zentrale Ethikkommission bei der Bundesärztekammer (2010): Die neue UN-Konvention für die Rechte von Menschen mit Behinderung als Herausforderung für das ärztliche Handeln und das Gesundheitswesen. In: Deutsches Ärzteblatt, 107 (7), A297-A300.

Zentrale Ethikkommission bei der Bundesärztekammer (2006): Ethikberatung in der klinischen Medizin. In: Deutsches Ärzteblatt, 103 (24), A1703-A1707.

Zuckerman, M. et al. (2002): Determinants of women's choice of obstetrician/ gynecologist. In: Journal of Women's Health \& Gender-Based Medicine, 11 (2), 175-180. 


\section{ABKÜRZUNGEN}

a. F.

Abs.

AQUA-Institut

BAnz.

BGBI.

BVerfGE

DRG

G-BA

GG

GKV

GVBI.

ICD

InEK

IQTIG

IQWiG

KHG

m. w. N.

OECD

OPS

Rn.

SGB V

SGB XI

TAVI

TISS

ZEKO alte Fassung

Absatz

Institut für angewandte Qualitätsförderung und Forschung im Gesundheitswesen

Bundesanzeiger

Bundesgesetzblatt

Entscheidungen des Bundesverfassungsgerichts

Diagnosis Related Group (diagnosebezogene

Fallpauschale)

Gemeinsamer Bundesausschuss

Grundgesetz

Gesetzliche Krankenversicherung

Gesetz- und Verordnungsblatt

Internationale statistische Klassifikation der Krankheiten und verwandter Gesundheitsprobleme

Institut für das Entgeltsystem im Krankenhaus

Institut für Qualitätssicherung und Transparenz im Gesundheitswesen

Institut für Qualität und Wirtschaftlichkeit im Gesundheitswesen

Gesetz zur wirtschaftlichen Sicherung der Krankenhäuser und zur Regelung der Krankenhauspflegesätze

(Krankenhausfinanzierungsgesetz)

mit weiteren Nachweisen

Organisation für wirtschaftliche Zusammenarbeit und Entwicklung

Operationen- und Prozedurenschlüssel

Randnummer

Fünftes Buch Sozialgesetzbuch

Elfes Buch Sozialgesetzbuch

Transcatheter Aortic Valve Implantation

Therapeutic Intervention Scoring System

Zentrale Ethikkommission bei der Bundesärztekammer 




\section{Mitglieder des Deutschen Ethikrates}

Prof. Dr. med. Christiane Woopen (Vorsitzende)

Wolf-Michael Catenhusen, Staatssekretär a. D. (Stellvertretender Vorsitzender)

Prof. Dr. theol. Peter Dabrock (Stellvertretender Vorsitzender)

Prof. Dr. iur. Jochen Taupitz (Stellvertretender Vorsitzender)

Prof. Dr. med. Katrin Amunts

Constanze Angerer, Präsidentin a. D. des Landgerichts München I

Prof. Dr. med. Frank Emmrich

Dr. med. Christiane Fischer

Prof. Dr. phil. habil. Dr. phil. h. c. lic. phil. Carl Friedrich Gethmann

Bischof Prof. Dr. theol. Martin Hein

Prof. Dr. med. Dr. phil. Thomas Heinemann

Prof. Dr. iur. Wolfram Höfling

Prof. Dr. (TR) Dr. phil. et med. habil. Ilhan Ilkilic, M. A.

Prof. Dr. med. Leo Latasch

Weihbischof Dr. theol. Dr. rer. pol. Anton Losinger

Prof. Dr. iur. Reinhard Merkel

Herbert Mertin, Justizminister a. D. des Landes Rheinland-Pfalz

Prof. Dr. med. habil. Dr. phil. Dr. theol. h. c. Eckhard Nagel

Dr. phil. Peter Radtke

Ulrike Riedel, Rechtsanwältin, Staatssekretärin a. D.

Prof. em. Dr. iur. Edzard Schmidt-Jortzig, Bundesminister a. D.

Prof. Dr. theol. Eberhard Schockenhoff

Prof. Dr. med. Elisabeth Steinhagen-Thiessen

Prof. Dr. iur. Silja Vöneky

Prof. Dr. med. Claudia Wiesemann

Dipl.-Psych. Dr. phil. Michael Wunder

\section{Mitarbeiterinnen und Mitarbeiter der Geschäftsstelle}

Dr. rer. nat. Joachim Vetter (Leiter)

Dr. theol. Katrin Bentele

Carola Böhm

Ulrike Florian

Steffen Hering

Christian Hinke

Petra Hohmann

Christian M. Jolibois

Torsten Kulick

Dr. Nora Schultz 